Central Files Number

57-11-3

Revision 1

C-86 - Nuclear Rocket Engines

\title{
AN ANALYSIS OF VORTEX TUBES FOR \\ COMBINED GAS-PHASE FISSION- \\ HEATING AND SEPARATION OF \\ THE FISSIONABLE MATERIAL
}

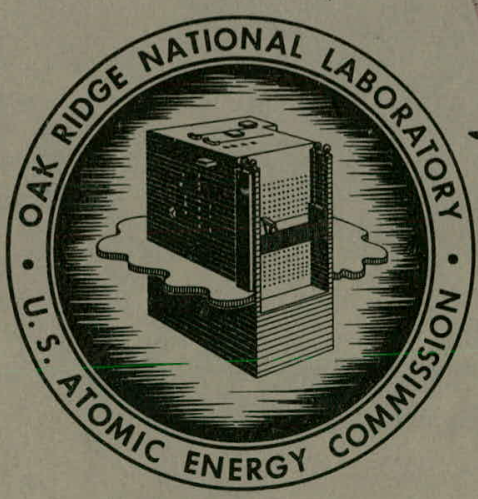

NOTICE

This document contains information of a preliminary nature and was prepared primarily for internal use at the Oak Ridge National Laboratory. It is subiect to revision or correction and therefore does not represent a final report.

OAK RIDGE NATIONAL LABORATORY

operated by

UNION CARBIDE CORPORATION

for the

U.S. ATOMIC ENERGY COMMISSION

\section{RESTRICTED BATA}

This document contains Restriceded 0 ata as defined in the Atomic Energy Act of 1954. Its ferphnger or the disclosure of its contents in any manner to an ynowhorised person is prohibited. 


\section{DISCLAIMER}

This report was prepared as an account of work sponsored by an agency of the United States Government. Neither the United States Government nor any agency Thereof, nor any of their employees, makes any warranty, express or implied, or assumes any legal liability or responsibility for the accuracy, completeness, or usefulness of any information, apparatus, product, or process disclosed, or represents that its use would not infringe privately owned rights. Reference herein to any specific commercial product, process, or service by trade name, trademark, manufacturer, or otherwise does not necessarily constitute or imply its endorsement, recommendation, or favoring by the United States Government or any agency thereof. The views and opinions of authors expressed herein do not necessarily state or reflect those of the United States Government or any agency thereof. 


\section{DISCLAIMER}

Portions of this document may be illegible in electronic image products. Images are produced from the best available original document. 


\section{JNCLASSIFIED}

\section{LEGAL NOTICE}

Thrs report was prepared ne an account of Goverament sponsored work. Neither the Untted States, nor the Commission, nor any person acting on bohalf of the Commiselon:

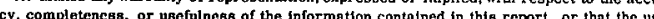
of any informatson, apparatue, method, or procese disclosed in this report may not infringe

privately owned righte; or
B. Assumes any Hnbilities with reapect to the uso of, or for damages resulting from the of any information, npparatus, method, or process disclosed in thes report

As used in the above, "person acting on behalf of the Commlesion" includes any employee or conirnctor of the commission, or employee of such contractor, to the extent that Whe with the Commission, or his employment with such contractor.
C-86 NUCLEAR ROCKET ENGINES

External Transmittal Authorized $\leftarrow$ Distribution.Iimited to

Recipients Indicated

Central Files Number

$$
\text { 57-11-3 }
$$

Revision 1

This document consists of 107pages.

An Analysis of Vortex Tubes for

Combined Gas-Phase Fission-Heating and

Separation of the Fissionable Material

By

J. I. Kerrebrock

R. V. Meghreblian

April 11, 1958

VIVLLASSIFIED

Classification cancelled for changed to

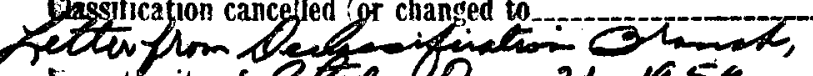

by authrity of Rete on $24-1954$

73

TiE, date $2 \cdot 4-60$

\section{RESTRTCWET DATA}

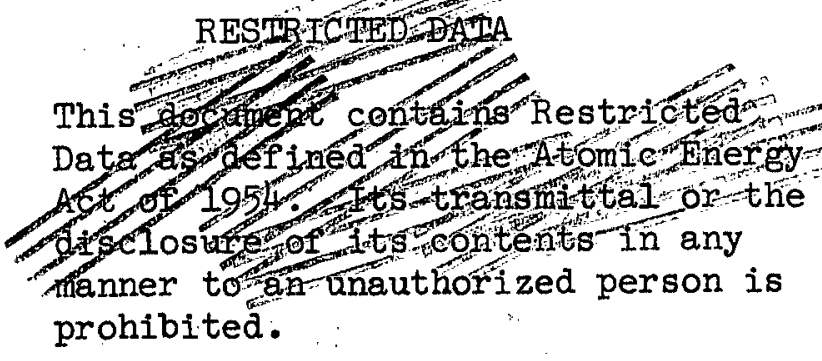

200

mas 
Internal Distribution

1. F. F. Blankenship
2. E. P. Blizard
3: R. A. Charpie
4: W. K. Ergen
5. A. P. Fraas
6. J. H. Frye
7. B. I. Greenstreet
8. W. R. Grimes
9: H. W. Hoffman
10.
W. H. Jordan

I. F. F. Blankenship
11. J. L. Kerrebrock

12. J. J. Keyes

13. P. G. Lafyatis

14. W. D. Manly

15-17. R. V. Meghreblian

18. R. F. Newton

19. J. A. Swartout

20. A: M. Weinberg

21. E. P. Wigner

External Distribution

- 22. A. H. Flagg, Aerojet-General Corp.

- 23: AFPR, Douglas, Long Beach

24. AFPR, North American Canoga Park

-25. AFPR, North American Downey

- 26\% Air Research and Development Command (RDGN)

- 27. Air Research and Development Command (RDZPSP)

.28. Air Technical Intelligence Center

- 29. ANP Project Office, Convair, Fort Worth

-30. Albuquerque Operations office

-31. Armed Forces Special Weapons Project, Sandia

- 32. Armed Forces Special Weapons Project, Washington

-33. Army Bailistic Missile Agency

-34. Assistant Secretary of Defense, Rand (WSEG)

- 35-38. Atomic Energy Commission, Washington

-39. Bureau of Aeronautics

- 40. C. D. Pengelley, BAR, Glenn L. Martin, Baltimore

41. Burea of Ordnance

.42. Bureau of Ordnance (SP-209)

-43. Bureau of Ships

- 44. General Electric Company (ANPD)

45. Headquarters, Air Force Special Weapons Center

46. J. B. Robinson, III, Headquarters, USAF, Washington

- 47. F. Goddard, Jet Propulsion Laboratory

. 48. H. J. Stewart, Jet Propulsion Laboratar

- 49. P. Wegener, Jet Propuls som Laforator

-50-51. J. Todd, Los Alamoshs

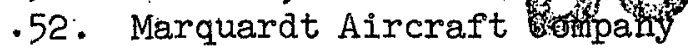

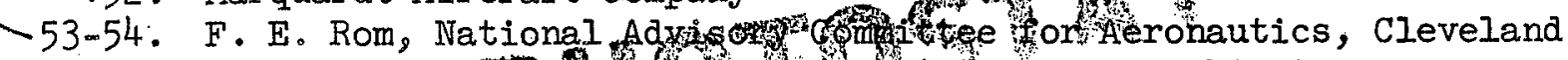

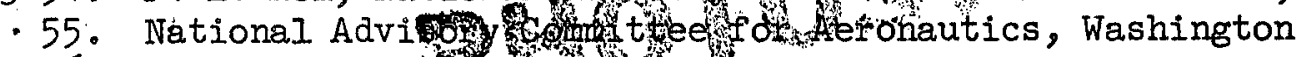

56. Office of Navol Rethen

57. Office of the Chief of Naval Operations (Op-361)

- 58. Office of the Chief of Ordnance

59. Patent Braich, Washington

60. Redstone Arsenal

61. San Francisco Operations Office

62-63. G. Safanov, USAF Project RAND

64. U.S. Naval Ordnance Test Station

65-66. University of California Radiation Laboratory, Livermore

67-68. Western Development Divisicn

69-74. Wright Air Development Center (WCOSI-3)

75-109. Technical Information Servíce Extension, Oak Ridge 
In order to achieve the high exhaust gas temperatures, which are desirable if the fuil potential of nuclear fission as an energy source for rocket propulsion is to be realized, it seems essential that the fissionable material be maintained in a gaseous mixture with the prow pellant. It is then necessary to separate the fissionable material from the propellant before discharging the latter, since the loss of fissionable material is prohibitive otherwise.

This report presents an analytical evaluation of the characteristics of a vortex tube which achieves the desired separation by means of a centrifugal field. Propellant is fed into the tube tangentially, at the periphery, and diffuses radially inward through a cloud of fissionable gas, picking up the fission heat as it goes. The fissionable gas is held against this radial propellant flow by the centrifugal vortex field generated by the tangentially entering propellant.)

The analysis involves several assumptions, the most important of which are that the flow is laminar and that it is inviscid. A set of non-linear first order differential equations is obtained which is sufficient to describe the fissionable gas concentration, temperature, and pressure distributions in the tube. These equations have been integrated numerically for a very wide range of conditions.

The analysis predicts that the vortex tube is capable of maintaining rather high concentrations of fissionable gas, such that the density of the fissionable gas is of the same order as that of the propellant, with negligible loss of the fissionable gas, and with ratios of propellant exit temperature to entrance temperature up to ten. The permissible propellant mass flow is found to be dependent principaliy on the entering tangential Mach number of the propellant. The permissible mass flow per unit of tube length is independent of the tube diameter, thus a large number of small tubes, filling a given volume, have a much larger mass flow capacity than a single tube of the same volume. For an inlet tangential Mach number of unity, the permissible mass flow is of the order of 0.01 pounds per second per foot of tube length. 
A set of experiments, designed to verify the most important assumptions of the analysis, is suggested. The first of these is a room temperature experiment intended to verify that vortex strengths approaching those implied by the analysis can actually be obtained with the low mass flow rates which are permitted by the diffusion process. Contingent upon the success of this first experiment, a second experiment using a mixture of hydrogen and some heavy gas such as mercury vapor or iodine is suggested for verification of the actual separation process.

A discussion of the performance characteristics of the vortex tube as a rocket propulsion device is also presented. Some numerical examples are given to indicate the order of magnitude of the various interesting parameters; however, these should not form a basis for judgement of the performance, since no attempt at optimization has been made. More critical studies of the nuclear and performance aspects of the device are being initiated at ORNL, and will be the subject of a later report. 


\section{Table of Contents}

Page

Introduction

Analysis of the Heating-Separation Process

Nomenclature

Mathematical formulation

Momentum equations

Energy equation

Conservation equation for the heavy gas

Diffusion equation

24

Diffusion coefficient

Solution for the derivatives of $n_{2}^{*}$ and p. $^{*}$

Maxima and Minima of $\mathrm{w}$

Method of integration

Parameters of the heating separation process

Results

40

The density ratio, dimensionless temperature, and dimensionless fuel concentration

40

Dependence of the performance parameters on the choice of $r_{p}^{*} 41$

Dependence of the nuclear-coupling parameters on the choice of $r_{p}^{*}$

The overail temperature ratio

The overall pressure ratio 
The peripheral Mach number

44

The relative mass $-f$ low capacity 45

The exit to peripheral radius ratio 45

Nuclear coupling parameters $\quad 46$

Discussion of the Principle Assumptions and

Proposal for Experimental Verification

47

stability of the flow in the vortex tube 47

Estimate of viscous effects 48

Suggestions for an experimental verification of the analysis 52

Performance and Criticality Estimates $\quad 54$

Heat balance 54

Criticality 55

Performance $\quad 56$

Numerical examples : : $\quad 59$

References 62

List of Figures and Tables 63 
Introduction

The performance of a rocket engine is for most purposes characterized by its thrust per unit mass flow rate, or specific impulse. Although it depends somewhat on the pressure-expansion ratio and details of the rocket motor, the specific impulse is principally dependent on the stagnation temperature of the exhaust gas and its molecular weight. It is proportional to the square root of the ratio of the temperature to the molecular weight.

The potential advantages of nuclear fission as an energy source for rocket propulsion are thus two. First, since the propellant is not required to react chemically, it may be chosen to have a low molecular weight. Second, since the energy release in a nuclear reaction occurs at an extremely high energy level, the stagnation temperature of the exhaust gases may at least in principle be very high.

The advantages of low molecular weight may be realized by choosing hydrogen, or some readily dissociated substance such as methane or amonila, as the propellant. However, in order to take advantage of the high potential stagnation temperatures it seems essential that the fissioning material be mixed with the propellant in the gaseous state, so that the bulk of the fission energy is transferred directly from the fission fragments to the gaseous propellant.

It is readily demonstrated by simple order-of-magnitude calculations that in order to add large amounts of energy in this way it is necessary to have rather high concentrations of fissionable material in the gas. If it is then assumed that the mixture of propellant and fissionable material is exhausted, the loss of fissionable material is so high as to be prohibitive. It is therefore necessary to separate the fissionable material from the hot exhaust gas before discharging it.

The purpose of the present report is to describe a method of achieving the necessary high concentrations of fissionable material in the gas, and the necessary separation of the fissionable material. It consists essentially of a vortex tube, in which the fissionable material is held by the centrifugal field while the propellant gas diffuses through it, picking up the fission heat as it moves inward. The propellant gas, and enough fissionable material to make up losses, enter the tube tangentially at the periphery, as shown in Fig. 1 . The propellant then passes spirally imward, through the fissionable material, and leaves the tube near its center, through a convergent-divergent nozzle at one end. The heated gas does not come in contact with the walls, except in the nozzle, so that the process seems superior to one in which a more or less homogeneous fissionable-material-propellant mixture is passed through a critical system, then through a separation device.

\subsection{6}
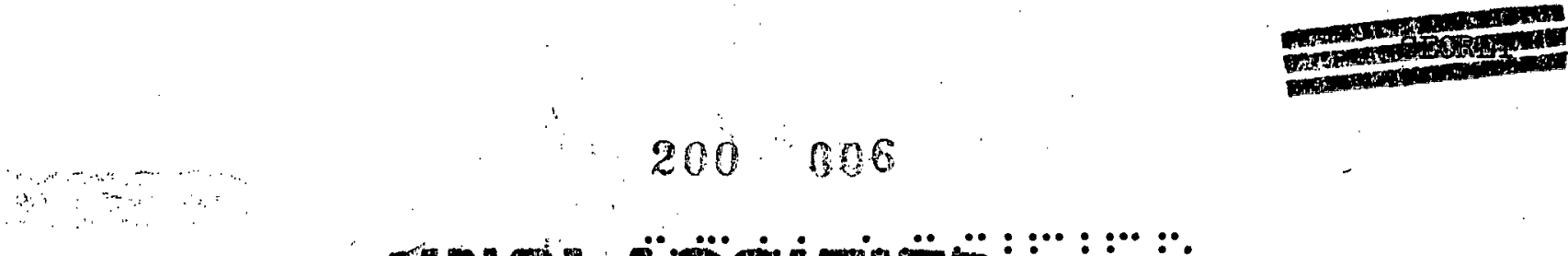

ONCLASSEIFIEOU:I: 


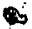

The bulk of the present study consists of a theoretical analysis of the separation-heating process, based on an idealized model which is a first approximation to the physical situation. The principal objectives are to discover the most important parameters governing the process; and establish their ranges; therefore the principal results will take the form of relationships between these parameters.

In order to help give a feeling for the significance of these results, the performance of the vortex tube as a rocket propulsion device will be discussed, and some numerical examples given. It should be emphasized, however, that these examples are in no sense optimum, and should not serve as a basis for judgment of the performance of the system. This judgment can be made only on the basis of thorough nuclear and systems studies. Thus the present study may be regarded as a rather complete evaluation of the fluid mechanical characteristics of the heating-separation device, while the nuclear and systems analyses are later steps in an overall evaluation of its performance.

From another point of view, the study may be regarded as an investigation of a fluid-mechanics problem which is interesting in-itself, quite apart from its applications to rocket propulsion. 
Analysis of the Heating-Separation Process

The flow in the vortex tube is compressible, with strong diffusion and high rates of heat addition. It is in general a three-dimensional flow process, and may be turbulent. Thus, in order to make any progress in analyzing it, some assumptions must be made. The following have been taken as a workable set.

1. The flow is assumed to be two-dimensional, with complete uniformity along the axis of the tube. To make this assumption compatible with the flow of propellant through the system, the propellant is considered to be withdrawn from the tube at some radius, $r$, at which the fissionable material concentration is low enough to make the losses reasonable. The propellant is assumed to enter uniformly at the periphery, $r_{p}$, although it would in fact have to be introduced in jets, as in ig. 1 .

2. The flow is assumed laminar. This assumption is quite possibly in error; however, it is felt to be necessary at the present time. The validity of this assumption and its importance probably can be evaluated only experimentally, although some arguments will be given in its defense.

3. The flow is assumed inviscid. Again this is felt to be necessary at present. An estimate of the validity of the assumption will be given for laminar flow.

4. It is assumed that fission fragment heating of the gas is local, i.e., that the range of fission fragments in the gas is small compared to. characteristic. lengths for the system. This assumption seems to be of marginal validity, but is probably not critical.

5. It is assumed that the molecular concentration of fissionable material is much less than that of the propellant. This is true for all systems considered. 
Nomenclature:

The principal notation used in the analysis is as follows:

$c_{p l}=$ specific heat at constant pressure of light gas

$c_{\text {p2 }} \equiv$ specific heat at constant pressure of heavy gas

$d_{12} \equiv$ effective hard-sphere diameter for collisions between light and heavy gas molecules

$D_{12}$ = binary diffusion coefficient

$D \equiv$ dimensionless diffusion parameter, defined by Eq (23)

g $\equiv$ dimensionless heat generation parameter, defined by Eq (II)

G $\equiv$ energy added per unit volume and per unit time by fission in gas mixture

$\mathrm{H}_{\mathrm{O}} \equiv$ enthalpy of gas mixture

$k \equiv$ Bóltzmann's constant

$m_{1} \equiv$ mass of light gas molecule

$\mathrm{m}_{2}=$ mass of heavy gas molecule

$M_{t} \equiv$ tangential Mach number, based on speed of sound in light gas

$M_{r}$ radial Mach number, based on speed of sound in light gas

OMI $\equiv$ mass flow of propellant per unit length of vortex tube 


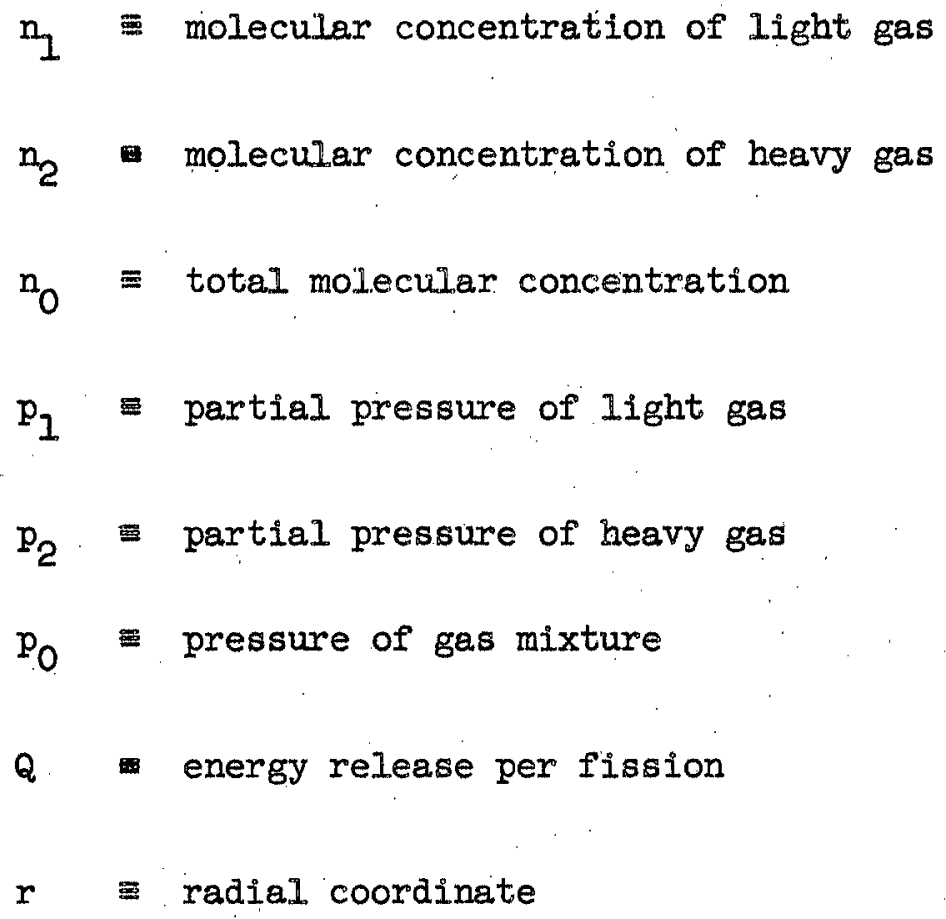


$\gamma \equiv$ ratio of specific heats for light gas

$\lambda \equiv$ coefficient of heat conduction for gas mixture

$P_{1} \equiv$ density of light gas

$P_{2} \equiv$ density of heavy gas

$P_{0} \equiv$ density of gas mixture

$\varnothing \equiv$ neutron track length (neutrons/area/time)

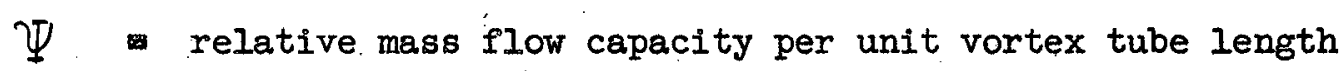

\section{Subscripts:}

On independent variable ( $r$ or $r^{*}$ )

$c$ - exit from tube

$p$ "- periphery of tube

$m$ - point of maximum $w$

On dependent variables

0 - value for gas mixture.

I - value for light gas

2 - value for heavy gas

\section{Superscripts:}

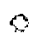

* - quantity divided by its value at point of maximum w. 


\section{Mathematical formulation:}

With the assumption of zero viscosity, the equations governing a binary gas mixture may be written as follows:

Conservation of specie,

$$
u_{0} \frac{d n_{i}}{d r}+\frac{n_{i}}{r} \frac{d\left(u_{0} r\right)}{d r}+\frac{1}{r} \frac{d}{d r}\left(r n_{i} u_{i}\right)=0 \quad, \quad i=1,2
$$

Conservation of tangential momentum,

$$
v_{0} r=\text { constant }
$$

Conservation of radial momentum,

$$
u_{0} \frac{d u_{0}}{d r}-\frac{v_{0}^{2}}{r}=-\frac{1}{\rho_{0}} \frac{d p_{0}}{d r}
$$

Conservation of energy,

$$
\rho_{0} u_{0} r \frac{d H_{0}}{d r}-u_{0} r \frac{d p_{0}}{d r}=r G-\frac{d}{d r}(r q)
$$

where

$$
q \equiv-\lambda \frac{d T}{d r}+T\left(m_{1} c_{p 1} n_{1} u_{1}+m_{2} c_{p 2} n_{2} u_{2}\right)+k K_{T} n_{0}\left(u_{1}-u_{2}\right) T
$$

Here $K_{r}$ is the thermal diffusion ratio. This term is negligible and will be dropped. 
Diffusion equation,

$$
u_{1}-u_{2}=-\frac{n_{0}^{2}}{n_{1} n_{2}} D_{12}\left\{\frac{d}{d r}\left(\frac{n_{1}}{n_{0}}\right)+\frac{n_{1} n_{2}\left(m_{2}-m_{1}\right)}{n_{0} \rho_{0}} \frac{1}{p_{0}} \frac{d p_{0}}{d r}+K_{T} \frac{1}{T} \frac{d T}{d r}\right\}
$$

Again the term involving $\mathrm{K}_{T}$ is negligible and will be dropped.

Equation of state,

$$
p_{i}=n_{i} k T . \quad i=0,1,2
$$

The momentum, energy, continuity, and diffusion equations will now be considered in turn and put in a form suitable for integration.

Momentum equations':

A reference radius, $r_{m}$, will be defined for future convenience as that at which $w$, the density ratio, has its maximum value. Then using the subscript $m$ to denote values at this radius, $\mathrm{Eq}(2)$ may be written, $v_{0}=v_{0_{m}}{ }^{r}$ ? and
Eq (3) becomes,

$$
u_{0} \frac{d u_{0}}{d r}-\frac{\left(v_{0, m^{r} m}\right)^{2}}{r^{3}}=-\frac{1}{\rho_{0}} \frac{d p_{0}}{d r}
$$

Multiplying $\mathrm{Eq}(1)$ by $\mathrm{m}_{i}$ and adding the equations for $i=1$ and $i=2$, we get by using the fact that $m_{1} n_{1} u_{1}+m_{2} n_{2} u_{2}=0$, the continuity equation for the mixture, namely

$$
u_{0} \frac{d \rho_{0}}{d r}+\frac{\rho_{0}}{r} \cdot \frac{d}{d r}\left(r u_{0}\right)=0
$$

or

$$
\frac{d u_{0}}{u_{0}}+\frac{d \rho_{0}}{\rho_{0}}+\frac{d r}{r}=0
$$


which has the solution,

$$
\rho_{0} u_{0}^{r}=\rho_{O m}^{u_{0 m}^{r} m}=\text { constant }
$$

Using Eq (8) to eliminate du $/$ dr from Eq (7), we get after some manipulation,

$$
\frac{d p_{0}}{d r}=\left(\frac{r}{r}\right)^{2}\left\{\left(\frac{\rho_{0 m}}{\rho_{0}}\right) \frac{\rho_{0 m} u^{2}}{r}\left[1+\frac{r}{\rho_{0}} \frac{d \rho_{0}}{d r}\right]+v_{O m}^{2} \frac{\rho_{0}}{r}\right\}
$$

Now

$$
\rho_{0}=n_{1} m_{1}+n_{2} m_{2}=\rho_{1}(1+w)=\frac{m_{1}}{k}(1+w) \frac{p_{1}}{T} \approx \frac{m_{1}}{k}(1+w) \frac{p_{0}}{T}
$$

Hence,

$$
\frac{r}{p_{0}} \frac{d p_{0}}{d r}=\left(\frac{r_{m}}{r}\right)^{2}\left\{\left(\frac{\rho_{0 m}}{\rho_{0}}\right)^{2} \gamma \mathrm{m}_{r m}^{2} \frac{T_{m}}{T}(I+w)\left[1+\frac{r}{\rho_{0}} \frac{d \rho_{0}}{d r}\right]+\gamma m_{t m}^{2} \frac{T_{m}}{T}(I+w)\right\}
$$

where $M_{m}$ and $M_{t m}$ are the radial and tangential Mach numbers at $r_{m}$, based on $\mathrm{u}_{\mathrm{Om}}$ and $\mathrm{v}_{\mathrm{Om}}$ and the speed of sound in the light gas; $\gamma$ is the ratio of specific heats for the light gas. Finally, the equation may be written as,

$$
\frac{r}{p_{0}} \frac{d p_{0}}{d r}=r M_{t m}^{2}(I+w)\left(\frac{r_{m}}{r}\right)^{2} \frac{T_{m}}{T} \cdot\left\{I+\left[I+\frac{r}{\rho_{0}} \frac{d \rho_{0}}{d r}\right]\left(\frac{\rho_{0 m}}{\rho_{0}}\right)^{2}\left(\frac{M_{r m}}{M_{t m}}\right)^{2}\right\}
$$

It will appear later that $\mathrm{M}_{\mathrm{rm}} / \mathrm{M}_{\mathrm{tm}}<10^{-3}$, and the last term in the braces is therefore quite small. 
Denote by an asterisk any quantity divided by its value at $r_{\mathrm{m}}$, eg, $r^{*}=r / r_{m}$ i Neglecting the small term, Eq (9) then becomes;

$$
\frac{r^{*}}{p_{0}^{*}} \frac{d p_{0}^{*}}{d r^{*}}=\gamma M_{t m}^{2} \frac{I+w}{r^{*} 2 T^{*}}
$$

Energy equation:

The factor G in Eq (4) represents the fission heat source. It may be written as

$$
G_{1}=n_{2} \sigma_{f} \phi Q
$$

where $\sigma_{f}$ is the fission cross section for the heavy gas, $\phi$ is the neutron (flux) track length, and $Q$ is the energy release per fission. It will be assumed that these quantities are independent of $x$. The enthalpy, ${ }_{0}$, may be written as,

$$
\mathrm{H}_{0}=c_{p I} \mathrm{~T}\left[\frac{1+w c_{p 2} / c_{p l}}{1+w}\right] \text {, }
$$

but since the molar specific heats of the two gases are of the same order of magnitude,

$$
\text { w. } c_{p 2} / c_{p 1} \approx n_{2} / n_{1} \ll I
$$

hence,

$$
\mathrm{H}_{0} \simeq \frac{\mathrm{c}_{\mathrm{pl}}{ }^{\mathrm{T}}}{1+\mathrm{w}}
$$


If $c_{p l}$ is assumed constant, Eq (4) may then be written in terms of the tem perature as follows:

$$
\begin{aligned}
& \frac{d}{d r}\left[\frac{T}{1+w}\right]=\frac{1}{\rho_{0} c_{p l}} \frac{d p_{0}}{d r}+\frac{n_{2} \sigma_{f} \phi Q}{\rho_{0} u_{0} c_{p l}}- \\
& -\frac{d}{d r}\left[-\frac{\lambda}{\rho_{0} u_{0} c 1} \frac{d T}{d r}+\frac{T}{\rho_{0} u_{0} c_{p l}}\left(m_{1} c_{p 1} n_{1} u_{1}+m_{2} c_{p 2} n_{2} u_{2}\right)\right],
\end{aligned}
$$

the term in $K_{T}$ belng neglected. Again referring all quantities to $r=r_{m}$, this becomes,

$$
\begin{aligned}
& \frac{d}{d r^{*}}\left[\frac{T^{*}}{I+w}\right]=\frac{\gamma-1}{\gamma} \frac{T^{*}}{r^{*}} \frac{I}{1+w} \frac{r^{*}}{p_{0}^{*}} \frac{d p_{0}^{*}}{d r^{*}}+g_{m} n_{2}^{*} r^{*}- \\
& -\frac{d}{d r^{*}}\left\{-\frac{\lambda}{\rho_{O m^{u} m^{r} m^{c} p l}} r^{*} \frac{d^{*}}{d r^{*}}+r^{*} T^{*} \frac{\rho_{2 m^{u} m_{m}}}{\rho_{O m^{u} m^{u}}} n_{2}^{*} u_{2}^{*}\left(\frac{c_{2}}{c_{p l}}-1\right)\right\}
\end{aligned}
$$

where*

$$
g_{m}=-\frac{\sigma_{f} \phi Q n_{2 m}{ }^{r}}{c_{p l} \rho_{0 m}{ }^{u}{ }^{T} m}
$$

*The negative sign in the definition of $\mathrm{g}_{\mathrm{m}}$ is due to the definition of velocities as positive if directed outward. Thus, $u_{0 m}$ is inherently negative. 
Now according to Euchen's formula, (2)

$$
\lambda=\frac{1}{4}(9 r-5) \mu c_{v}
$$

hence

$$
\frac{\lambda}{\rho_{0 m^{u} m^{r} m^{c} p l}}=\frac{(9 \gamma-5) \mu}{4 \gamma \rho_{0 m}{ }^{u} m_{m}^{r} m}
$$

This quantity is of the order of $10^{-4}$ for the systems of interest, while other quantities in the equation are of order unity, hence conduction is negligible. Omitting the conduction (third) term, and using Eq (10), we get,

$$
\frac{d}{d r^{*}}\left[\frac{T^{*}}{1+w}\right]=(\gamma-1) \frac{M_{t m}^{2}}{r^{* 3}}-g_{m} r^{*} n_{2}^{*}+\left(1-\frac{c_{p 2}}{c p l}\right) \frac{\rho_{2 m^{u}}{ }^{u}}{\rho_{0 m^{u}} u_{m}} \frac{d}{d r^{*}}\left(r^{*} q^{*} p_{2}^{*} u_{2}^{*}\right)
$$

This is the final form of the energy equation as it applies to the present problem. It may be put in a more convenient form by eliminating the derivative of $w$ from the left side and collecting the derivatives of T* from left and right sides. Thus, the density ratio, $w$, may be written,

$$
w=\frac{m_{2} n_{2}}{m_{1} n_{1}}=w_{m} \frac{n_{2}^{*} n^{*}}{p_{0}^{*}}
$$

whence

$$
\begin{array}{r}
\frac{d}{d r^{*}}(1+w)=w_{m} \frac{n_{2}^{*} T^{*}}{p_{0}^{*}}\left[\frac{1}{n_{2}^{*}} \frac{d n_{2}^{*}}{d r^{*}}+\frac{1}{T^{*}} \frac{d \mathrm{~T}^{*}}{d r^{*}}-\frac{1}{p_{0}^{*}} \frac{d p_{0}^{*}}{d r^{*}}\right], \\
200000
\end{array}
$$


and using Eq (10) to eliminate the $\mathrm{p}_{0}^{*}$ term,

$$
r^{*} \frac{d}{d r^{*}}(1+w)=w_{m} \frac{n_{2}^{* T^{*}}}{\dot{p}_{0}^{*}}\left[\frac{r^{*}}{n_{2}^{*}} \frac{d n^{*}}{d r^{*}}+\frac{r^{*}}{T^{*}} \frac{d T^{*}}{d r^{*}}-\gamma M_{t m}^{2} \frac{1+w}{r^{* 2} T^{*}}\right]:
$$

The left side of $\mathrm{Eq}$ (12) may now be expanded as follows,

$$
\begin{aligned}
\frac{d}{d r^{*}}\left[\frac{T^{*}}{1+w}\right]= & \frac{T^{*}}{(1+w)^{2}} \frac{1}{T^{*}} \frac{d T^{*}}{d r^{*}}-\frac{w}{(1+w)^{2}} T^{*} \frac{1}{n_{2}^{*}} \frac{d n_{2}^{*}}{d r^{*}}+ \\
& +\frac{\gamma M_{t m}^{2}}{r^{* 3}} \frac{w_{m}}{1+w} \frac{n_{2}^{*} T^{*}}{p_{\delta}^{*}}
\end{aligned}
$$

The derivative of $T^{*}$ may be separated from the right hand side of $\mathrm{Eq}(12)$, as follows:

$$
\frac{d}{d r^{*}}\left(r^{*} T^{*} n_{2}^{*} u_{2}^{*}\right)=r^{*} n_{2}^{*} u_{2}^{*} \frac{d T^{*}}{d r^{*}}+T^{*} \frac{d}{d r^{*}}\left(r^{*} n_{2}^{*} u_{2}^{*}\right)
$$


Substituting Eqs (14) and (15) in Eq (12) and collecting coefficients of the derivatives we get,

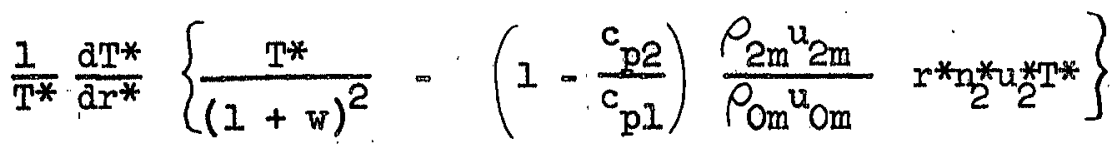

$$
\begin{aligned}
& -\frac{1}{n_{2}^{*}} \frac{d n_{2}^{*}}{d r^{*}}\left\{\frac{w}{(1+w)^{2}} T^{*}\right\}=\left(1-\frac{c_{p 2}}{c_{p I}}\right) \frac{\rho_{2 m^{u m}}}{\rho_{0 m^{u}} u^{*}} \frac{d}{d r^{*}}\left(r^{*} n_{2}^{*} u_{2}^{*}\right)= \\
& =\left\{\frac{\gamma-1}{\gamma}-\frac{w_{m}}{1+w} \frac{n_{2}^{*} T^{*}}{p_{0}^{*}}\right\} \frac{\gamma M_{t m}^{2}}{r^{* 3}}-g_{m} r^{*} n_{2}^{*}
\end{aligned}
$$

Finally, it is desirable to eliminate $u_{2 m} / u_{0 m}$ in favor of quantities which have more readily understandable physical significance. Let $M_{1}$ and $m_{2}$ be the mass flows of light gas and heavy gas per foot of tube length. Then

$$
\begin{aligned}
& m_{I}=-2 \pi r_{m} P_{I m}\left(u_{O m}+u_{I m}\right) \\
& m_{2}=-2 \pi r_{m} P_{2 m}\left(u_{O m}+u_{2 m}\right),
\end{aligned}
$$

and

$$
\rho_{I m} u_{I m}=-\rho_{2 m} u_{2 m}, \quad \text { or } \quad \frac{u_{I m}}{u_{2 m}}=-w_{m}
$$

hence

$$
\frac{m_{2}}{m_{I}}=w_{m} \frac{\frac{u_{O m}}{u_{2 m}}+I}{\frac{u_{O m}}{u_{2 m}}-w_{m}}
$$


Thus,

$$
\frac{u_{0 m}}{u_{2 m}}=\frac{w_{m}\left(1+\frac{m_{2}}{m_{1}}\right)}{\frac{m_{2}}{m_{1}}-w_{m}}
$$

Now by assumption $1, m_{2} / m_{1}=w_{c}$, where $w_{c}$ is the density ratio at the "exit" radius, $r_{c}$. Thus "

$$
\frac{u_{O m}}{u_{2 m}}=-\frac{1+w_{c}}{1-w_{c} / w_{m}}
$$

Using this relation, and noting that $\rho_{2 m} / \rho_{O m}=w_{m} /\left(1+w_{m}\right) \mathrm{Eq}(16)$ becomes,

$$
\begin{aligned}
& \frac{I}{T^{*}} \frac{d q^{*}}{d r^{*}}\left\{\frac{T^{*}}{(I+w)^{2}}+\left(1-\frac{c_{p}}{c_{p I}}\right)\left(\frac{w_{m}}{I+w_{m}}\right)\left(\frac{1-w_{c} / w_{m}}{I+w_{c}}\right) r^{*} n_{2}^{*} u_{2}^{*} T^{*}\right\} \\
& -\frac{1}{n_{2}^{*}} \frac{d n_{2}^{*}}{d r^{*}}\left\{\frac{w}{\left(1+w_{1}^{2}\right.} T^{*}\right\}+\left(1-\frac{c_{p 2}}{c_{p l}}\right)\left(\frac{w_{m}}{1+w_{m}}\right)\left(\frac{1-w_{c} / w_{m}}{1+w_{c}}\right), T^{*} \frac{d}{d r^{*}}\left(r^{*} n_{2}^{*} u_{2}^{*}\right)= \\
& =\left\{\frac{\gamma-1}{\gamma}-\frac{w_{m}}{1+w} \frac{n_{2}^{*} T^{*}}{p_{0}^{*}}\right\} \frac{\gamma M_{t m}^{2}}{r^{* 3}}-g_{m}{ }^{* *} n_{2}^{*}
\end{aligned}
$$

One expression relating the derivatives of $T^{*}, n_{2}^{*}$, and $r^{*} n_{2}^{*} u_{2}^{*}$ has thus been obtained from the energy equation." Two additional expressions relating these derivatives will be obtained from the diffusion equation and the conservation equation for the heavy gas. 
Conservation equation for the heavy gas:

From Eq (1), for $1=2$,

$$
\frac{d}{d r}\left(r n_{2} u_{2}\right)=-r u_{0} \frac{d n_{2}}{d r}-n_{2} \frac{d\left(u_{0} r\right)}{d r}
$$

From Eq (8), since $\rho_{0}=m_{1} n_{1}+m_{2} n_{2}$,

$$
\begin{aligned}
\frac{1}{u_{0} r} \frac{d\left(u_{0} r\right)}{d r} & =-\frac{1}{\rho_{0}} \frac{d \rho_{0}}{d r}=-\frac{1}{m_{1} n_{1}+m_{2} n_{2}}\left(m_{1} \frac{d n_{1}}{d r}+m_{2} \frac{d n_{2}}{d r}\right)= \\
& =-\frac{1}{1+w}\left(\frac{1}{n_{1}} \frac{d n_{1}}{d r}+w \frac{1}{n_{2}} \frac{d n_{2}}{d r}\right),
\end{aligned}
$$

and substitution into Eq (19) yields

$$
\frac{u_{2}}{u_{0}} \frac{1}{r n_{2} u_{2}} \frac{d}{d r}\left(r n_{2} u_{2}\right)=-\frac{1}{1+w} \cdot \frac{1}{n_{2}} \frac{d n_{2}}{d r}+\frac{1}{1+w} \frac{1}{n_{1}} \frac{d n_{1}}{d r}
$$


Referring all quantities to $r_{m}$ and using the equation of state to eliminate $n_{1}$ in terms of temperature and pressure

$$
\frac{u_{2 m}}{u_{0 m}} \frac{u_{2}^{*}}{u_{0}^{*}} \frac{1}{n_{2}^{*} u_{2}^{*}} \frac{d\left(r^{*} n_{2}^{*} u_{2}^{*}\right)}{d r^{*}}=-\frac{1}{1+w} \frac{r^{*}}{n_{2}^{*}} \frac{d n_{2}^{*}}{d r^{*}}+\frac{1}{1+w}\left[\frac{r^{*}}{p_{0}^{*}} \frac{d r_{0}^{*}}{d r^{*}}-\frac{r^{*}}{r^{*}} \frac{d r^{*}}{d r^{*}}\right] .
$$

Finally, using Eqs $(10)$ and (17) to eliminate $d p_{0}^{*} / \mathrm{dr}^{*}$ and $\mathrm{u}_{2 \mathrm{~m}} / \mathrm{u}_{\mathrm{Om}}$,

$$
\frac{d\left(r^{*} n_{2}^{*} u_{2}^{*}\right)}{d r^{*}}=-\frac{1+w_{c}}{1-w_{c} / w_{m}} u_{0}^{*} n_{2}^{*}\left\{\frac{\gamma M_{t m}^{2}}{r^{* 2} T^{*}}-\frac{1}{1+w}\left[\frac{r^{*}}{T^{*}} \frac{d r^{*}}{d r^{*}}+\frac{r^{*}}{n_{2}^{*}} \frac{d n^{*}}{d r^{*}}\right]\right\}
$$

This expression may be used to eliminate the derivative of $r^{*} n_{2}^{*} u_{2}^{*}$ from Eq (18).

A simple relation exists between $u_{2}^{*}, u_{0}^{*}$, and $w$, since Eq (19) may be written as

$$
\frac{d}{d r}\left(r n_{2} u_{2}\right)+\frac{d}{d r}\left(r n_{2} u_{0}\right)=0
$$

or

$$
r n_{2}\left(u_{0}+u_{2}\right)=\text { const. }
$$


Referring all quantities to their values at $r_{m}$ and using Eq (17), we get

$$
u_{2}^{*}=\frac{1+w_{c}}{1-w_{c} / w_{m}} u_{0}^{*}-\frac{w_{c}}{w_{m}} \frac{1+w_{m}}{I-w_{c} / w_{m}} \frac{1}{r^{*} n_{2}^{*}}
$$

Using the expressions for $w$ and $\rho_{0}$, this then becomes

$$
\frac{u_{2}^{*}}{u_{0}^{*}}=\frac{1-w_{c} / w}{1-w_{c} / w_{m}}
$$

Diffusion equation:

Dropping the term involving $K_{T}$, and using the fact that $n_{2} \ll n_{1}$, $\mathrm{Eq}$ (5) may be written,

$$
u_{1}-u_{2}=-D_{12}\left\{\frac{1}{n_{1}} \frac{d n_{1}}{d r}-\frac{1}{n_{2}} \frac{d n_{2}}{d r}+\left(\frac{m_{2}}{m_{1}}-1\right)\left(\frac{1}{1+w}\right) \frac{1}{p_{0}} \frac{d p_{0}}{d r}\right\} .
$$

Noting that $p_{0}=n_{1} k T$ and $u_{1}=-w u_{2}$, this becomes,

$$
u_{2}=\frac{D_{12}}{1+w} \cdot\left\{\left[\left(\frac{m_{2}}{m_{1}}-1\right)\left(\frac{1}{1+w}\right)+1\right] \frac{1}{p_{0}} \frac{d p_{0}}{d r}-\frac{1}{T} \frac{d T}{d r}-\frac{1}{n_{2}} \frac{d n_{2}}{d r}\right\}
$$


Referring all quantities to $r_{m}$, and using Eqs (10) and (17),

$$
\begin{aligned}
u_{2}^{*}= & -\frac{D_{12}}{r_{m^{u} O m}}\left(\frac{i+w_{c}}{1-w_{c} / w_{m}}\right)\left(\frac{1}{r^{*}}\right)\left\{\left(\frac{m_{2} / m_{1}-1}{-1+w}+1\right) \frac{M_{t m}^{2}}{r^{* 2} T^{*}}-\right. \\
& \left.-\frac{I}{1+w}\left[\frac{r^{*}}{T^{*}} \frac{d T^{*}}{d r^{*}}+\frac{r^{*}}{n_{2}^{*}} \frac{d n_{2}^{*}}{d r^{*}}\right]\right\}
\end{aligned}
$$

This is the third relation between the derivatives of $\mathrm{T}^{*}, \mathrm{n}_{2}^{*}$, and $\mathrm{r}^{*} \mathrm{n}_{2}^{*} \mathrm{u}_{2}$.

The diffusion coefficient:

The simple theoretical result for $D_{1}$, based on hard-sphere collisions, will be used, partly for the sake of simplicity and partly for lack of information about the ( 3 fteraction potentials for the molecules of interest. One such formula is,

$$
D_{12}=\frac{3}{8 n_{0} d_{12}^{2}} \cdot\left[\frac{k T\left(m_{1}+m_{2}\right)}{2 \pi m_{1} m_{2}}\right]^{\frac{1}{2}}
$$

where $d_{12}$ is the effective hard-sphere diameter for cqlision. Since $n_{0}=$ $\mathrm{p}_{0} / \mathrm{kT}$, lithis becomes,

$$
\mathrm{D}_{12}=\frac{3}{8} \frac{\mathrm{k}^{3 / 2}}{(2 \pi)^{1 / 2}}\left(\frac{1}{\mathrm{~m}_{1}^{1 / 2} \mathrm{~d}_{12}^{2}}\right)\left(1+\frac{\mathrm{m}_{1}}{\mathrm{~m}_{2}}\right)^{\frac{1}{2}} \frac{\mathrm{p}^{3 / 2}}{\mathrm{p}_{0}}
$$


Now the dimensionless group $D_{12} / r_{m} u_{0 m}$ becomes

$$
\frac{D_{12}}{r_{m}{ }^{u}}=\frac{3}{8} \frac{k^{3 / 2}}{(2 \pi)^{1 / 2}}\left(\frac{1}{m_{1}^{1 / 2} d_{12}^{2}}\right)\left(1+\frac{m_{1}}{m_{2}}\right)^{\frac{1}{2}} \frac{r_{m}^{3 / 2}}{r_{m}^{u_{0 m} p_{m}}} \frac{T^{* 3 / 2}}{p_{0}^{*}}
$$

We write this as,

$$
\frac{D_{12}}{r_{m} u_{m}}=-D_{m} \frac{T^{* 3 / 2}}{p_{0}^{*}}
$$

where

$$
\theta_{\mathrm{m}}-\frac{3}{8(2 \pi)^{1 / 2}}\left(\frac{\mathrm{k}^{3 / 2}}{\mathrm{~m}_{1}^{1 / 2} \mathrm{~d}_{12}^{2}}\right)\left(1+\frac{\mathrm{m}_{1}}{\mathrm{~m}_{2}}\right)^{\frac{1}{2}} \frac{\mathrm{T}_{\mathrm{m}}^{3 / 2}}{\mathrm{r}_{\mathrm{m}}^{\mathrm{u}} \mathrm{m}^{\mathrm{p}} \mathrm{Om}}
$$

Now

$$
r_{m} u_{O m} p_{O m} / T_{m}=r_{m} u_{m} P_{O m} \frac{I}{I+w_{m}} \frac{k}{m_{l}}
$$

and

$$
\begin{aligned}
m_{1}+m_{2} & =-2 \pi \rho_{O m} u_{m} r_{m}, \text { hence } \\
r_{m}{ }^{u} m^{p_{O m}} / T_{m} & =-\frac{m_{1}+m_{2}}{2 \pi} \frac{1}{1+w_{m}} \frac{k}{m_{1}}=-\frac{m_{1}}{2 \pi} \frac{1+w_{c}}{1+w_{m}} \frac{k}{m_{1}}
\end{aligned}
$$

Thus,

$$
D_{m}=\frac{3(2 \pi)^{\frac{1}{2}}}{8}\left(\frac{k^{1 / 2} m_{1}^{1 / 2}}{a_{12}^{2}}\right)\left(1+\frac{m_{1}}{m_{2}}\right)^{\frac{1}{2}}\left(\frac{1+w_{m}}{1+w_{c}}\right) \frac{T_{m}^{1 / 2}}{m_{1}}
$$


Solution for the derivatives of $\mathrm{n}_{2}^{*}$ and $\mathrm{T}^{*}$ :

Equations (18), (19), and (20) give three linear algebraic equations relating the derivatives of $T^{*}, n_{2}^{*}$, and $r^{*} n_{2}^{*} u_{2}^{*}$. These may be solved for the derivatives of $T^{*}$ and $n_{2}^{*}$, and the resulting two expressions, together with $\mathrm{Eq}(10)$, give three first-order non-linear differential equations, which may be integrated numerically. Thus, substituting $\mathrm{Eq}$ (20) in $\mathrm{Eq}$ (18), and collecting the coefficients of the derivatives,

$$
\begin{aligned}
& \frac{r^{*}}{T^{*}} \frac{d T^{*}}{d r^{*}}\left\{\frac{1}{(1+w)^{2}}+\left(1-\frac{c_{p 2}}{c_{p l}}\right)\left(\frac{w_{m}}{1+w_{m}}\right) r^{*} n_{2}^{*} u_{2}^{*}\left[\frac{1-w_{c} / w_{m}}{1+w_{c}}+\frac{u_{0}^{*}}{u_{2}^{*}} \frac{1}{1+w}\right]\right\}- \\
& -\frac{r^{*}}{n_{2}^{*}} \frac{d n_{2}^{*}}{d r^{*}}\left\{\frac{w}{(1+w)^{2}}-\left(1-\frac{c_{p 2}}{c_{p l}}\right) \frac{w_{m}}{1+w_{m}} \frac{r^{*} n_{2}^{*} u_{0}^{*}}{1+w^{\prime}}\right\}= \\
& =-g_{m} \frac{r^{* 2} n_{2}^{*}}{T^{*}}+\left\{\frac{\gamma-1}{\gamma}-\frac{w}{1+w}+\left(1-\frac{c_{p 2}}{c_{p l}}\right) \frac{w_{m}}{1+w_{m}} u_{0}^{*} n_{2}^{*} r^{*}\right\} \frac{\gamma M_{t m}^{2}}{r^{* 2} T^{*}}
\end{aligned}
$$

Equations (22) and (25) may now be written in the following form,

$$
\begin{aligned}
& a_{1} \frac{r^{*}}{T^{*}} \frac{d T^{*}}{d r^{*}}+b_{1} \frac{r^{*}}{n_{2}^{*}} \frac{d n_{2}^{*}}{d r^{*}}=c_{1} \\
& \frac{r^{*}}{T^{*}} \frac{d T^{*}}{d r^{*}}+\frac{r^{*}}{n_{2}^{*}} \frac{d n_{2}^{*}}{d r^{*}}=c_{2}
\end{aligned}
$$


where,

$$
\begin{aligned}
& a_{1}=\frac{1}{(1+w)^{2}}+\left(1-\frac{c_{p 2}}{c p 1}\right)\left(\frac{w_{m}}{1+w_{m}}\right) r^{*} n_{2}^{*} u_{2}^{*}\left[\frac{1-w_{c} / w_{m}}{1+w_{c}}+\frac{u_{0}^{*}}{u_{2}^{*}} \frac{1}{1+w}\right] \\
& b_{1}=\left(I-\frac{c_{p 2}}{c_{p 1}}\right) \frac{w_{m}}{I+w_{m}} \cdot \frac{r^{*} n_{2}^{*} u_{0}^{*}}{I+w}-\frac{w}{(1+w)^{2}} \\
& c_{1}=-g_{m} \frac{r^{* 2} n_{2}^{*}}{T^{*}}+\left\{\frac{\gamma-1}{\gamma}-\frac{w}{1+w}+\left(1-\frac{c_{p 2}}{c_{p l}}\right) \frac{w_{m}}{1+w_{m}} u_{0}^{*} n_{2}^{*} r^{*}\right\} \frac{\gamma M_{t m}^{2}}{r^{* 2} T^{*}} \\
& c_{2}=-\frac{1}{D_{m}} \frac{p_{0}^{*} T^{* 3 / 2}}{T^{* 3 / 2}} \frac{1-w_{c} / w_{m}}{I+w_{c}}(I+w)+\left(\frac{m_{2}}{m_{1}}+w\right)^{\frac{\gamma}{M_{t m}^{2}}} \frac{r^{* 2} T^{*}}{r^{2}}
\end{aligned}
$$

The derivatives may then be written,

$$
\begin{aligned}
& \frac{r^{*}}{T^{*}} \frac{d T^{*}}{d r^{*}}=\frac{c_{1}-c_{2} b_{1}}{a_{1}-b_{1}} \\
& \frac{r^{*}}{n_{2}^{*}} \frac{d n_{2}^{*}}{d r^{*}}=\frac{c_{2} a_{1}-c_{1}}{a_{1}-b_{1}}
\end{aligned}
$$

Equations (10), (27) and (28) may be integrated numerically. For convenience, Eq (10) will be repeated here.

$$
\frac{r^{*}}{p_{0}^{*}} \frac{d p_{0}^{*}}{d r^{*}}=\gamma M_{t m}^{2} \frac{1+w}{r^{* 2} T^{*}}
$$


The relations needed to complete the above set of equations are,

$$
\begin{aligned}
\rho_{0}^{*} & =\frac{1+w}{1+w_{m}} \frac{p_{0}^{*}}{T^{*}} \\
u_{0}^{*} & =1 / \rho_{0}^{*} r^{*} \\
\frac{u_{2}^{*}}{u_{0}^{*}} & =\frac{1-w_{c} / w^{\prime}}{1-w_{c} / w_{m}} \\
w & =w_{m} \frac{n_{2}^{*} T^{*}}{p_{0}^{*}}
\end{aligned}
$$

The parameters which must be specified, and which govern the process, are $\mathrm{g}_{\mathrm{m}}, \theta_{\mathrm{m}}, \mathrm{M}_{\mathrm{tm}}, \mathrm{w}_{\mathrm{m}}$, and $\mathrm{w}_{\mathrm{f}}$. All of the quantities with asterisks are by definition equal to unity at the radius $r=r\left(\right.$ or $r^{*}=1$ ) where $w$ is a maximum. The initial conditions for Eqs (10), ( 27 ) and (28) are therefore specified, and the integration can proceed numerically from $r^{*}=1$; however, the parameters $g_{m}, \theta_{m}, M_{t m}$, and $w_{m}$ are not all independent, if $w$ is to have a maximum at $r^{*}=1$. This wify be shown in the next section.

Maxima and Minima of w:

Because of the fact that the absolute value of $w$ enters $\mathrm{Eq}(26)$, rather than just the ratio of $\mathrm{w}$ to its value at some point in the tube; it is neces sary to specify the magnitude of $w$ at some value of $r^{*}$. This specified value of $\mathrm{W}$ Is an important parameter, since it effectively determines the fissionable material concentration in the tube. In order that the specified value of $w$ should be as accurate a measure of the average magnitude of $w$ as possible, it has been defined to be the maximum value of $w$ which occurs in the tube. However, 
"since the variation of $w$ with $r^{*}$ can be found only by numerical integration - of Eqs (10), (27) and (28), it is ... possible to specify the maximum of w only if it occurs at $r^{*}=1$, the initial point. In this section, conditions will be obtained which are necessary and sufficient for the occurrence of a maximum of $\mathrm{w}$ at $r^{*}=1$.

The condition that $d w / d r=0$ is, from Eq (13),

$$
\frac{r^{*}}{n_{2}^{*}} \frac{d n_{2}^{*}}{d r^{*}}+\frac{r^{*}}{T^{*}} \frac{d T^{*}}{d r^{*}}=\gamma M_{t m}^{2} \frac{1+w}{r^{*} T^{*}}
$$

Using Eqs (27) and (28) to eliminate the derivatives, this becomes,

$$
c_{2}=-\frac{1}{\mathcal{D}_{\mathrm{m}}} \frac{\mathrm{p}_{0}^{*} \mathrm{r}_{2}^{*}}{\mathrm{~T} * 3 / 2} \frac{1-\mathrm{w}_{\mathrm{c}} / \mathrm{w}_{\mathrm{m}}}{1+\mathrm{w}_{\mathrm{c}}}(1+w)+\left(\frac{\mathrm{m}_{2}}{\mathrm{~m}_{1}}+w\right)_{\mathrm{r}^{* \mathrm{~T}_{\mathrm{T}}^{*}}}^{\gamma \mathrm{M}_{\mathrm{tm}}^{2}}=\gamma \mathrm{M}_{\mathrm{tm}}^{2} \frac{1+\mathrm{w}}{\mathrm{r}^{* 2} \mathrm{~T}^{*}}
$$

Thus, the condition for zero slope of $\mathrm{w}$ at $\mathrm{r}^{*}=1$ is,

$$
D_{m}=\frac{\left(1-w_{c} / w_{m}\right)\left(1+w_{m}\right)}{\gamma M_{t m}^{2}\left(\frac{m_{2}}{m_{1}}-1\right)\left(1+w_{c}\right)}
$$

Equation (34) gives the value of $\theta_{m}$ such that $w$ shall have zero slope at $r^{*}=1$. With this value, $c_{2}$ becomes,

$$
c_{2}=\gamma M_{t m}^{2}\left[-\frac{p_{0}^{* r^{*} u_{2}^{*}}}{T^{* 3 / 2}}(1+w) \frac{\left(m_{2} / m_{1}-1\right)}{1+w_{m}}+\left(\frac{m_{2}}{m_{1}}+w\right) \frac{1}{r^{* 2} T^{*}}\right],
$$

and this expression replaces the corresponding one of Eqs (26). 
It is interesting to inquire whether there are points other than $r^{*}=1$ at which $\mathrm{Eq}$ (33) is satisfied. Substituting $\mathrm{Eq}(34)$ in $\mathrm{Eq}$ (33), we get,

$$
\frac{1+w}{1+w_{m}} \frac{p_{0}^{*} u_{2}^{*} r^{*}}{T^{* 1 / 2}}-\frac{1}{r^{* 2}}=0
$$

Now from $E q(31)$,

$$
\frac{u_{2}^{*}}{u_{0}^{*}}=\frac{1-w_{d} / w}{1-w_{0} / w_{m}} .
$$

$$
\text { Also, } p_{0}^{*}=\rho_{I^{*}}^{* T^{*}}=\frac{1+w_{m}}{I+w} \rho O^{* T^{*}} \text {, and } \rho_{0}^{*} u_{0}^{*} r^{*}=1 \text {, }
$$

hence, $\mathrm{Eq}(33 \mathrm{a})$ reduces to

$$
\frac{1-w_{c} / w_{1}}{I-w_{c} / w_{m}} T^{I / 2}=\frac{I}{r^{* 2}}
$$

which is the condition to be satisfied if there is to be a point of zero siope of $w$ other than at $r^{*}=1$. Since $T^{*}$ and $w$ are not known as functions of $r^{*}$ before the equation are integrated, it is not possible to give the points of zero slope of $w$ exactly: however, it is clear that if $\mathrm{Eq}$ (36) is to have a root for $r^{*}<1$, then the derivative of the left side with respect to $r^{*}$, evaluated at $r^{*}=1$, must be less than that of the right side, while the reverse is true if there is to be a root for $r * i$. 
It was found by integrating the equations that if there is a root for $r^{*}>1$, then $w$ is a maximum at $r^{*}=1$, while if there is a root for $r^{*}<1$, $w^{*}$ is a minimum at $r^{*}=1$. The behavior of $w$ is shown qualitatively for these two cases in Fig. 2. Clearly, the minimum slope of $T^{*}$ versus $r^{*}$ which is permissible if $\mathrm{w}$ is to have a maximum at $\mathrm{r}^{*}=1$ is such that the curve of the left side of $\mathrm{Eq}(36)$ versus $r^{*}$ is tangent to that of the right side at $r^{*}=1$. From Eq (36), this condition is

$$
\left.\frac{d r^{*}}{d r^{*}}\right|_{r^{*}=1}=-4
$$

From Eq (27),

$$
\left.\frac{d T^{*}}{d r^{*}}\right|_{r^{*}=1}=\frac{-g_{m}\left(1+w_{m}\right)+(\gamma-1) M_{t m}^{2}\left(1+w_{m}\right)}{1+\left(1-\frac{e_{p 2}}{c}\right) w_{m} \cdot \frac{I-w_{c} / w_{m}}{I+w_{c}}}=-4
$$

Solving for $\mathrm{g}_{\mathrm{m}}$,

$$
g_{m}(\max )=4\left[1+\left(1-\frac{c_{p 2}}{c_{p l}}\right) w_{m} \frac{1-w_{c} / w_{m}}{1+w_{c}}\right] \frac{1}{1+w_{m}}+(\gamma-1) M_{t m}^{2}
$$

The value of $\mathrm{g}_{\mathrm{m}}$ given by $\mathrm{Eq}(37)$ is to be interpreted as the maximum value, for given $w_{m}$ and $M_{t m}$, which allows $w$ to have a maximum at $r^{*}=1$. If $g_{m}$ is less than this value, $w / w_{m}$ will behave as in Fig. $2 b$, if Eq (36) has a root for $r^{*}>1$. However, if $\mathrm{Eq}^{\mathrm{m}}(36)$ does not have a root for $\mathrm{r}^{*}>\mathrm{I}, \mathrm{w} / \mathrm{w}_{\mathrm{m}}$ ill decrease continuously for $\mathrm{r}^{*}>1$.

This rather complicated behavior of the density ratio can perhaps be better understood by re-writing the diffusion equation in terms of $\mathrm{w}_{\circ}$ since $\mathrm{w} / \mathrm{w}_{\mathrm{m}}=$ $\mathrm{n}_{2}^{*} \mathrm{~T}^{*} / \mathrm{p}_{\mathrm{O}}^{*}$, we have

$$
\frac{r^{*}}{w} \frac{d w}{d r^{*}}=\frac{r^{*}}{n_{2}^{*}} \frac{d n_{2}^{*}}{d r^{*}}+\frac{r^{*}}{T^{*}} \frac{d T^{*}}{d r^{*}}-\frac{r^{*}}{p_{0}^{*}} \frac{d p_{0}^{*}}{d r^{*}}
$$


Now using Eqs (21) and (23), the diffusion equation [Eq (22)] may be written:

$$
I-\frac{w_{c}}{w}=\mathscr{D}_{m} \frac{1+w_{c}}{1+w_{m}} T^{* 1 / 2}\left\{\left(\frac{m_{2}}{m_{1}}-1\right) \frac{\gamma M_{t m}^{2}}{r^{* 2} T^{*}}-\frac{r^{*}}{w} \frac{d w}{d r^{*}}\right\}
$$

The first term in brackets on the right represents the vortex field; or rather its effect on the diffusion process, while the second represents the combined effects of the heavy and light gas concentrations. The influence of the radial. mass flow is contained in $\theta$, which is inversely proportional to the mass flow rate per unit of tube length, $M_{1}[$ see $(\mathrm{Bq}(24)]$

The physical significance of $\mathrm{Eq}(22 \mathrm{a}$ ) may be most easily seen by studying its form for three physical situations: the first with constant T* and no radial mass flow, the second with constant $T^{*}$ and radial mass flow, and the third with both strong temperature variation and radial mass flow.

If the mass flow rate is zero, $\mathcal{O}_{\mathrm{m}}$ is infinite [see Eq (24)], and Eq (22a) reduces to

$$
\frac{r^{*}}{w} \frac{d w}{d r^{*}}=\left(\frac{m_{2}}{m_{1}}-1\right) \frac{\gamma M_{t m}^{2}}{r^{* 2} r^{*}}
$$

Now if $T^{*}$ is constant; this immediately integrates to:

$$
\mathrm{w} / \mathrm{w}_{\mathrm{m}}=\exp \left[\left(\frac{\mathrm{m}_{2}}{\mathrm{~m}_{1}}-1\right) \frac{\gamma \mathrm{M}_{\mathrm{tm}}^{2}}{2 \mathrm{~T}^{*}}\left(1-\frac{1 \mathrm{c}}{\mathrm{r}^{* 2}}\right)\right] \text {. }
$$

Thus, for this case $w$ decreases very rapidly, and monotonically as $\mathrm{r}^{*}$ decreases from $\mathrm{r}^{*}=1$, as shown in Fig. 3 , case 1 . 
Next, suppose $T^{*}$ is still constant, but $M_{1}$ is not zero. Then unless $w$ is very small, $i_{0} e$. about equal to $w_{C}$, the quantity in braces in Eq (22a) must be equal to a positive constant, say $c_{9}$, which increases as $M_{I}$ increases. Now at $r *=1, d w / d r^{*}$ has been set equal to zero, hence the first term is just equal to $C$ for $r^{*}=1$. As $r^{*}$ decreases from unity, the first term increases, hence $d w / d r^{*}$ must become positive, while as $r^{*}$ increases, the first term decreases, and $\mathrm{dw}_{\mathrm{w}} / \mathrm{dr}^{*}$ must become negative in order that the sum of the two terms may remain equal to $C$. Thus, the radial mass flow produces a simple maximum in $\mathrm{w}$ as shown in Fig。 3 , case 2 .

Finally, consider the case in which there is both radial mass flow and sub stantial heat addition, which results in a decrease of $T^{*}$ as $r^{*}$ increases. The sum of the two terms in braces in $\mathrm{Eq}$ (22a) may still be considered roughly a constant, since $T^{*} 1 / 2$ varies rather slowly compared to the first term in the braces. This first term depends on both $\mathrm{r}^{*}$ and $\mathrm{T}^{*}$. If $\mathrm{T}^{*}$ varies slowly enough so that the product $r^{*} 2 T^{*}$ increases monotonically as $r *$ increases, the behavior of $\mathrm{w}$ is substantially the same as that of case 2. However, if W* $^{*}$ decreases more rapidly than $r^{* 2}$ increases, the first term increases with $r^{*}$ instead of decreasing, and $\mathrm{dw} / \mathrm{dr}$ must increase as $\mathrm{r}^{*}$ increases. If $\mathrm{r}^{*}$ is increased enough, $d w / d r$ becomes positive, and very large, because as $T^{*}$ decreases, the propellant density, and therefore the fissionable material density ( since $w \approx 1$ ) increase. The latter increase leads to a high rate of heat addition, which accelerates the decrease of $T^{*}$ with $r^{*}$, and the effect multiplies. Thus wirst decreases, then increases very rapidiy as shown by Fig 3 , case 3.

Whether the system behaves as in case 2 or as in case 3 depends on whether $T^{*}$ varies more rapidiy than $r^{*} 2$ for some $r^{*}$ greater than unity。 Now the variation of $\mathrm{T}^{*}$ with $\mathrm{r}^{*}$ is proportional to $\mathrm{g}_{\mathrm{m}}$, at least for $\mathrm{r}^{*}$ near unity, thus as $g_{m}$ is increased from zero, the behavior of $w$ will change from that of the second case to that of the third. The value of $g_{m}$ at which this change in. behavior takes place will be denoted $g_{m}$ (crit).

In summary, the conditions which must be satisfied in order that $w$ have a maximum point at $r^{*}=1$ are that $c_{2}$ be given by $\mathrm{Eq}$ (35) and that $\mathrm{g}_{\mathrm{m}}$ be less than the value $g_{m}(\max )$ given by $E q(37)$. If $g_{m}$ is between $g_{m}(\max )$ and $g_{m}$ (crit) w will behave as shown in Fig 3 for case 3 . If $g_{m}$ is less than $g_{m}$ (crit), w will behave as shown for case 2 . 
Method of Integration:

Equations (10), (27), (28), (29), (30), (31), and (32), with Eq (35) for $c_{2}$, are in such a form that they may be integrated quite readily by a highspeed digital computor. Since only the first derivatives of T* and are easily available, a simple forward-difference scheme was used, and the desired precision obtained by means of a small interval.

Beginning at the initial point, $r^{*}=1$ where $n_{2}^{*}=T^{*}=p^{*}=\rho_{0}^{*}=u_{0}^{*}=u_{2}^{*}=1$, and $\mathrm{w}=\mathrm{w}_{\mathrm{m}}$, the quantities

$$
\left(r^{*} / \mathrm{n}_{2}^{*}\right) \mathrm{d} \mathrm{n}_{2}^{*} / \mathrm{d} \mathrm{r}^{*}, \quad\left(\mathrm{r}^{*} / \mathrm{T}^{*}\right) \mathrm{d} \mathrm{T}^{*} / \mathrm{d} \mathrm{r}^{*}, \quad\left(\mathrm{r}^{*} / \mathrm{p}^{*}\right) \mathrm{d} \mathrm{p} / \mathrm{d} \mathrm{r}^{*}
$$

were computed. Values of $n^{*}, T^{*}$ and $p_{0}^{*}$ were then estimated at the next value of $\mathrm{r}^{*}$ by the relation,

$$
y_{i+1}=y_{i} \exp \left[\left.\frac{r_{i}^{*}}{y} \frac{d y}{d r^{*}}\right|_{r_{i}^{*}} \frac{r_{i+1}^{*} r_{i}^{*}}{r_{i}^{*}}\right]
$$

where $\mathrm{y}=\mathrm{n}^{*}, \mathrm{~T}^{*}, \mathrm{p}_{0}^{*}$. With these values, $\mathrm{P}_{0}^{*}, \mathrm{u}_{0}^{*}, \mathrm{u}_{0}^{*}$, and $\mathrm{w}$ were then estimated。 The derivatives at the end of the interval were then computed from these values, and final values for each of the above derivatives were obtained by averaging the estimated values with the corresponding values at the beginning of the interval. The final values of $n_{2}^{*}, T^{*}$ and $p^{*}$ were then computed by $\mathrm{Eq}(38)$, using these average derivatives. This procedure was repeated for each increment in $r *$

An interval of $\Delta r^{*}=0.005$ was used for all the calculations. In order to estimate the errors, one case was run.with an interval of 0.01 . Since the results for the quantities of interest agreed to within one percent, it was concluded that the accuracy was sufficient for engineering purposes.

Parameters of the heating-separation process:

For each integration of the differential equations, $M_{t m}, w_{m}, g_{m}$, and $w_{f}$ were specified. The integration then proceeded from $r^{*}=1$ toward small $r^{*}$. The integration was stopped when $w$ equalled $w_{\text {, }}$ and the value of $r^{*}$ at which this occurred was called r. For all of the calculations $\mathrm{w}_{\mathrm{c}}=0.0001$ was selected. This figure implies a loss of 0.0001 pounds of fissionable material for each pound of propellant expended. It could be reduced to 0.00001 without qualitatively altering the results. 
The parameters which remain are then $M_{t m}, w^{\prime}$ and $g_{m}$. For any particular set of these, the integration gave the variation of $n_{2}^{*}, T^{*}, \rho_{0}^{*}$, and $w$ with $r^{*}$, for $r^{*}$ in the range where $w$ was less than or equal to $w_{m^{\circ}}$

In order to interpret the results in terms of real systems it is necessary first to select the value of $\mathrm{r}^{*}$ which is to represent the periphery of the tube, i.e. r. Each solution for a given set of $M_{t m}, w_{m}$ and $g_{m}$ allows a range of values of $r_{p}^{*}$, and the overall characteristics of the system depend strongly on $r$. The values of $r *$, have been chosen for the cases where $g_{m}>g_{m}$ (crit) so as to include the entire range of $r^{*}$ for which $w \leq w_{m^{*}}$ By reference to Fig. 3 it may be seen that $r_{p}^{*}$ is the value of $r^{*}$ at which $\frac{\mathrm{m}}{\mathrm{w}} / \mathrm{w}_{\mathrm{m}}$ becomes unity again as $r^{*}$ is increased from unity. For the cases where $g_{m_{1}} g_{m}$ (crit), $r_{p}^{*}$ has been selected as the value of $\mathrm{r}^{*}$ at which $\mathrm{w}=0.1 \mathrm{w}_{\mathrm{m}^{\circ}} \mathrm{m}_{\mathrm{It}} \mathrm{w}_{\mathrm{ill}}$ be seen later that this choice gives approximately the maximum overall temperature ratio, from tube exit to periphery.

For a given value of $r_{p}^{*}$, the tangential Mach number, $M_{t p}$, and the heating parameter, $\delta_{p}$, both referfed to the fluid conditions at the tube periphery, may be computed. From the definition of the Mach number,

$$
M_{t p}=\frac{v_{0 p}}{\left(\gamma R p_{p}\right)^{1 / 2}}
$$

Using Eq (2), this becomes,

$$
M_{t p}=M_{t m} \frac{1}{r_{p}^{*} T_{p}^{*}}
$$


Similarly; from the definition of $\mathrm{g}$,

$$
g_{p}=-\frac{\sigma_{f} \phi Q n_{2 p} r_{p}}{c_{p I} \rho_{O p}{ }^{u} O p^{T}}=2 \pi \frac{n_{2 p} \sigma_{f} \phi Q r_{p}^{2}}{m_{1}\left(1+w_{c}\right) c_{p I}{ }^{T}},
$$

and

$$
g_{p}=g_{m} \frac{n_{2 p}^{*} r_{p}^{* 2}}{T_{p}^{*}}
$$

The physical significance of $M_{t p}$ is of course clear. It is simply the velocity of the entering fluid divided by its sonic velocity. Other things being equal, the strength of the vortex field increases as $\mathrm{M}_{\mathrm{tp}}$ increases.

From Eq. (40), it is clear that $g_{p}$ measures the ratio of the heat generation rate by fission per unit of tube length to the heat capacity. of the fluid flowing through the tube, per unit length and per unit time. It is therefore a direct measure of the temperature ratio across the tube, from periphery to core. In the process of integration, $\mathrm{g}_{\mathrm{m}}$ was fixed, and for each $r_{p}^{*}$ a value of $g_{p}$ was computed, from $E q .(41)$. This value of $g_{p}$ determines the relationship necessary between the parameters of $\mathrm{Eq}$. (40) in order that the heat generation rate be that which is implied by the resultant temperature ratio, $\mathrm{T}_{\mathrm{c}} / \mathrm{T}_{\mathrm{p}}$. 
A relationship between the mass flow rate per unit of tube length, $M_{I}$, and the other parameters is implied by Eqs (24) and (34). Equating these and solving for $M_{1}$, we get

$$
m_{1}=\frac{3(2 \pi)^{1 / 2}}{8} \frac{k^{1 / 2} m_{1}^{1 / 2}}{d_{12}^{2}}\left(1+\frac{m_{1}}{m_{2}}\right)^{\frac{1}{2}}\left(\frac{m_{2}}{m_{1}}-1\right) \frac{\gamma_{p}^{1 / 2}}{\left(1-w_{c} / w_{m}\right)} \Psi
$$

where

$$
\Psi=\frac{M_{t m}^{2}}{T_{p}^{* 1 / 2}}
$$

Since $T_{p}^{1 / 2} / T_{p}^{* 1 / 2}=T_{m}^{1 / 2}$, the mass flow rate depends on $T_{m}, M_{t m}$, and constants determined by the lightaheavy gas combination. It is proportional to $\mathrm{T}_{\mathrm{m}}^{1 / 2} \mathrm{M}_{\mathrm{tm}}^{2}$

It is important to note that $M$ is the mass flow capacity per unit of tube length, and that it is independent of the tube size. Thus, a large number of small diameter tubes filling a given volume have a much higher mass flow capacity than one large tube of the same volume. This is felt to be one of the most imm portant results of the analysis.

In order to estimate the critical size of a system composed of vortex tubes, it is necessary to know the average densities of the light and fissionable gases in the vortex tube. These may be obtained by averaging over the density distributions given by the integration. 
If $r_{c}^{*}$ is the exit dimensionless radius and $r_{*}^{*}$ is the peripheral dimensionless radius, and it is assumed $P_{1}$ is constant for $r * r^{*}$, and has the value $P_{1 c}$, then
the ratio of average light gas density to that at the periphery is;

$$
\frac{\bar{\rho}_{1}}{\rho_{1 p}}=\frac{\rho_{1 c^{*}}^{r_{c}^{*}}+2 \int_{r_{\varepsilon}^{*}}^{r_{p}^{*}} \rho_{1}^{*} r^{*} r^{*}}{\rho_{1 p_{p}^{*} r_{p}^{* 2}}}
$$

or

$$
\frac{\bar{\rho}_{I}}{\rho_{1 p}}=\frac{p_{0 c}^{*} T_{p}^{*}{ }_{p}^{* 2}}{p_{O p}^{*} T_{c}^{*}{ }_{p}^{* 2}}+2 \frac{T_{p}^{*}}{p_{O p}^{*} r_{p}^{* 2}} \int_{r_{c}^{*}}^{r_{p}^{*}} \frac{p_{0}^{*}}{T^{*}} r^{*} d r^{*}
$$

If it is assumed that $w$ is very small for $r^{*}<r_{c}^{*}$, the average density ratio $\bar{w}$, defined as $w \bar{\rho}_{2} / \bar{\rho}_{1}$, is given by,

$$
\bar{w}=\frac{2 w_{m}}{r_{p}^{* 2}} \frac{\rho_{l p}}{\rho_{I}}\left(\frac{T_{p}^{*}}{p_{0 p}^{*}}\right) \int_{r_{c}^{*}}^{r_{p}^{*}} n_{2}^{*}{ }^{* d r} d r^{*}
$$

The average density of the fissionable gas in the tube is then given by

$$
\overline{\rho_{2}}=\bar{w}\left(\frac{\bar{\rho}_{1}}{\rho_{1 p}}\right) \rho_{1 p} .
$$


In summary, the following parameters are significant in specifying the overall performance of the heating-separation process: the overall temperature ratio, $\mathrm{T}_{\mathrm{c}} / \mathrm{T}_{\mathrm{p}}$, the overall pressure ratio, $\mathrm{p}_{\mathrm{O}} / \mathrm{p}$ op, the peripheral tangential Mach number, $M_{t p}$, the outlet-to-peripheral radius ratio, $r_{c} / r_{p}$, and the relative mass flow capacity per unit of tube length, $\Psi$. The coupling between the fluid-mechanical and nuclear characteristics of the system is expressed by the average fissionable-species-to-propellant-density ratio, $\varpi$, the average-to-peripheral propellant density ratio, $\bar{P}_{1} / \rho_{1 p}$, and the heating parameter, $g_{p}$, which is essentially a statement on the required neutron track length.

Results:

The principal results of the analysis take the form of relationships between the overall-performance and nuclear-coupling parameters and the specified parameters, $\mathrm{w}_{\mathrm{m}}, \mathrm{M}_{\mathrm{tm}}$, and $\mathrm{g}_{\mathrm{m}}$; however, it is difficult to understand these relationships without a prior understanding of the physical aspects of the heating separation process. Accordingly, before these principle results are presented, the variation of the several physical parameters with $r^{*}$ will be presented for some typical values of the specified parameters. Because of the large number of possible combinations of the specified parameters, these results can be only exemplary.

The calculations have been done for a combination of hydrogen as the light gas and plutonium as the heavy gas. The quantities involved in the integration which depend on this choice, and the assigned values are: $\mathrm{m}_{2} / \mathrm{m}_{1}=119.5$, $c_{p 2} / c_{p 1} \approx 0.008, \gamma=1.31$. The value of $\gamma$ was selected as a reasonable mean for the temperature range of interest.

The density ratio, dimensionless temperature, and dimensionless fuel concentration:

The somewhat complicated behavior of $\mathrm{w}$, which has been discussed qualitatively at some length in the section "maxima and minima of $\mathrm{w}^{\prime \prime}$, is shown quantitatively in Figs $4 a$ and $4 b$, for typical values of $M_{t m}$ and $w_{m}$. For $g_{m}<g_{m}$ (crit), (Fig 4a), w has a simple maximum, and falls off quite rapidly for $r *$ both greater than and less than unity. This simple maximum results from the fact that for $g_{m}<g_{m}$ (crit), the vortex field strength decreases monotonically as $r$ increases. If, on the other hand, the rate of heat addition is large enough, i.e. $g_{m}>g_{m}$ (crit) the rapid increase in density as $r$ increases causes the field strength first to decrease, then increase again as $r$ increases. In these cases, $w$ increases again as the field strength increases, as shown in Fig 4b. 
The variation of $T^{*}$ is shown for the same values of $M_{t m}, w_{m}$, and $g_{m}$ in Figs. $5 a$ and $5 b$. For $g<g_{m}(c r i t), T^{*}$ varies rather slowly. Near $r^{*}=1$, where $\mathrm{w} / \mathrm{w}_{\mathrm{m}}$ is near unity, $\mathrm{T}^{\mathrm{m}}$ increases steadily; however, as $\mathrm{r}^{*}$ becomes small, and $\mathrm{w}$ becomes small, $\mathrm{T}^{*}$ decreases because the propellant expands toward the center of the tube. Similarly, if $r^{*}$ were carried to large enough values, $T^{*}$ would increase as $r^{*}$ increased for $r^{*}>1$.

For $g_{m}>g_{m}$ (crit), the variation of $T^{*}$ reflects the fact that $w / w_{m}$ is of order unity for all $r^{*}>1$. $T^{*}$ continues to decrease as $r^{*}$ increases, and in fact for $g_{m}$ near $g_{m}$ (crit), $T *$ decreases very rapidly as $w$ increases rapidly. As has been pointed out previously, these two effects reinforce each other, since as the temperature decreases, the derivative of $w$ in. creases, which gives a higher rate of heat addition and decreases T $^{*}$ further.

The variation of the dimensionless fissionable-species concentration with $r *$ is shown in Figs $6 a$ and $6 b$ for the cases discussed above. For $g_{m}<g_{m}$ (crit), $n$ is very similar to $w$ (compare Figs $6 a$ and $4 a$ ), while for $g_{m}>g_{m}(c r i t)$ the large variations in propellant density, which result from the large variations in $T^{*}$, cause a rather extreme variation of no near the periphery of the tube for values of $g_{m}$ near $g_{m}$ (crit).

The validity of the analysis for these extreme cases where $g_{m}$ is near $g_{m}($ crit) will be discussed in a later section.

All of the above results have been for $w_{m}=1.0$ and $M_{t m}=0.7$. The in fluence of $\mathrm{w}_{\mathrm{m}}$ on the distribution of $\mathrm{w}$ is shown in Fig 7. The effect is small, even when $w_{m}$ is changed by a factor of 20 .

On the other hand; Fig. 8 shows that $M_{t m}$ has a very strong influence on the distribution of fissionable material. As $\mathrm{M}_{t m}$ is increased, $w$ varies more rapidly about its fixed maximum, $w_{m}$. This is due to the fact that the vortex field strength increases with $\mathrm{M}_{\mathrm{tm}}$ 。

Dependence of the performance parameters on the choice of $r_{p}^{*}$ :

Because any value of $r^{*}$ may be chosen to represent the periphery of the tube for a given set of $\mathrm{M}_{t m}$, $\mathrm{w}_{\mathrm{m}}, \mathrm{g}_{\mathrm{m}}$, each choice for these parameters yields a very wide range of possible physical systems. In order to make the results as concrete as possible, $r$ has been chosen as follows. For $g_{m}<g_{m}$ (crit), $r *$ has been taken as that value of. $\mathrm{r}^{*}$ which gives the largest $\mathrm{T}_{c} / \mathrm{T}_{\mathrm{p}^{\circ}}$ The variation of $\mathrm{T}_{c} / \mathrm{T}_{\mathrm{p}}$ with the choice of $r_{p}^{*}$ is shown for these cases in Fig $9 a$, together with that of 
poc/POp. For $g_{m}>g_{m}$ (crit), $r_{p}^{*}$ has been selected to include the entire region of $r^{*}$ in which $\mathrm{w}<\mathrm{w}_{\mathrm{m}}$. This choice also yields the largest value of $\mathrm{T}_{c} / \mathrm{T}_{\mathrm{p}}$ consistent with the requirements that $w<w_{m}$ as shown in Fig $9 \mathrm{~b}$. It is true that if, for example, a temperature ratio, $\mathrm{T}_{\mathrm{p}} / \mathrm{T}_{c}=0.7$ is desired, it may be obtained for any of the values of $g_{m} / g_{m}(\max )$ shown in Fig $9 b$, by choosing $r$ * suitably; however, the presentation of the results would have become very cumbersome had a.II such possibilities been included, and it is felt that the above somewhat arbitrary choices for $r *$ show the general characteristics of the heating - separation process to best advantage.

The dependence of the peripheral Mach number, $M_{t p}$, on the choice of $r_{p}^{*}$ is shown in Fig 10. For $g_{m}<g_{m}$ (crit), $M_{t p}$ decreases as $r$ increases, reflecting the decrease in tangential velocity as $r$ increases. For $g_{m}>g_{m}$ (crit), $M_{t p}$ increases as $r$ increases. This reversal is due to the more rapid decrease in temperature, and hence, in the velocity of sound, for these cases, the velocity of sound actually decreasing faster than the tangential velocity.

Another performance parameter of considerable interest is $\Psi$, the relative mass flow capacity per unit of tube length. Figure 11 shows that it increases with $r_{p}^{*}$ for $g_{m}$ both greater than and less then $g_{m}$ (crit), though much more rapidif for the former. It should be noted that the actual mass flow per unit of tube length, $M_{1}$, is proportional to $\Psi$ times $\mathrm{T}_{\mathrm{p}} 1 / 2$. Therefore, the very rapid rise in $\Psi$ for $g_{m} / g_{m}(\max )=0.51$ implies a correspondingly rapid increase in $W_{1}$ only if $\mathrm{T}_{\mathrm{p}}$ is held constant. If $\mathrm{T}_{\mathrm{c}}$ is held constant, $\mathrm{T}_{\mathrm{p}}$ decreases as $\mathrm{T}_{\mathrm{p}} / \mathrm{T}_{\mathrm{c}}$ decreases, and $M_{1}$ actually decreases somewhat as $r_{p}^{*}$ increases.

Dependence of the nuclear coupling parameters on the choice of $\mathrm{r}_{\mathrm{p}}^{*}$ :

The quantities which are needed in computing the criticality of a system of vortex tubes are the average fissionable-species and propellant densities. The ratio of average-tomperipheral propellant densities is shown as a function of $r$ in Figs $12 \mathrm{a}$ and $12 \mathrm{~b}$, together with the ratio of average-to-maximum of the density ratio, wo Both of these parameters are rather insensitive to $\mathrm{r}_{\mathrm{p}}^{*}$ for $\mathrm{g}_{\mathrm{m}}<\mathrm{g}_{\mathrm{m}}$ (crit), with the maximum $\overline{\mathrm{w}} / \mathrm{w}_{\mathrm{m}}$ occurring for a somewhat smaller $\mathrm{r}_{\mathrm{p}}^{*}$ than that for maximum $T_{c} / T_{p}$. Thus, if the largest possible value of $\bar{w} / w_{m}$ were desired, rather than the largest $\mathrm{T}_{c} / \mathrm{T}_{\mathrm{p}}$, $\mathrm{r}_{\mathrm{p}}^{*}$ would be modified somewhat. For $\mathrm{g}_{\mathrm{m}}>\mathrm{g}_{\mathrm{m}}$ (crit), both $\bar{w} / \mathrm{w}_{\mathrm{m}}$ and $\bar{\rho}_{1} / \rho_{1 \mathrm{p}}$ are more sensitive to the choice of $\mathrm{r}_{\mathrm{p}}$

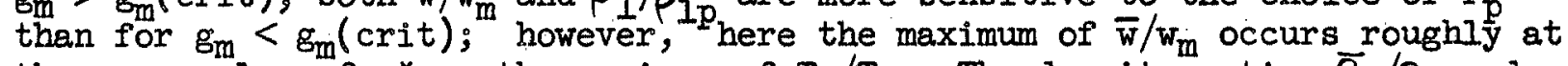
the same value of $r_{p}^{*}$ as the maximum of $T_{c} / T_{p}$. The density ratio, $\bar{P}_{1} / P_{1} p^{2}$ decreases steadily as $r_{p}^{*}$ increases. This is simply a reflection of the increasing ratio of average temperature to peripheral temperature. 
Finally, the parameter $g_{p}$, which essentially determines the required neutron track length, is shown in Figs 13a and 13b. It increases rapidiy with $r$ for $\mathrm{g}_{\mathrm{m}}>\mathrm{g}_{\mathrm{m}}$ (crit), reflecting the higher rate of heat release demanded by the higher temperature ratios. For $g_{m}<g_{m}$ (crit), $g_{p}$ decreases as $r_{p}^{*}$ increases, instead of increasing, even though the temperatore ratio increases with $r$. This is reasonable, since $g_{p}$ is proportional to nkp times $\phi$, and $n$, decreases as $r_{p}^{*}$ increases.

The overall temperature ratio:

Of the several performance parameters, the overall temperature ratio is perhaps the best measure of the performance of the system, at least for rocket applications. Therefore, it has been selected as a basic variable for presen: ting the results. Its dependence on the heating parameter will first be shown, then all other performance parameters will be referred to it.

A curve of $\mathrm{T}_{\mathrm{p}} / \mathrm{T}_{\mathrm{c}}$ versus $\mathrm{g}_{\mathrm{m}} / \mathrm{g}_{\mathrm{m}}(\max )$ for constant $\mathrm{M}_{\mathrm{tm}}$ and $\mathrm{w}_{\mathrm{m}}$ has two branches, corresponding to the two cases, $g_{m}<g_{m}(c r i t)$ and $g_{m}>g_{m}$ (crit), as shown in Fig 14. Modest temperature ratios can be obtained on either branch; however, in order to obtain values of $\mathrm{T}_{\mathrm{p}} / \mathrm{T}_{\mathrm{c}}$ less than about 0.3 it appears essential that $\mathrm{gm}$ be greater than $\mathrm{g}_{\mathrm{m}}$ (crit). There appears to be no limit to the temperam ture ratio obtainable by approaching $g_{m}$ (crit) from above. Although the endpoints of the curves for $g_{m}<g_{m}$ (crit) were not very well defined by the calculations, it is believed that the end points as shown are approximately. correct. That there is a minimum obtainable $T_{p} / T_{c}$ for $g_{m}<g_{m}(c r i t)$, and not for $g_{m}>$ $\mathrm{gm}$ (crit) is due to the sudden rise in w near the periphery in the latter case, and the lack of it in the former. This rise in wives a high fuel concentration near the periphery which leads to a very rapid temperature variation, and the small values of $\mathrm{T}_{\mathrm{p}} / \mathrm{T}_{c}$ shown for $\mathrm{gm}_{\mathrm{m}}>\mathrm{g}_{\mathrm{m}}$ (crit). For $\mathrm{g}_{\mathrm{m}}<\mathrm{g}_{\mathrm{m}}$ (crit) on the other hand, w decreases steadily to the periphery, giving a very gradual temperature variation.

From a comparison of Figs $14 \mathrm{a}, \mathrm{b}$ and $\mathrm{c}$, it can be seen that the variation of $\mathrm{T}_{\mathrm{p}} / \mathrm{T}_{\mathrm{c}}$ with $\mathrm{g}_{\mathrm{m}} / \mathrm{g}_{\mathrm{m}}(\max )$ is more rapid as $\mathrm{M}_{t m}$ increases. This is due to the more abrupt variation of the concentration profiles with increasing $\mathrm{M}_{\mathrm{tm}}$, which in turn is due to the higher vortex field strength. 
The overall pressure ratio:

As the overall temperature ratio is a measure of the performance of the vortex tube as a heating -separation device, the overall pressure ratio is a measure of the penalty paid for the separation. The pressure drop results, of course, from the resistance which must be overcome by the propellant in diffusing through the heavy gas; therefore, the more dense the heavy gas cloud is, the larger the pressure drop may be expected to be. That this is true may be seen from Figs $15 \mathrm{a} ; \mathrm{b}$ and $c$.

It may also be seen that the pressure ratio decreases as $T_{p} / T_{c}$ decreases. This is due to increased expansion of the gas toward the center of the tube as the temperature rise increases.

The pressure ratio also decreases as $\mathrm{M}_{\text {tm }}$ is increased, because of the increasing vortex field strength, whlch tends to concentrate the propellant near the periphery of the tube, as well as the heavy gas.

The peripheral Mach number:

Although $M_{t m}$ was specified in carrying out the calculations, the parameter which is of interest in an actual system is $M_{t p}$, the Mach number at the periphery, since it is the Mach number at which the propellant enters the vortex tube.

The variation of $\mathrm{M}_{t p}$ with $\mathrm{T}_{\mathrm{p}} / \mathrm{T}_{\mathrm{c}}$ is shown in Fig 16 for various values of $\mathrm{M}_{\mathrm{tm}}$. The dependence of this relationship on $\mathrm{w}_{\mathrm{m}}$ was found to be small, and so is not shown:

As $\mathrm{T}_{\mathrm{p}} / \mathrm{T}_{\mathrm{c}}$ is decreased, larger values of $\mathrm{M}_{\mathrm{tp}}$ are required to give a specified value of $M_{t m}$ since the velocity of sound at the periphery becomes smaller compared to that at $r_{m}$. Another interpretation of this same effect is that if $\mathrm{T}_{\mathrm{c}}$ is held constant, larger values of $\mathrm{M}_{t p}$ are required to generate a given vortex field strength (or given $\mathrm{M}_{\mathrm{tm}}$ ) as $\mathrm{T}_{\mathrm{p}}$ decreases. Thus, for a given vortex field strength, larger pressure drops must be maintained in the inlet nozzles as $\mathrm{T}_{\mathrm{p}} / \mathrm{T}_{\mathrm{c}}$ is decreased. These pressure ratios must be combined with those shown in Fig 15 to obtain the effective pressure ratio across the entire system. 
The relative mass flow capacity:

of equal importance to the temperature ratio is the mass-flow capacity of the vortex tube, which actually determines the total tube length required for a given total mass flow or thrust.

The vortex tube is rather unusual, in that the mass flow capacity per unit of tube length is independent of the tube diameter. This fact cannot be too strongly emphasized. It means that the mass flow capacity per unit of tube volume, which is a measure of the system size for a given mass flow, is inversely proportional to the tube diameter, within limits to be indicated in the next section.

From Fig 17, it may be seen that the relative mass flow capacity per unit of tube length increases as $\mathrm{M}_{t m}$ increases. It is in fact nearly proportional. to $\mathrm{MEm}_{\mathrm{m}}$. The dependence on temperature ratio is not so clear, since $\Psi$ must be multiplied by $\mathrm{T}_{\mathrm{p}} \mathrm{I} / 2$ to obtain the actual mass flow capacity per unit of tube length. If ' $T_{p}$ is held constant, and $T_{c}$ increased, the mass flow capacity increases; however, a simple computation with the aid of these curves will show that if $\mathrm{T}_{\mathrm{c}}$ is held constant, while $\mathrm{T}_{\mathrm{p}}$ is decreased, the mass flow capacity actually decreases slightly.

Numerical values of $M_{1}$, the actual mass flow capacity per unit tube length, will be given for some sample cases in a later section (see Eq 42).

The exit-to-peripheral radius ratio:

In all of the preceding results, it is implied that $w$ is reduced to $a$ small value, $w_{C}$, at some radius, $r_{c}$, within the tube, and that the propellant leaves the tube at this radius, with only a very small amount of heavy gas. As was mentioned previously, $w_{C}=0.0001$ was selected for all of the present calculations.

The resulting values of the exit-to-peripheral radius ratio are shown in Fig 18. As might be expected, this ratio increases as $\mathrm{M}_{\mathrm{tm}}$ increases, since the concentration profiles become more abrupt as $\mathrm{M}_{t m}$ increases. It also increases as $\mathrm{T}_{\mathrm{p}} / \mathrm{T}_{\mathrm{c}}$ increases, because heating of the propellant increases its radial velocity, which tends to sweep the heavy gas toward the center of the tube. 
From the standpoint of the overall performance of the vortex tube as a rocket propulsion device, the significance of $r_{c} / r_{p}$ is that it sets an upper limit on the ratio of exit. nozzle throat radius to tube peripheral radius. Referring to Fig 1 , it is clear that if the throat to tube radius ratio, say $r_{t} / r_{p}$, is greater than $r_{c} / r_{p}$, the cloud of fissionable material will be swept from the tube. If $r_{t} / r_{p}$ is less than $r_{c} / r_{p}$, the results of the calculations are in some sense conservative, since then; the actual value of $w$ in the exhaust nozzle may be somewhat less than $\mathrm{w}_{c}$. For a given set of separation parameters, the mass flow capacity of a vortex tube is simply $M_{1} I_{\text {, where }} L$ is the tube length, and the throat radius is determined by this mass flow. A lower limit on the tube radius is then set by the fact that $r_{c}$ must not be less than $r_{t}$. Numerical examples of this relationship will be given in a later section.

\section{Nuclear coupling parameters:}

In order to estimate the critical size of a system of vortex tubes it is necessary to know the average fissionable and light gas concentrations in the vortex tubes. These may be obtained from $\mathrm{w} / \mathrm{w}$, the ratio of average to maximum density ratios, and $P_{I} / \rho_{l p}$, the ratio of average to peripheral propellant densities.

The dependence of $\bar{w} / w_{m}$ on $T_{p} / T_{c}, W_{m}$ and $M_{t m}$ is shown in Fig 19. For given $w_{m}$ and $M_{t m}$, if $g_{m}>g_{m}$ (crit), there is a value of $w_{p} / T_{c}$ which gives the largest $w$, because of the large region of $r *$ with low $w$ which occurs in the cases with small $\mathrm{T}_{\mathrm{c}} / \mathrm{T}_{\mathrm{p}}$ (see Fig $4 \mathrm{~b}$ ). Also, $\bar{w} / \mathrm{w}_{\mathrm{m}}$ decreases as $\mathrm{M}_{\mathrm{tm}}$ increases, because of the more rapid variation of $\mathrm{w}$. with $\mathrm{r}^{*}$ as $\mathrm{M}_{\mathrm{tm}}$ increases. The cases with $g_{m}<g_{m}$ (crit) have lower values of $\bar{w} / w_{m}$ than those with $g_{m}>g_{m}$ (crit) because the former do not have the extended region of high $w$ which occurs in the latter near the periphery.

The density ratio depends principally on the temperature ratio, as may be seen from Fig 20. As $\mathrm{T}_{\mathrm{p}} / \mathrm{T}_{\mathrm{c}}$ decreases, the average density in the tube decreases compared to that at the periphery. There is also a small effect of $\mathrm{M}_{\mathrm{tm}}$ and $\mathrm{w}_{\mathrm{m}}$, which is due to the fact that increasing either of these decreases $\mathrm{p}_{\mathrm{Oc}} / \mathrm{p}_{\mathrm{Op}}$, hence lowers the average density compared to that at the periphery.

The final nuclear coupling parameter is $g_{p}$, which is a measure of the neutron track length required to give the heat addition rate implied by the specified value of $\mathrm{g}_{\mathrm{m}}$. It depends very strongly on the temperature ratio, and somewhat less on $\mathrm{M}_{t m}$ and $\mathrm{w}_{\mathrm{m}}$, as may be seen from Fig 2l. The actual requirements placed on the neutron track length $\phi$ (see Eq 40) are somewhat masked by the fact that $g_{p}$ is also proportional to $n_{2 p} / T_{p}$. As $T_{p} / T_{c}$ is reduced, for a given value of $T_{c}$, $\hat{n}_{2 p}$ increases very rapidly if $T_{c} / T_{p}$ is $\frac{p}{s}$ il (see 
Fig $6 \mathrm{~b})$. Thus, $\phi$ does not increase as rapidly as it appears to from Fig 21 . Again, the reason $g_{p}$ for the systems with $g_{m}<g_{m}$ (crit) falls far below that for the systems with $g_{m}>g_{m}$ (crit) is that $n_{2 p}$ for the former is much less than that for the latter (see Fig 6). Representative numerical values for $\varnothing$ will be given later in examples.

Discussion of the Principal Assumptions

and Proposal for Experimental Verification

The two most important assumptions involved in the preceding analysis of the vortex heating -separation process are that the flow in the tube is laminar and that it is inviscid. Of these, the most critical is the first. It will be shown that if the flow is laminar, the neglect of viscous effects is probably not serious; however, if the flow is turbulent, the effect of the turbulent mixing on the separation process cannot be predicted at present.

The purposes of the present section are: (1) to present an argument which indicates that it is not obvious that the flow will be turbulent; (2) to give an estimate of the viscous effects for laminar flow; and (3) to propose a series of experiments designed to check the assumptions in a logical order.

Stability of the flow in the vortex tube:

The gas flow in the vortex tube is of a type with which there is no previous experience, and there seems to be no sensible criterion based on Reynold's number which will give an indication as to whether the flow will be laminar or turbulent. Since the propellant must be introduced through small jets, as in Fig l, it seems, intuitively, at first sight that the flow will in all probability be turbulent.

However, the heating effect in the tubes should tend to stabilize the flow, that is, prevent its becoming turbulent. This may be seen by remembering first that the transition from laminar flow to turbulent flow occurs when inertial forces in the fluid become large enough so that a random fluctuating motion can exist despite the dissipative, or damping, effect of viscous forces. In fact, the Reynold 's number has been characterized as the ratio of inertial to viscous forces. Now in the vortex tube there is an additional stabilizing force which will help to prevent the formation of random fluctuations, at least in the radial direction. This is the body force which results from the vortex field and the temperature gradient. In order to move a small element of fluid instantaneously from some radius to another radius where the temperature is lower and the density higher, a buoyant force must be overcome, which may aid 
in suppressing turbulence. This effect, or its inverse, has been termed "Taylor instability". (6) The general principle is that the interface between two fluids of different densities is destabilized by an acceleration toward the denser fluid, and stabilized by an acceleration toward the less dense fluid. In the present case, there is an extremely large acceleration toward the center of the tube (of the order of $10^{6}$ to . $10^{8}$ times the acceleration of gravity). It therefore seems quite possible that the flow may be laminar in the cases where the temperature increases rapidly toward the center of the tube.

\section{Estimate of viscous effects:}

It is clear that viscous shear forces at the periphery of the vortex tube will tend to cause the actual tangential velocity, and hence the actual vortex field strength, to be less than that predicted by the preceding calculations, which assume that viscous effects are negligible. In order to estimate this effect, it will be assumed, as in the separation calculation, that the flow is laminar. It will be further assumed that the entering fluid is introduced uniformly over a cylindrical surface which has a dimensionless radius $r^{*}=a$, where $a<r$, as shown in Fig 22 .

The equatipp expressing conservation of angular momentum of the fluid may be written $(4)$,

$$
\frac{d v_{0}}{d r}+\frac{v_{0}}{r}=\frac{\mu}{\rho_{0} u_{0}}\left\{\frac{d^{2} v_{0}}{d r^{2}}+\frac{1}{r} \frac{d v_{0}}{d r}-\frac{v_{0}}{r^{2}}\right\}
$$

where the notation is the same as has been used previously.

This equation may be made dimensionless by dividing $v_{0}$ by the tangential velocity of the entering jet, $v_{j}$, and dividing $r$ by $r_{m} \cdot$ Thus,

$$
\frac{d\left(v_{0} / v_{j}\right)}{d r^{*}}+\frac{\left(v_{0} / v_{j}\right)}{r^{*}}=\frac{\mu}{\rho_{0} u_{0} r_{m}}\left\{\frac{d^{2}\left(v_{0} / v_{j}\right)}{d r^{* 2}}+\frac{1}{r^{*}} \frac{d\left(v_{0} / v_{j}\right)}{d r^{*}}-\frac{\left(v_{0} / v_{j}\right)}{r^{* 2}}\right\}
$$


Now $\rho_{0} u_{0} r_{m}=\rho_{0 m} u_{0 m}{ }^{r} \rho_{0}^{*} u_{0}^{*}=-\frac{m_{1}}{2 \pi}\left(1+w_{c}\right) \frac{1}{r^{*}} \cdot$

and Eq (46) finally becomes,

$$
\frac{d\left(v_{0} / v_{j}\right)}{d r^{*}}+\frac{v_{0} / v_{j}}{r^{*}}=-\frac{2 \pi \mu}{m_{1}\left(1+w_{c}\right)^{*}}\left\{\frac{d^{2}\left(v_{0} / v_{j}\right)}{d r^{* 2}}+\frac{1}{r^{*}} \frac{d\left(v_{0} / v_{j}\right)}{d r^{*}}-\frac{\left(v_{0} / v_{j}\right)}{r^{* 2}}\right\}
$$

It can be seen immediately that the parameter which measures the effect of viscosity on the system is $M_{I} / 2 \pi \mu$. If it is large, then we have,

$$
\frac{a\left(v_{0} / v_{j}\right)}{d r^{*}}+\frac{\left(v_{0} / v_{j}\right)}{r^{*}} \approx 0
$$

or $v_{0} r=$ const. This means that the angular momentum of the fluid is conserved as it moves radially. On the other hand, if $M_{1} / 2 \pi \mu$ is small, we have,

$$
\frac{d^{2}\left(v_{0} / v_{j}\right)}{d r^{* 2}}+\frac{1}{r^{*}} \frac{d\left(v_{0} / v_{j}\right)}{d r^{*}}-\frac{v_{0} / v_{j}}{r^{* 2}} \approx 0
$$

The magnitude of $W_{1}$ is set by the binary diffusion process, as expressed by Eq (42). A formula for the viscosity of a gas mixture is given in Ref (5). Assuming the hard-sphere model for molecular collisions, that $\mathrm{m}_{2}>>\mathrm{m}_{1}$, and that $n_{2}<<n_{1}$, this expression may be reduced to:

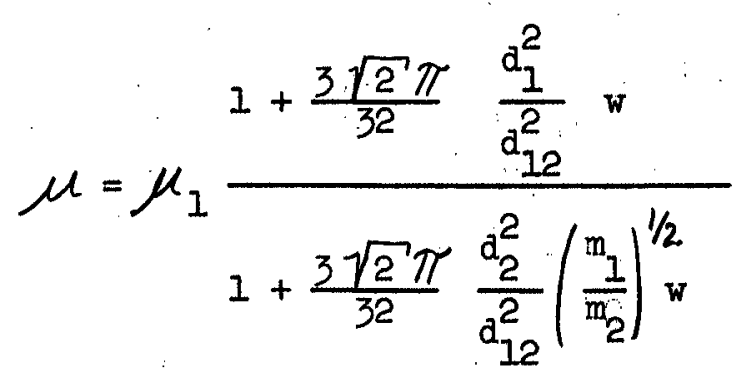


where $\mu_{1}$ is the viscosity of the light gas, $d_{1}$ and $d_{2}$ are the effective hard-sphere collision diameters for light and heavy molecules, and $d_{12}$ is the effective hard-sphere diameter for collisions between light and heavy molecules.

To the same approximation as used in $\mathrm{Eq}(50), \mu_{1}$ is given by,

$$
\mu_{1}=\left(k m_{1} T\right)^{1 / 2} / \pi^{3 / 2} \cdot d_{1}^{2}
$$

Now taking the value of $M_{1}$ given by $\mathrm{Eq}(42), M_{1} / 2 \pi \mu$ may be written,

$\frac{m_{1}}{2 \pi \mu}=0.833 \gamma \frac{d_{1}^{2}}{a_{12}^{2}}\left(\frac{m_{2}}{m_{1}}-1\right) \frac{\Psi}{1-w_{c} / w_{m}}\left(1+\frac{m_{1}}{m_{2}}\right)^{\frac{1}{2}}\left[\frac{1+0.416 \frac{d_{2}^{2}}{d_{12}^{2}}\left(\frac{m_{1}}{m_{2}}\right)^{1 / 2} w_{p}}{1+0.416 \frac{w_{1}^{2}}{d_{12}^{2}} w_{p}}\right]$

Assuming that the quantity in braces is of order unity, i.e. $w_{p}$ is not too large, and that $T_{0}^{*}$ and $r_{0}^{*}$ are of order unity, it is clear that for $\mathbb{H}_{2}$ and $\mathrm{Pu}$ or U gas, $\mathrm{K}_{1} / 2 \mathrm{q} \mu \mathrm{is}$ of order $100 \Psi$. Since $\Psi$ varies between $1 / 4^{2}$ and $I$, $m_{1} / 2 \pi \mu$ varies from about 25 to 100.

Thus Eq (48) is a good approximation to the flow if $r^{*}$ is less than a, the dimensionless radius at which the fluid is introduced, and in this region the flow is essentially inviscid. However, if $r^{*}$ is greater than $a, m_{1}$ is effectively zero, and Eq (49) applies.. In this region, viscous forces are dominant, while in the region of $r *$ less than $a$, inertial forces are dominant. 
The viscous torque tending to retard the vortex flow is therefore due to the shear layer between $r^{*}=a$ and $r^{*}=r_{p^{*}}^{*}$ Its magnitude may be estimated from Eq (49), the solution of which is,

$$
v_{0} / v_{j}=A_{1} r^{*}+A_{2} \frac{1}{r^{*}}
$$

where $A_{1}$ and $A_{2}$ are constants. Clearly $v_{0} / v_{j}=0$ at $r^{*}=r_{p}^{*}$. hence

$$
\frac{v_{0}}{v_{j}}=\frac{v_{0}(a)}{v_{j}} \frac{a}{r_{p}^{* 2}-a^{2}}\left(\frac{r_{p}^{* 2}}{r^{*}}-r^{*}\right)
$$

where $v_{p}(a)$ is the actual tangential velocity at the dimensionless radius, $a$, where the fluid is introduced.

Now $v_{0}(a) / v$ may be determined by equating the torque exerted on the fluid in the tube, by the entering fluid, to the torque caused by shear at the periphery. Thus,

$$
a M_{I_{j}}\left(I-\frac{v_{0}(a)}{v_{j}}\right)=-\left.\mu \frac{d v_{0}}{d r}\right|_{r_{p}} 2 \pi r_{p} r_{p}^{*}
$$

From Eq (53),

$$
\left.\frac{d\left(v_{0} / v_{j}\right)}{d r^{*}}\right|_{r_{p}^{*}}=-\frac{v_{0}(a)}{v_{j}} \frac{2 a}{r_{p}^{* 2}-a^{2}}
$$


Substituting in Eq (54), and solving,

$$
\frac{v_{0}(a)}{v_{j}}=\frac{m_{1} / 2 \pi \mu}{m_{1} / 2 \pi \mu+r_{p}^{* 2} /\left(r_{p}^{* 2}-a^{2}\right)} .
$$

From Eq (55), two points are clear. First, if a approaches $r_{p}^{*}, v_{0}(a) / v_{j}$ becomes small, and the effectiveness of vortex formation is poor. Second, for a given ratio of $\mathrm{r} / \mathrm{a}$, the effectiveness is improved as $m_{1} / 2 \pi \mu$ increases. This is shown in Fig 23 .

For the range of $M_{1} / 2 \pi \mu$ of interest, $i_{\text {.e. from }} 25$ to 100 , the effec. tiveness of vortex formation predicted by this simple model is adequate.

A more general conclusion which can be drawn from the simple analysis is that the effectiveness of vortex formation is determined by the magnitude of $M_{1} / 2 \pi \mu$. This quantity must therefore be considered an important similarity parameter in any experimental study of vortex heating-separation devices.

\section{Suggestions for experimental verification of the analysis:}

As has already been mentioned, the principal questions which require experimental investigation involve the nature of the flow in the tube, and may really be: reduced to one question. The question is whether or not it is possible to create a vortex field of the strength required to achieve separation, with the low mass flow rates which are required by the diffusion process.

Except for the unknown effect of heat addition on the flow stability, this question can be answered by a simple experiment which involves no diffusion. The experiment should be so designed that three similarity requirements are satisfied. First, in order to insure dynamic similarity between the experiment and the actual vortex tube, the inlet Mach numbers should be the same. Second, the Reynold's number, based on some tube dimension, such as its diameter, should be the same. Finally, the mass flow rate per unit of tube length divided by the dynamic viscosity, i.e. $M_{I} / 2 \pi \mu$, should be the same for the experiment and . the actual device. In order to verify the strength of the vortex field it should be sufficient to measure the radial pressure distribution, say at a closed end. 
If the result of this experiment is negative, in the sense that the vortex field is much weaker than is predicted by theory, it must be conm sidered inconclusive, because of the possible stabilizing effect of heat addition. Thus, should the result be negative, it is suggested that the stabilizing effect of a positive density gradient (in the direction of increasing radius) be studied.

At present it appears very difficult to simulate, in an experiment, the volume heating which is expected to produce such a density gradient in the actual vortex reactor. However, there is a possibility of prom ducing a stabilizing density gradient by adding a heavy gas to the light gas in the tube, and choosing the mass flow rate of light gas and its entrance Mach number so that the concentration of heavy gas decreases rapidly from the periphery of the tube toward the center. For example, if, in Fig. 7, the value of $r^{*}$ corresponding to the tube periphery is taken as 0.9 , then for large values of $\mathrm{w}_{\mathrm{m}}$, a considerable favorable density gradient is produced. It is of course necessary that separation of the heavy and light gases be obtained before this stabilizing effect can occur; however, a very heavy gas (for example tetraethyl lead) might be used, to make the separation possible at lower vortex strengths.

If the result of the viscous experiment is favorable, then it is suggested that the next logical step is to attempt to effect the separam tion itself. For this second experiment, some mixture of gases such as hydrogen and mercury vapor, or hydrogen and iodine vapor, might be suitable. A complete formulation of the separation experiment must await the results of the viscous experiment; however, two limitations should be noted.

First, unless a volume heat source can be incorporated into the experiment, the concentration profiles obtainable will be limited to the type shown in Fig. ( $6 a$ ) for $g_{m}<g_{1}(c r i t)$. The more extreme concentration variation shown in Fig. (6b) results from the heating effect.

Second, success in obtaining the type of concentration profile shown in Fig. ( $6 a)$ will not guarantee that the more extreme profiles shown in Fig. (6b) (and the associated high temperature ratios) are also obtainable. Viscous effects may be expected to be most important near the periphery of the tube, just where the most extreme concentration variation occurs for the high temperature ratio cases. Thus, it appears that experimental proof of the feasibility of obtaining temperature ratios greater than about 3 can be obtained only by incorporating a volume heat source into the separation experiment. 
The significance of the results obtained from the preceding analysis. of the heating-separation process may perhaps be better revealed if incorporated into the performance analysis of a vortex-cavity reactor for rocket propulsion. In the present section the characteristic performance parameters of the rocket are calculated in terms of the parameters derived from the separation analysis and from the nuclear configuration. To be definitive, such an analysis would have to include an optimization of the entire system for some mission, and this would imply a detailed study of many aspects of the vehicle configuration; as well as those of the power plant. The intent of the present analysis, however, is much more modest; it is simply to provide a physical feeling for the several parameters which characterize the performance of the vortex tube as applied to rocket propulsion.

To this end, the requirements imposed on the reactor system by the overall heat balance, and by criticality, will be estimated. The weight, thrust-to-weight ratio, neutron $f l u x$ and various other parameters will then be given for some representative examples.

It is assumed in these computations that the reactor core consists of a cylindrical bundle of vortex tubes such as that shown in Fig. I, the diameter being equal to the length, $L$, of an individual tube. Both graphite and beryllium oxide will be considered as moderators. In those cases where the temperature ratio, $\mathrm{T}_{c} / \mathrm{T}_{\mathrm{p}}$, is such that heat must be added to the propellant, by fissionable material, before it enters the vortex tubes, it will be assumed that the fissionable material not contained in the tubes is uniformly dispersed in the moderator. Some reduction in overall size and weight should be possible by concentrating this solid fuel in as small a volume as possible, but this refinement will not be considered here. On the basis of the results of Ref. 7 , the volume fraction of moderator in the core will be taken as 0.4 .

Heat balance:

Since the gas in the vortex tubes is essentially transparent to penetrating radiations, it may be assumed that all radiation originating in the tubes deposits its energy in the moderator. This is equivalent to assuming that some fraction, $\xi$, of the total heat generated by fission in the gas is actually deposited in the gas. Now if the fraction of the total reactor core volume occupied by the gas is $\beta, \phi_{\mathrm{g}}$ and $\phi_{\mathrm{s}}$ are the average neutron fluxes in the gaseous and solid regions, $\bar{n}_{2 s}$ and $\bar{n}_{2 g}$ are the respective mean fissionable material concentrations, and $\sigma_{f s}^{2 g}$ and $\sigma_{f g}$ 
are the fission cross-sections in the gaseous and solid regions, a simple heat balance gives,

$$
\frac{\bar{c}_{p g}}{\bar{c}_{p s}}\left(\frac{T_{c}}{T_{p}}-1\right)=\frac{\beta \xi \bar{n}_{2 g} \sigma_{f g} \phi_{g}}{\beta(1-\xi) \bar{n}_{2 g} \sigma_{f g} \phi_{g}+(1-\beta) \bar{n}_{2 s}{ }_{f s} \phi_{s}}
$$

A's $\bar{n}_{2 s}$ is decreased, $T_{c} / T_{p}$ increases, and a maximum attainable value of $T_{c} / T_{p}$ is reached when $\bar{n}_{2 s}={ }_{0}$. In this case,

$$
\frac{T_{c}}{T_{p}}(\max )=1+\frac{\bar{c}_{p s}}{\bar{c}_{p g}} \frac{\xi}{1-\xi}
$$

Because of dissociation, $\bar{c}_{\mathrm{pg}}$ depends rather strongly on $\mathrm{p}_{\mathrm{oc}}$ and $\mathrm{T}_{\mathrm{C}}$. It increases with increasing $T_{c}$ and decreasing $p_{o c}$. The dependence of $T_{c}(\max ) / T_{p}$ on $p_{o c}$ and $\mathrm{T}_{\mathrm{c}}$ is shown in Fig. 24, for $\xi=0.90$. It should be noted that even though the vortex tube itself is capable of very large temperature ratios, (see Fig. 14) this limitation imposed by the heat deposition from penetrating radiations limits a real system to moderate temperature ratios. This limitation applies, to some extent, to all gas-phase fission heating devices .

\section{Criticality:}

Estimates of the critical size of the reactor have been obtained from two group, two region calculations for a completely reflected cylinder. (The calculations were done on ORACIE using a three group, three region coad, modified for two groups and two regions, and a refilector savings program*.

The reactor core was taken to be a clean homogeneous mixture of moderator, plutonium and hydrogen. The proportions of the latter two were determined from the separation analysis. Fast fission was neglected and the resonance escape probability was taken as unity.

The thickness of the beryllium reflector for each reactor was selected to minimize the combined, core and reflector weight.

* These calculations were done by P. G. Lafyatis and M. L. Nelson. 
The fission cross section of plutonium has a marked resonance at a neutron energy of 0.3 electron volts, which colncides with the mean thermal neutron energy (Maxwell distribution) for a temperature of $2330^{\circ} \mathrm{K}$. Thus, the neutron temperature has an important effect on the critical mass for the systems considered here. It was assumed that the neutron temperature was equal to the moderator temperature, which in turn was taken equal to $\mathrm{T}_{\mathrm{p}}$.

\section{Performance:}

For the present purposes, the performance of the vortex tube reactor may be characterized by the specific impulse, the total thrust, and the ratio of thrust to reactor (core and reflector) weight.

The specific impulse will be taken as,

$$
I_{s p}=\frac{1}{B}\left\{\frac{2 \gamma}{\gamma-1} R_{c}\left[1-\left(\frac{p_{a}}{p_{o c}}\right)^{\frac{\gamma-1}{\gamma}}\right]\right\}^{\frac{1}{2}},
$$

where $g$ is the gravitational constant, and $p_{a}$ is the atmospheric pressure at the exit of the rocket nozzle.

The overall thrust of the critical assembly of vortex tubes is given by:

$$
F=N \operatorname{Lg} m_{I} I_{s p}
$$

where $\mathrm{L}$ is the length of the tubes, $N$ is the number of tubes, and $M_{1}$ is the mass flow per unit of tube length. Now the product $m_{1} L$ is related to the nozzle throat radius by the simple relation,

$$
m_{I}=\pi r_{t}^{2} \rho_{t} \sqrt{\gamma R T_{t}}
$$


while

$$
\rho_{t}=\rho_{I c}\left(1+\frac{\gamma-1}{2}\right)^{\frac{-1}{\gamma-1}}
$$

and

$$
T_{t}=T_{c}\left(1+\frac{\gamma-1}{2}\right)^{-1}
$$

so that

$$
m_{I} I=\pi r_{p}^{2}\left(\frac{r_{c}}{r_{p}}\right)^{2} \cdot P_{I_{c}} \sqrt{\gamma \mathrm{RT}_{c}}\left(1+\frac{\gamma-1}{2}\right)^{\frac{\gamma}{\gamma-1}} \cdot\left(\frac{r_{t}}{r_{c}}\right)^{2}
$$

The quantity $r_{t} / r_{c}$ is the ratio of nozzle throat radius to the radius at which $w$ is equal to $w_{c}$ in the vortex tube. Thus, $r_{t} / r_{c}$ can be assigned any value less than unity. With this expression for $M_{I} L_{\text {, }} \mathrm{Eq}$ (59) becomes,

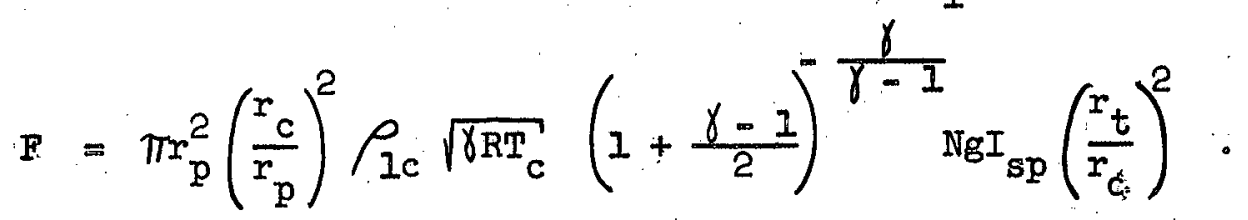

Now $\pi r_{p}^{2} N / \beta$ is the reactor core volume, which equals $\pi L^{3} / 4$ for a square cylinder; hence,

$$
F=\frac{\pi}{4}\left(1+\frac{\gamma-1}{2}\right)^{-\frac{\gamma}{\gamma-1}} \cdot L^{2} \beta \rho_{I c} \sqrt{\gamma R T_{c}}\left(\frac{r_{c}}{r_{p}}\right)^{2} g I_{s p}\left(\frac{r_{t}}{r_{c}}\right)^{2}
$$

Substituting the expression for $I_{\text {sp }}$

$$
F=\frac{\pi}{4} \gamma\left(1+\frac{\gamma-1}{2}\right)^{-\frac{\gamma}{\gamma-1}}\left\{\frac{2}{\gamma-1}\left[1-\left(\frac{p_{a}}{p_{o c}}\right)^{\frac{\gamma-I}{\gamma}}\right]\right\}^{\frac{I}{2}} p_{o c} L^{2} \beta\left(\frac{r_{c}}{r_{p}}\right)^{2}\left(\frac{r_{t}}{r_{c}}\right)^{2}
$$


It can be seen from Eq (63) that the thrust of the vortex reactor is actually limited by the size of the area which can be provided at the back face of the reactor for the exhaust nozzles, without allowing the fissionable material to be blown out. This area is precisely,

$$
\frac{\pi L^{2}}{4} \beta\left(\frac{r_{c}}{r_{p}}\right)^{2}
$$

The thrust is also proportional to the pressure, $p$, but independent of $\mathrm{T}_{c}$. The ratio $r_{c} / r_{p}$ is of course determined by the diffusion

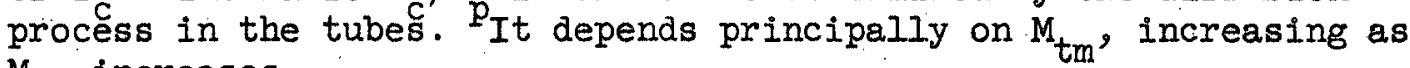
$\mathrm{M}_{\mathrm{tm}}$ increases.

Equation (63) contains the factor $\left(\dot{r}_{t} / r_{c}\right)^{2}$, which may have any value from zero to unity. Thus, for a given set of vortex tube parameters and critical size, the thrust may have any value from zero to that given by $\mathrm{Eq}$ (63) for $r_{t} / r_{c}=1$. As $r_{t} / r_{c}$ is increased, the number of tubes in the reactor is increased, each tube decreasing in diameter. This may be seen by equating Eqs (59) and (62) and solving for $N$. We get,

$$
N=\frac{\pi}{4}\left(1+\frac{\gamma-1}{2}\right)^{-\frac{\gamma}{\gamma-1}} \frac{\rho_{1 c} \sqrt{\gamma_{R T}}}{m_{1}}\left(\frac{r_{c}}{r_{p}}\right)^{2}\left(\frac{r_{t}}{r_{c}}\right)^{2}
$$

For a given set of vortex tube parameters, $\eta_{1}$ and $r_{c} / r_{p}$ are fixed. Then for fixed $p_{o c}$ and $T_{c}$, the number of tubes is proportional to $\left(r_{t} / r_{c}\right)^{2}$.

The neutron track length required to give the heat release rates implied by these performance estimates may be determined from Eq (40), which gives,

$$
\phi=g_{p} \frac{\left(1+w_{c}\right) c_{p l g^{T}} p}{2 n_{2 p} f^{Q}} \quad\left(\frac{m_{q}}{\pi r_{p}^{2}}\right) .
$$

Using Eq (6I) to replace $m_{1} / r_{p}^{2}$,

$$
\phi=g_{p} \frac{\left(1+w_{c}\right) \bar{c}_{p l g} g^{T} p}{2 n_{2 p} \sigma_{f}^{Q}}\left(\frac{r_{c}}{r_{p}}\right)^{2} \cdot \frac{\rho_{1 c} \sqrt{\gamma R T}}{I}\left(1+\frac{\gamma-1}{2}\right)^{-\frac{\gamma}{\gamma-1}} \cdot\left(\frac{r_{t}}{r_{c}}\right)^{2}
$$


Now

$$
n_{2 p}=w_{p} \rho_{I p} / m_{2}=w_{p} \frac{p_{o c}}{m_{2} R T_{c}}\left(\frac{p_{o p}}{p_{o c}}\right)\left(\frac{T_{c}}{T_{p}}\right)
$$

hence

$$
\phi=g_{p} \frac{\left(I+w_{c}\right) \bar{c}_{p l g_{c}} \sqrt{\gamma R T} m_{2}}{2 w_{p}{ }_{p} \sigma_{f}^{Q}}\left(\frac{T_{p}}{T_{c}}\right)^{2}\left(\frac{r_{c}}{r_{p}}\right)^{2}\left(\frac{p_{o c}}{p_{o p}}\right)\left(1+\frac{\gamma-1}{2}\right)^{-\frac{\gamma}{\gamma-1}} \cdot\left(\frac{r_{t}}{r_{c}}\right)^{2}
$$

It can be seen from Eq. (4I) that $g_{p}$ is roughly proportional to $\left(T_{c} / T_{p}\right)^{2}$, so $\phi$ is essentially proportional to $\bar{c}_{p} p^{T_{c}} 3 / 2\left(r_{t} / r_{c}\right)^{2}$. Since $\bar{c}_{p l g}$ increases as $\mathrm{T}_{\mathrm{c}}$ increases, $\phi$ increases quite rapidly with $\mathrm{T}_{\mathrm{c}}$. The proportionality to $\left(r_{t} / r_{c}\right)^{2}$ simply reflects the fact that the total propellant mass flow rate is proportional to $\left(r_{t} / r_{c}\right)^{2}$.

\section{Numerical examples:}

It has been mentioned previously that the temperature ratio, $T_{c} / T_{p}$, is the most important parameter in determining the characteristics of the vortex tube. On the other hand the fissionable material concentration determines the overali size and weight of the reactor. Accordingly, examples have been selected with two representative values of $\mathrm{T}_{c} / \mathrm{T}_{\mathrm{p}}$, and three values of $\mathrm{w}_{\mathrm{m}}$. The exit pressure has been taken as 100 atmospheres for all cases, so $w_{m}$ is nearly a direct measure of the fuel concentration in the gas phase.

Cases 1,2 , and 3 have $T_{c} / T_{p}=1.56$. This temperature ratio corresponds to about half the heat being added to the propellant by solid fuel elements, and half by the vortex tubes. The temperature leaving the solid reactor, ${ }_{\mathrm{p}}$; has been set at the upper. Iimit for graphite fuel elements; namely $4500 \%$; hence, the chamber temperature, $\mathrm{T}_{\mathrm{C}^{\circ}}$ is $7020^{\circ} \mathrm{R}$. This is of the same order as the chamber temperature for contemporary chemical rocket motors (eg. liquid $\mathrm{O}_{2}$ and $\mathrm{JP}_{\mathrm{m}} 4$ give $\mathrm{T}_{\mathrm{c}} \approx 6300^{\circ} \mathrm{R}$ ).

Cases 4, 5, and 6 have the largest values of $T_{c} / T_{p}$ possible for $T_{c}=10,000^{\circ}$. They represent systems with chamber temperatures considerably higher than the best obtainable with chemical rockets. At the same time, the temperatures entering the vortex tubes are considerably below the limits for graphite or the refractory metals. These systems have all fissionable material in the gas phase. 
Some characteristics of these reactors are shown in Table $I$. The values for beryllium oxide moderated reactors are shown in parentheses. The first few quantities listed are characteristic of the vortex tube itself, hence are independent of the moderator used.

Although the reactor weights (core plus reflector) are very high for fuel concentrations of the order of $0.5 \times 10^{18} \mathrm{~cm}^{-3}$, they are quite reasonable if the concentration can be increased to about $5.0 \times 1018 \mathrm{~cm}^{-3}$. These higher fuel concentrations imply high pumping pressures, however. For case 3, pop is 498 atmospheres or 7320 psi. The pumping pressure must be taken as about twice this figure, to allow for the pressure drops in the inlet nozzles and solid reactor. It should be noted that the increase in average fuel concentration, $\bar{n}_{2}$, by a factor of 10 from case 1 to case 3 requires only a little more than doubling of the pumping pressure. This increase is due to the higher pressure drop caused by increasing $w_{m}$. To achieve the same concentration increase by raising the general pressure level with constant $w_{m}$, it would be necessary to increase the pumping pressure tenfold, to about 4000 atmospheres. The beryllium oxide moderated reactors are in all cases lighter than the corresponding graphite moderated reactors. This is due to the smaller neutron slowing down and diffusion lengths in beryllium oxide as compared to graphite.

Although the reactor weights are rather high, the thrust-to-weight ratios are also quite high if $r_{t} / r_{c}$ is near unity. It may, however, be more realistic to take $r_{t} / r_{c}$ a little less than unity, to allow for three dimensional flow effects in the long, thin, vortex tubes. If $r_{t} / r_{c}$ is taken as 0.5 , for example, the beryllium oxide moderated reactor described in case 3 has a thrust of 325,000 Ibs.; and a thrust-to-weight ratio of 61. The latter figure is somewhat lower than that for a chemical system; however, the specific impulse is at least twice that of the best chemical rockets. In order to give this performance the reactor would contain 3,300 vortex tubes, each $0.68 \mathrm{in.}$ in diameter. The average neutron flux would be $0.65 \times 1017$ neutrons $/ \mathrm{sec} \mathrm{cm}^{2}$.

It seems from comparison of cases 3 and 6 , for example, that as $T_{c}$ is increased, the critical size, and weight, of the reactor increase. This effect is due to a lower average fuel concentration in the higher temperature reactor. The gas-phase fuel concentrations, $\bar{n}_{2 g}$, are about the same in the two cases, but the lower - temperature reactor contains considerable solid fuel.

Finally, it must be emphasized again that these results are only exemplary. It is obvious that reactor weight is a very important parameter in these systems; therefore, a detailed criticality analysis must be made before definite conclusions can be drawn as to the advisability of further development of the vortex tube for rocket propulsion. 


\section{Case}

\section{Number}

\begin{tabular}{|c|c|c|c|c|c|c|}
\hline Parameter & 1 & 2 & 3 & 4 & $5^{\circ}$ & 6 \\
\hline$T_{c} / T_{p}$ & 1.56 & 1.56 & 1.56 & 4.05 & 4.05 & 4.05 \\
\hline$\overline{\mathrm{w}_{\mathrm{m}}}$ & 0.5 & 1.0 & 4.0 & 0.5 & 1.0 & 4.0 \\
\hline $\mathrm{M}_{\mathrm{tp}}$ & 1.0 & 1.0 & 1.0 & 1.0 & 1.0 & 1.0 \\
\hline $\mathrm{T}_{\mathrm{p}}, \mathrm{O}_{\mathrm{R}}$ & 7,020 & 7,020 & 7,020 & 10,000 & 10,000 & 10,000 \\
\hline $\mathrm{T}_{p}, \mathrm{O}_{\mathrm{R}}$ & 4,500 & 4,500 & 4,500 & $2 ; 470$ & 2,470 & 2,470 \\
\hline poc, atm & 100 & 100 & 100 & 100 & 100 & 100 \\
\hline$p_{o p}$ atm & 213 & 231 & 498 & 271 & 188 & 338 \\
\hline$r_{c} / r_{p}$ & 0.66 & 0.66 & 0.65 & 0.59 & 0.59 & 0.58 \\
\hline$\overline{\bar{w}} / \mathrm{w}_{\mathrm{m}}$ & 0.34 & 0.35 & 0.36 & 0.56 & 0.56 & 0.54 \\
\hline$g_{p}$ & 0.96 & 0.96 & 1.50 & 56 & 56 & 81 \\
\hline$\eta_{1} 1 \mathrm{~b} / \mathrm{sec} \mathrm{ft}$ & 0.0198 & 0.0198 & 0.0198 & 0.0121 & 0.0121 & 0.0121 \\
\hline$m_{1} / 2 \pi \mu$ & 151 & 149 & 135 & 108. & 95 & 57 \\
\hline$I_{s p}, \sec \left(p_{a}=0\right)$ & 1196 & 1196 & 1196 & 1426 & 1426 & 1426 \\
\hline $\bar{n}_{2 g^{\prime}} \mathrm{cm}^{-3}$ & $0.40 \times 10^{18}$ & $0.85 \times 10^{18}$ & $5.2 \times 10^{18}$ & $0.49 \times 10^{18}$ & $1.01 \times 10^{18}$ & $5.12 \times 10^{18}$ \\
\hline$I$, ft & $\begin{array}{l}15.4 \\
(14.0) \\
\end{array}$ & $\begin{array}{l}10.2 \\
(8.5) \\
\end{array}$ & $\begin{array}{l}3.8 \\
(4.1) \\
\end{array}$ & $\begin{array}{l}16.8 \\
(16.7) \\
\end{array}$ & $\begin{array}{l}11.7 \\
(10.1) \\
\end{array}$ & $\begin{array}{l}4.5 \\
(4.5) \\
\end{array}$ \\
\hline$F /\left(r_{t} / r_{c}\right)^{2}, 1 b$ & $\begin{array}{l}18.7 \times 10^{6} \\
\left(15.4 \times 10^{6}\right) \\
\end{array}$ & $\begin{array}{l}8.2 \times 10^{6} \\
\left(5.7 \times 10^{6}\right) \\
\end{array}$ & $\begin{array}{l}1.1 \times 10^{6} \\
\left(1.3 \times 10^{6}\right)\end{array}$ & $\begin{array}{l}17.7 \times 10^{6} \\
\left(17.5 \times 10^{6}\right) \\
\end{array}$ & $\begin{array}{l}8.6 \times 10^{6} \\
\left(6.4 \times 10^{6}\right)\end{array}$ & $\begin{array}{l}1.2 \times 10^{6} \\
\left(1.2 \times 10^{6}\right)\end{array}$ \\
\hline Weight, Ib & $\begin{array}{l}180,000 \\
(120,000)\end{array}$ & $\begin{array}{l}75,000 \\
(35,000)\end{array}$ & $\begin{array}{l}10,000 \\
(5,300) \\
\end{array}$ & $\begin{array}{l}230,000 \\
(180,000)\end{array}$ & $\begin{array}{l}100,000 \\
(55,000)\end{array}$ & $\begin{array}{l}15,000 \\
(6,700) \\
\end{array}$ \\
\hline $\mathrm{F} /\left(r_{t} / r_{c}\right)^{2} /$ Weight & $\begin{array}{l}104 \\
(129) \\
\end{array}$ & $\begin{array}{l}109 \\
(162) \\
\end{array}$ & $\begin{array}{l}110 \\
(242) \\
\end{array}$ & $\begin{array}{l}77 \\
(97) \\
\end{array}$ & $\begin{array}{l}86 \\
(117)\end{array}$ & (183) \\
\hline $\mathbb{N} /\left(r_{t} / r_{c}\right)^{2}$ & $\begin{array}{l}51,200 \\
(46,500) \\
\end{array}$ & $\begin{array}{l}33,900 \\
(28,200) \\
\end{array}$ & $\begin{array}{l}12,200 \\
(13,200)\end{array}$ & $\begin{array}{l}61,200 \\
(60,800)\end{array}$ & $\begin{array}{l}42,600 \\
(36,800)\end{array}$ & $\begin{array}{l}15,800 \\
(15,800)\end{array}$ \\
\hline$\phi /\left(r_{t} / r_{c}\right)^{2}, \frac{\text { neut }}{\mathrm{cm}^{2} \text { sec }}$ & $\begin{array}{l}0.85 \times 10^{18} \\
\left(0.94 \times 10^{18}\right)\end{array}$ & $\begin{array}{l}0.60 \times 10^{18} \\
\left(0.71 \times 10^{18}\right)\end{array}$ & $\begin{array}{l}0.28 \times 10^{18} \\
\left(0.26 \times 10^{18}\right) \\
\end{array}$ & $\begin{array}{l}1.4 \times 10^{18} \\
\left(1.4 \times 10^{18}\right)\end{array}$ & $\begin{array}{l}0.93 \times 10^{18} \\
\left(1.1 \times 10^{18}\right) \\
\end{array}$ & $\begin{array}{l}0.32 \times 10^{18} \\
\left(0.32 \times 10^{18}\right) \\
\end{array}$ \\
\hline$r_{p}\left(r_{t} / r_{c}\right)$, in. & $\begin{array}{l}0.32 \\
(0.30) \\
\end{array}$ & $\begin{array}{l}0.26 \\
(0.23) \\
\end{array}$ & $\begin{array}{l}0.16 \\
(0.17)\end{array}$ & $\begin{array}{l}0.31 \\
(0.31)\end{array}$ & $\begin{array}{l}0.26 \\
(0.24)\end{array}$ & $\begin{array}{l}0.17 \\
(0.17) \\
\end{array}$ \\
\hline
\end{tabular}

Table 1 - Numerical Examples of Vortex Tube Reactors 


\section{References}

1. Chapman and Cowling, The Mathematical Theory of Non-Uniform Gases, Chapter 8, Cambridge University Press.

2. Chapman and Cowling, The Mathematical Theory of Non-Uniform Gases, p. 237, Cambridge University Press.

3. Chapman and Cowling, The Mathematical Theory of Non-Uniform Gases, p. 245, Cambridge University Press.

4. Lamb, Hydrodynamics, p. 156, Dover.

5. Chapman and Cowling, The Mathematical Theory of Non-Uniform Gases, p. 167, Cambridge University Press.

6. Taylor, G. I., The Instability of Liquid Surfaces When Accelerated in a Direction Perpendicular to their Planes,' Proc. Roy Soc A 201, p. $192-194$ (1950).

7. Martinez, J. S., Krieve, W. F., Mihalczo, J.T, Robinson, J. B., Huffman, F. N., Pengelley, C. D., and Klotz, R. J , "Cavity Reactors for Rocket Propulsion" ORNL CF 57-8-11 (in preparation).

8. R. R. Bate, I. T. Einstein and W. E. Kinney, "Description and Operating Manual for the Three Group, Three Region Reactor Code for ORACLE", ORNL Memorandum CF 55-1-76, January 13, 1955. 


\section{List of Figures and Tables}

\section{Figures:}

1. Schematic diagram of vortex tube with integral exhaust nozzle

2. Behavior of $w / w_{m}$ for $g_{m}$ greater than and less than maximum permissible value

3. Behavior of $\mathrm{w} / \mathrm{w}_{\mathrm{m}}$ for $\mathrm{g}_{\mathrm{m}}$ greater than and less than critical value

4. Variation of density ratio, $w$, with dimensionless radius, $r *$,
a) for $g_{m}$ less than $g_{m}$ (crit)
b) for $g_{m}$ greater than $g_{m}($ crit)

5. Variation of dimensionless temperature, $T *$ with dimensionless radius, $r *$,
a) for $g_{m}$ less than $g_{m}$ (crit)
b) for $g_{m}$ greater than $g_{m}($ crit)

6. Variation of aimensionless fuel concentration, $n_{2}^{*}$, with dimensionless radius, $r^{*}$,
a) for $g_{m}$ less than $g_{m}$ (crit)
b) for $g_{m}$ greater than $g_{m}$ (crit)

7. Effect of the maximum density ratio, $w_{m}$, on, the variation of the density ratio, $w_{3}$ with the dimensionless radius, $r *$

8. Effect of the tangential Mach number at the point of maximum density ratio, $\mathrm{M}_{\mathrm{tm}}$, on the variation of the density ratio, $w$, with the dimensionless radius, $r^{*}$.

9. Dependence of the overall temperature ratio, $T_{p} / T_{c}$, and overall pressure ratio, poc/pops on the choice of the falue, rof of the dimensionless radius which corresponds to the tube periphery,
a) for $g_{m}$ less than $g_{m}($ crit)
b) for $g_{m}$ greater than $g_{m}($ crit). 
10. Dependence of the tangential Mach number at the periphery, $M_{t p}$, on the choice of the value, $r_{p}^{*}$, of the dimensionless radius which corresponds to the tube periphery

a) for $g_{m}$ less than $g_{m}$ (crit)

b) for $g_{m}$ greater than $g_{m}($ crit)

11. Dependence of the relative mass flow capacity, $\Psi$, on the choice of the value, $r_{0}^{*}$, of the dimensionless radius: which corresponds to the tube periphery
a) for $g_{m}$ less than $g_{m}$ (crit)
b) for $g_{m}$ greater than $g_{m}$ (crit)

12. Dependence of the average-to-peripheral propellant density ratio, and the ratio of average to maximum density ratio on the choice of the value, $r^{*}$, of the dimensionless radius which corresponds to the tube periphery
a) for $g_{m}$ less than $g_{m}$ (crit)
b) for $g_{m}$ greater than $g_{m}$ (crit)

13. Dependence of the heating parameter, $g_{p}$ on the value, $r_{p}^{*}$, of the dimensionless radius which corresponds to the tube periphery

a) for $g_{m}$ less than $g_{m}$ (crit)

b) for $g_{m}$ greater than $g_{m}$ (crit)

14. Dependence of the overall temperature ratio, $T_{p} / T_{c}$, on the ratio of the heating parameter, $g_{m}$, to its maximum permissible value
a) for $M_{t m}=0.5$
b) for $\mathrm{M}_{t m}=0.7$
c) for $M_{t m}=1.0$

15. Dependence of the overall pressure ratio, $\mathrm{p}_{\mathrm{oc}} / \mathrm{p}_{\mathrm{op}}$, on the overall temperature ratio, $\mathrm{T}_{\mathrm{p}} / \mathrm{T}_{\mathrm{c}}$
a) for $M_{t m}=0.5$
b) for $M_{t m}=0.7$
c) for $\mathrm{M}_{\mathrm{tm}}=1.0$ 
16. Dependence of the tangential Mach number at the periphery, $\mathrm{M}_{\text {tp }}$, on the overall temperature ratio, $\mathrm{T}_{\mathrm{c}} / \mathrm{T}_{\mathrm{p}}$.

17. Dependence of the relative mass flow capacity; $\Psi$, on the overa 17 temperature ratio, $\mathrm{T}_{\mathrm{p}} / \mathrm{T}_{\mathrm{c}}$.

18. Dependence of the ratio of exit radius to tube radius on the overall temperature ratio, $T_{p} / T_{c}$.

19. Dependence of the ratio of average to maximum density ratio on the overall temperature ratio, $\mathrm{T}_{\mathrm{p}} / \mathrm{T}_{\mathrm{c}}$
a) for $M_{t m}=0.5$
b) for $M_{t m}=0.7$
c) for $M_{t m}=1.0$

20. Dependence of the ratio of average to peripheral propellant densities on the overali temperature ratio, $T_{p} / T_{c}$.
a) for $M_{t m}=0.5$
b) for $M_{t m}=0.7$
c) for $M_{t m}=1.0$

21. Dependence of the heating parameter, $g_{p}$, on the overall temperature ratio, $T_{p} / T_{c}$.

a) for $M_{t m}=0.5$

b) for $M_{t m}=0.7$

c) for $\dot{M}_{t m}=1.0$

22. Introduction of the fluid at a dimensionless radius $a$.

23. Dependence of the ratio of effective to inlet tangential velocities on the fraction of the tube radius at which the fluid enters.

24. Dependence of the maximum obtainable temperature ratio, $\mathrm{T}_{c}(\max ) / \mathrm{T}_{\mathrm{p}}$, (all fissionable material in gas) on exit temperature, $\mathrm{T}_{c}$, and pressure, $\mathrm{p}_{\mathrm{Oc}}$.

Tables:

Table 1 Numerical examples of vortex-tube reactors. 


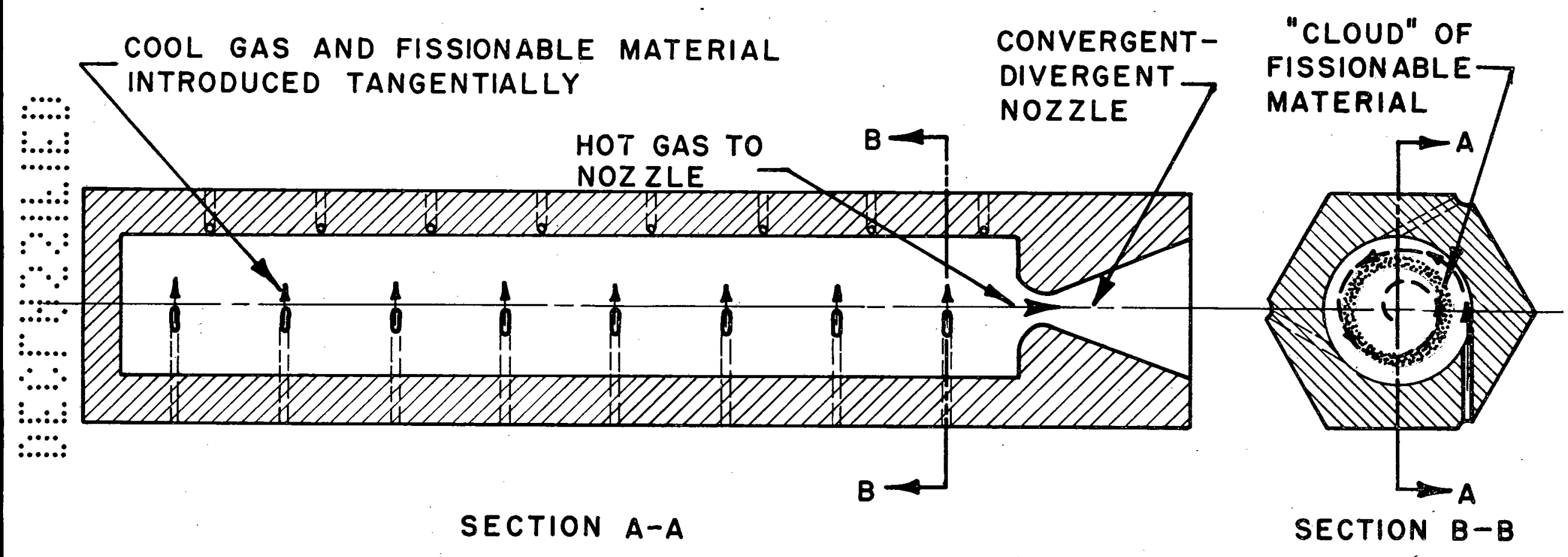

0
8
0
0
0

SPECIALIZATION OF THE VORTEX TUBE FOR ROCKET PROPULSION.

A UNIT VORTEX CELL WITH INTEGRAL NOZZLE

FIGURE 1

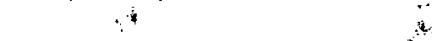



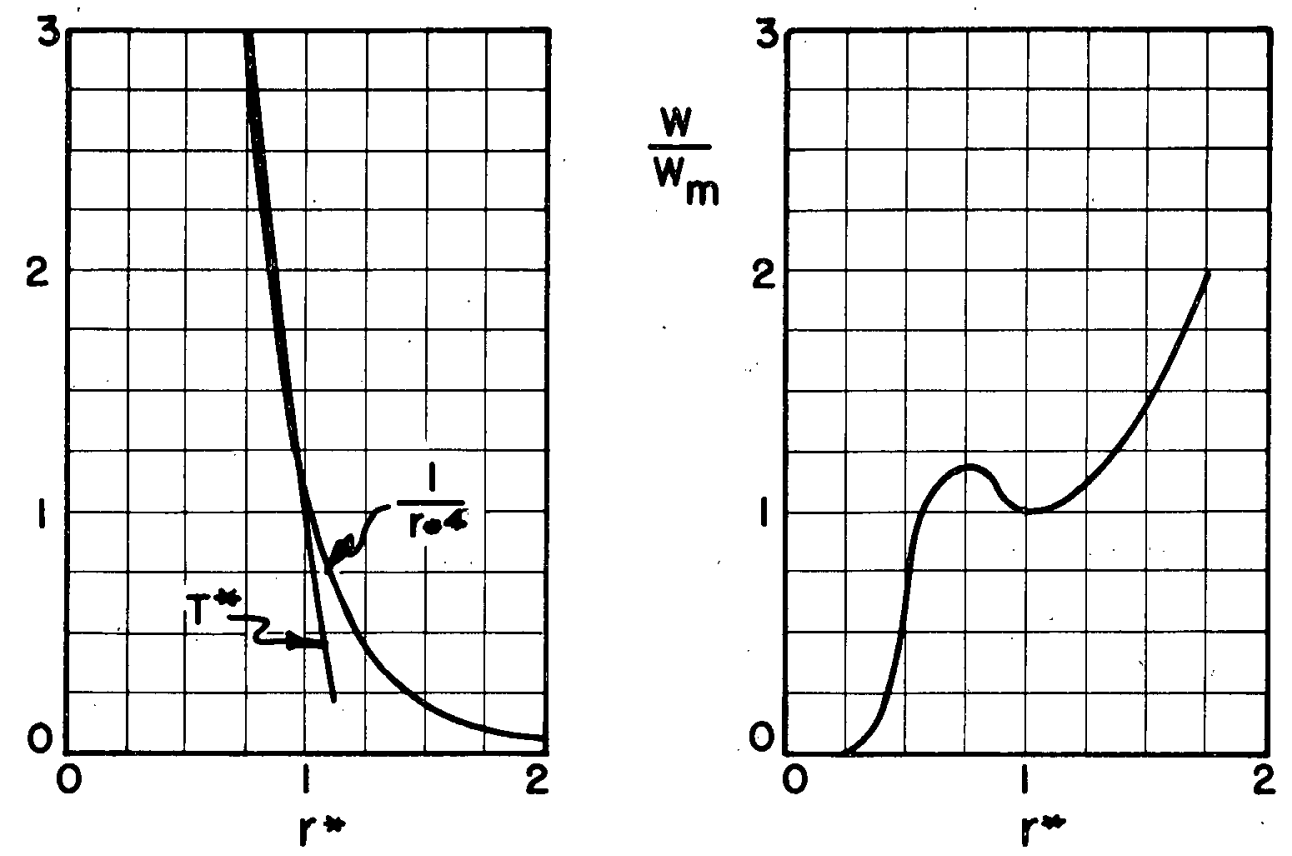

SKETCH (A)
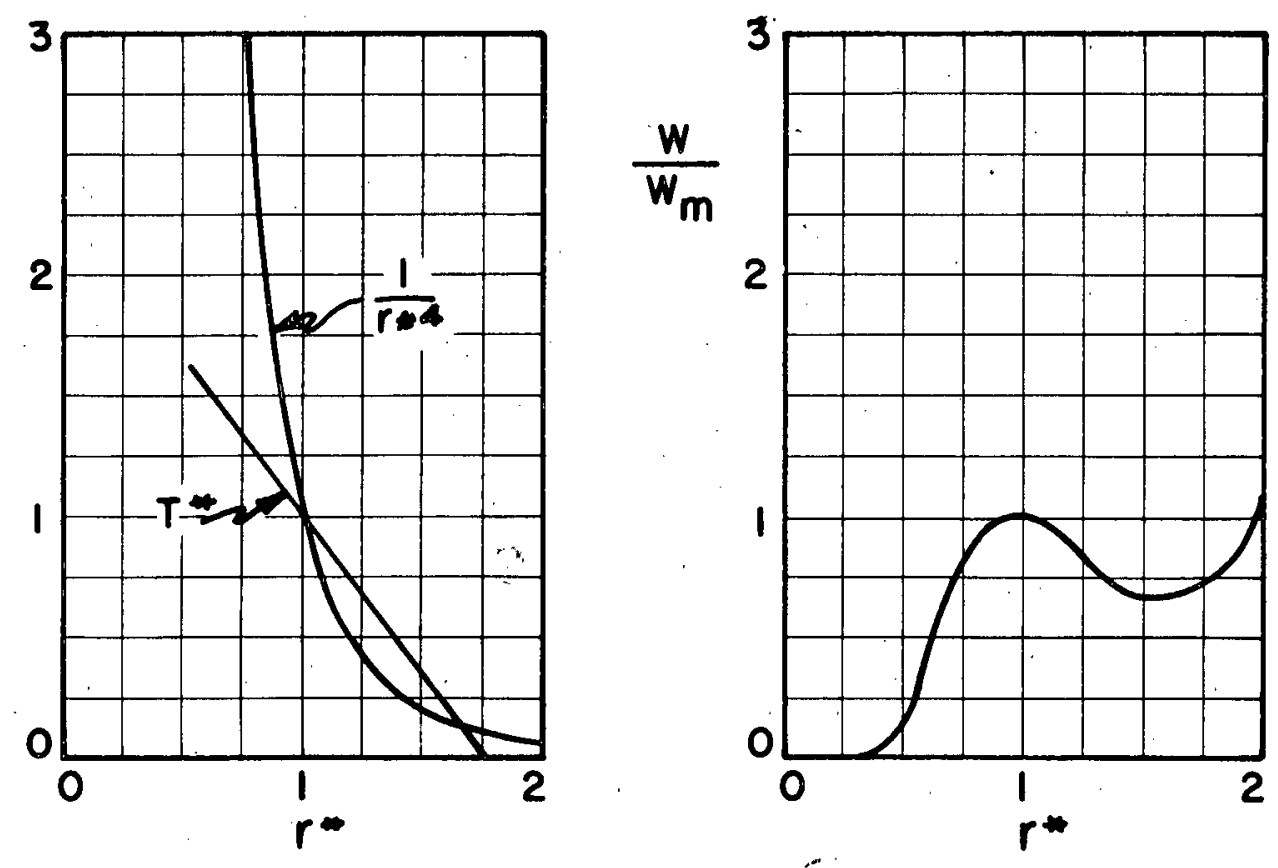

SKETCH (BROO 066

FIG. 2 : BEHAVIOR OF $W / W_{m}$ FOR $g_{m}$ GREATER THAN AND LESS THAN MAXIMUM PERMIS 


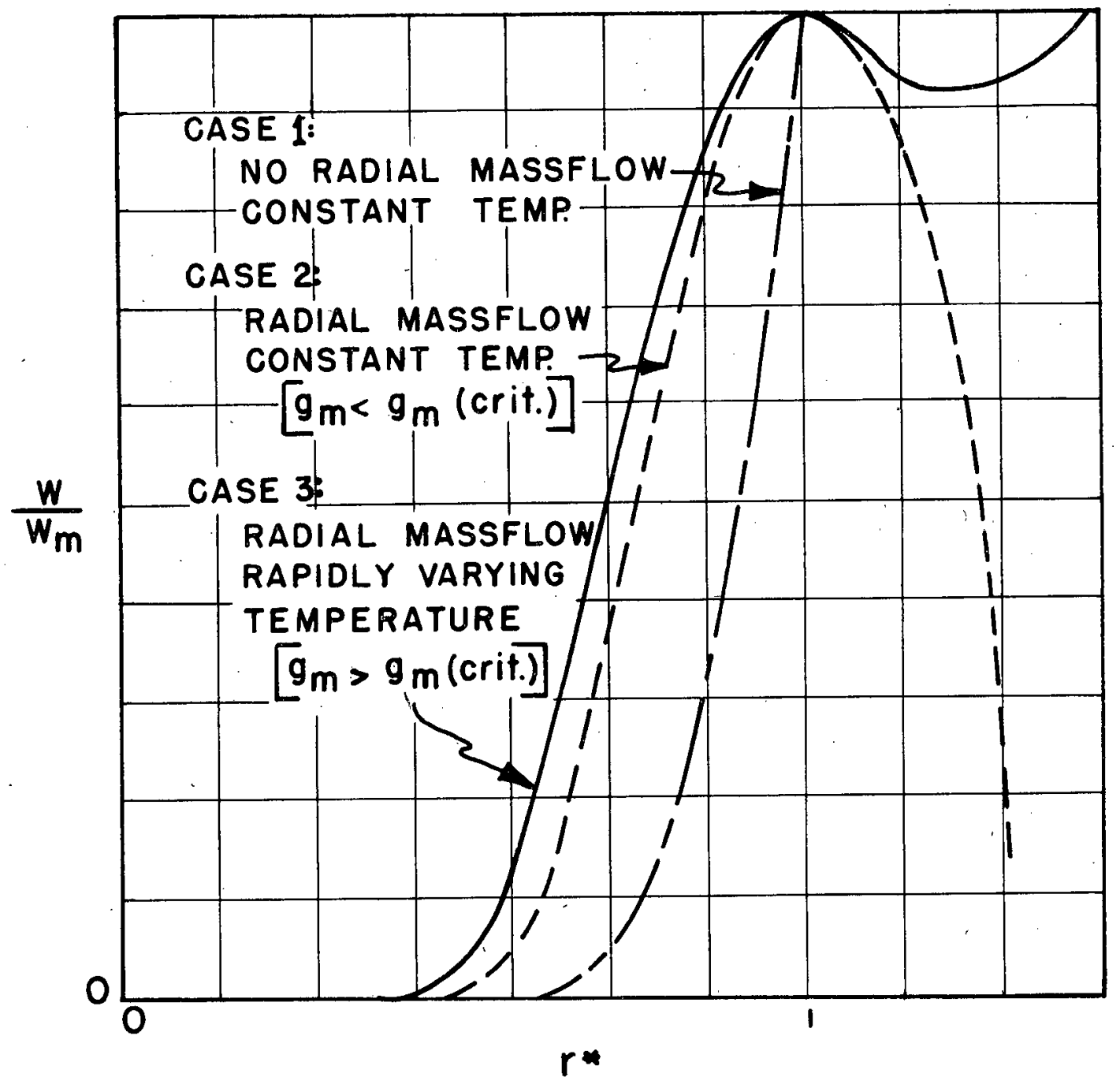

FIG. 3 : BEHAVIOR OF $W / W_{m}$ FOR VARIOUS COMBINATIONS OF HEATING AND RADIAL MASSFLOW.

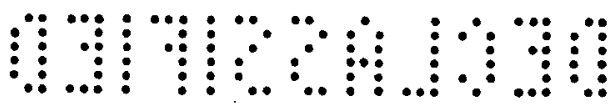



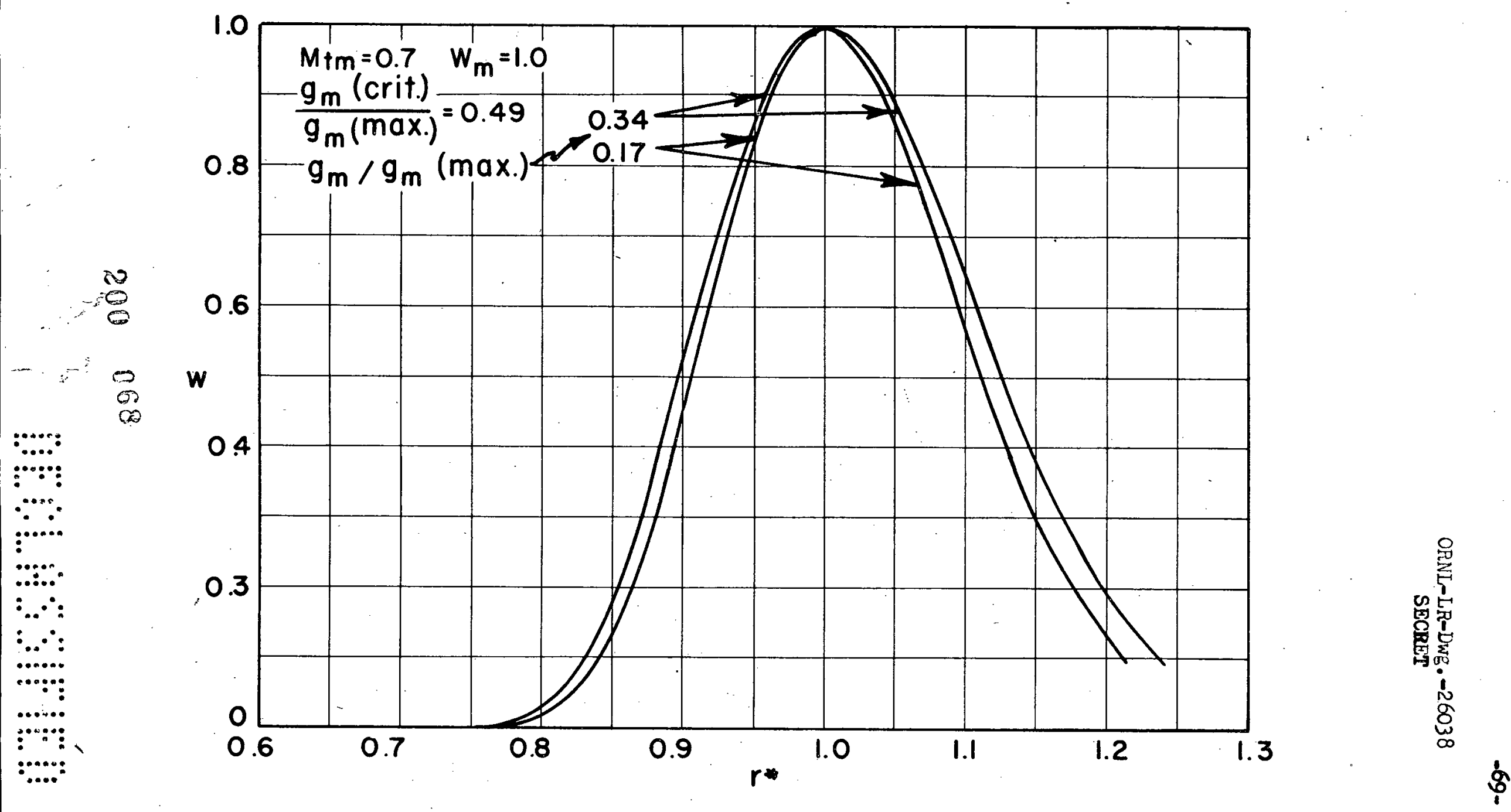

FIG. 4a: VARIATION OF DENSITY RATIO, W, WITH DIMENSIONLESS RADIUS, $r^{*}: F O R \cdot g_{m}$ LESS THAN $g_{m}$ (Crit.) 
$\therefore . . . \cdot$

¿...:!

......

…:

$\because \because$

$\because \because$

$\cdots$

$:$

$\therefore$

$\vdots \ldots$

$\therefore \cdots:$

9
8
8

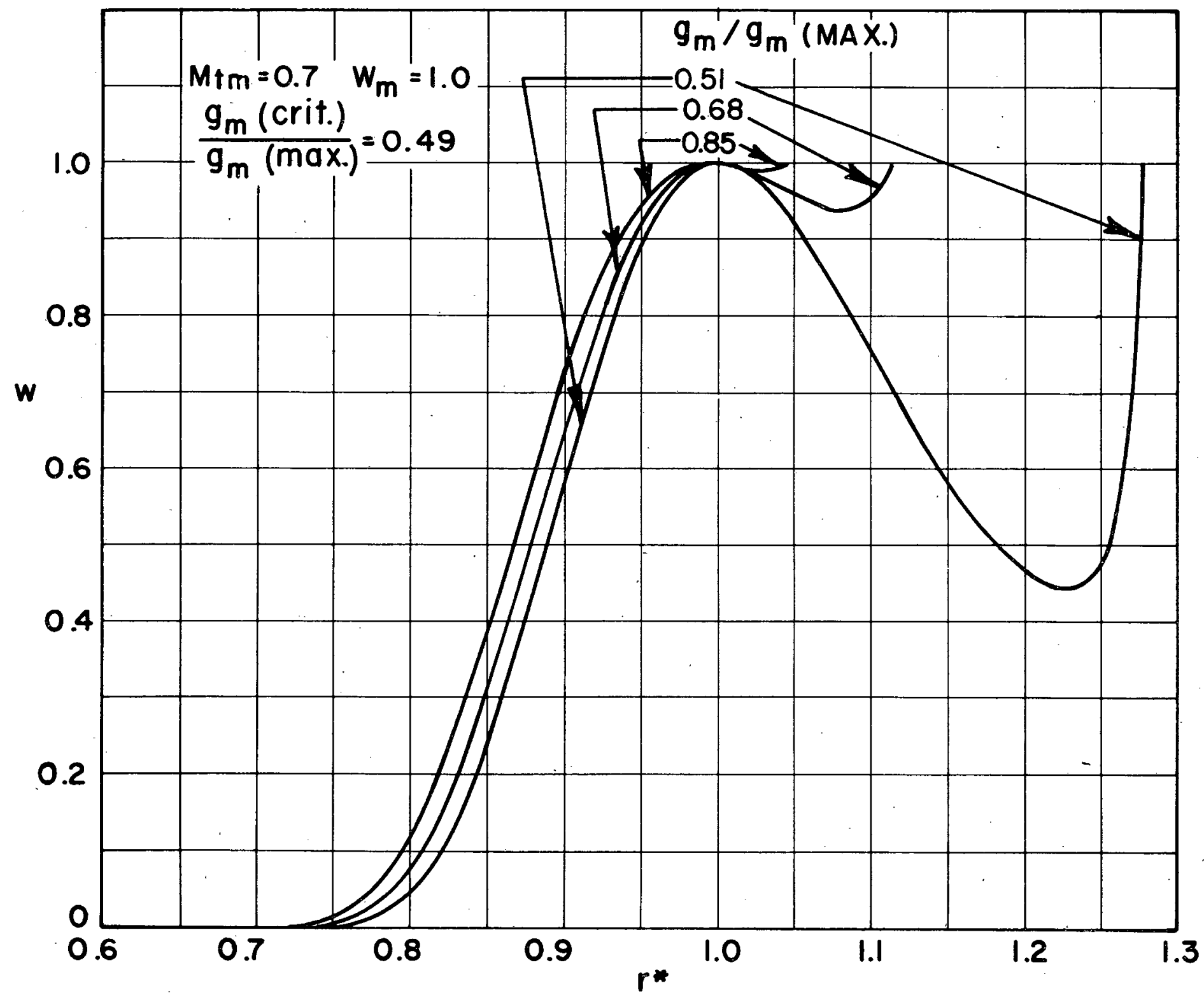

FIG. $4 \mathrm{~b}$ : VARIATION OF DENSITY RATIO, W, WITH DIMENSIONLESS RADIUS, $r^{*}$ : FOR $g_{m}$ GREATER THAN $g_{m}$ (CRIT.) 


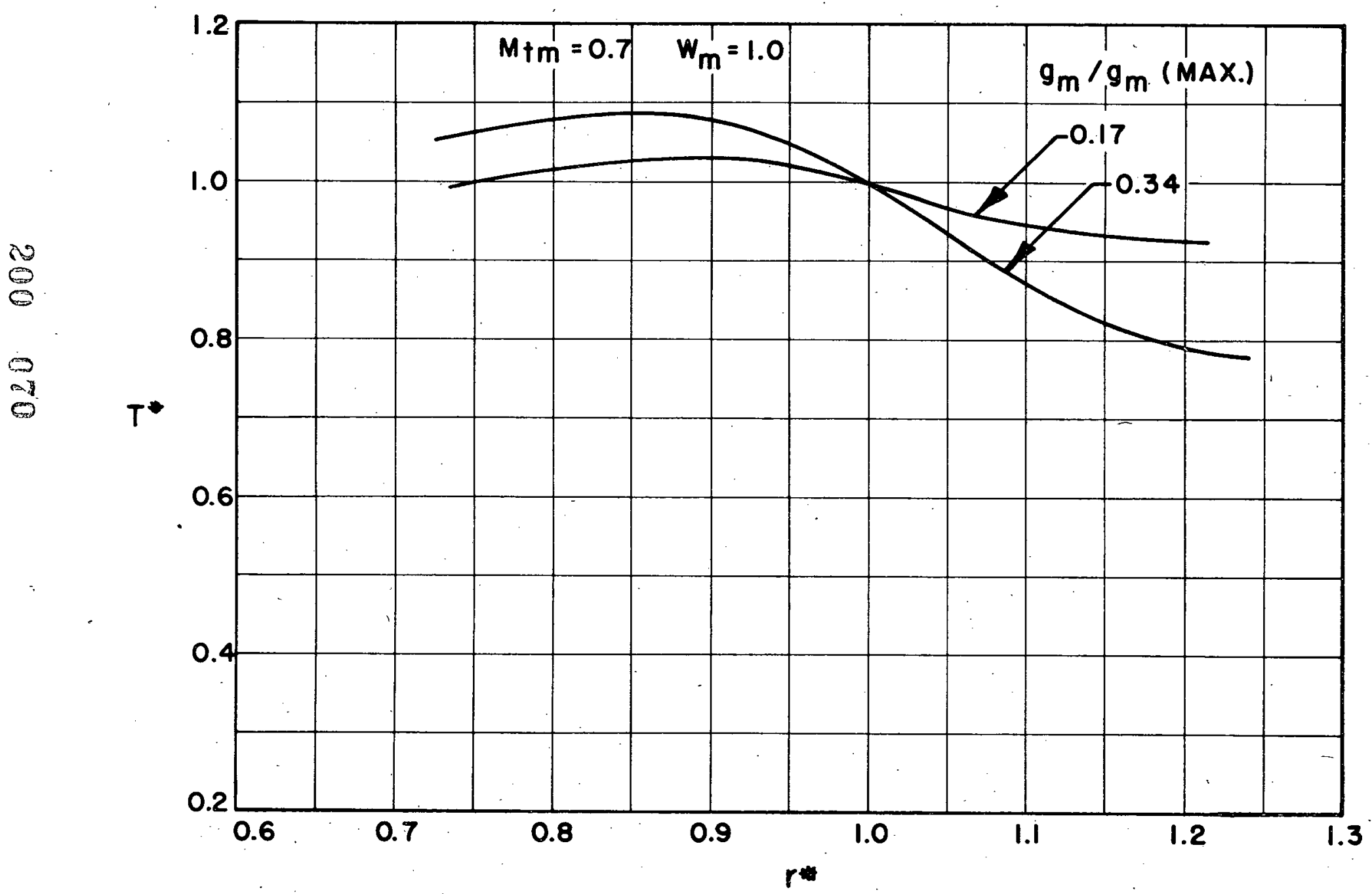

FIG. 5a: VARIATION OF DIMENSIONLESS TEMPERATURE, $T$ * WITH DIMENSIONLESS RADIUS, $r^{*}$ : FOR $g_{m}$ LESS THAN $g_{m}$ (CRIT.) 
$\therefore \cdots:$

$\vdots \ldots:$ :

$\ldots$ :

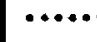

$\because \because$

$\because \because$

…...

$\vdots \ldots$

$\therefore \ldots$

$\vdots . . .: \vdots$

$\therefore$

ne

$\Leftrightarrow$

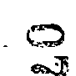

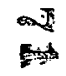

FIG.5b: VARIATION OF DIMENSIONLESS TEMPERATURE, $T^{*}$ WITH DIMENSIONLESS RADIUS, $r^{*}$ : FOR $g_{m}$ GREATER THAN $g_{m}$ (CRIT.) 


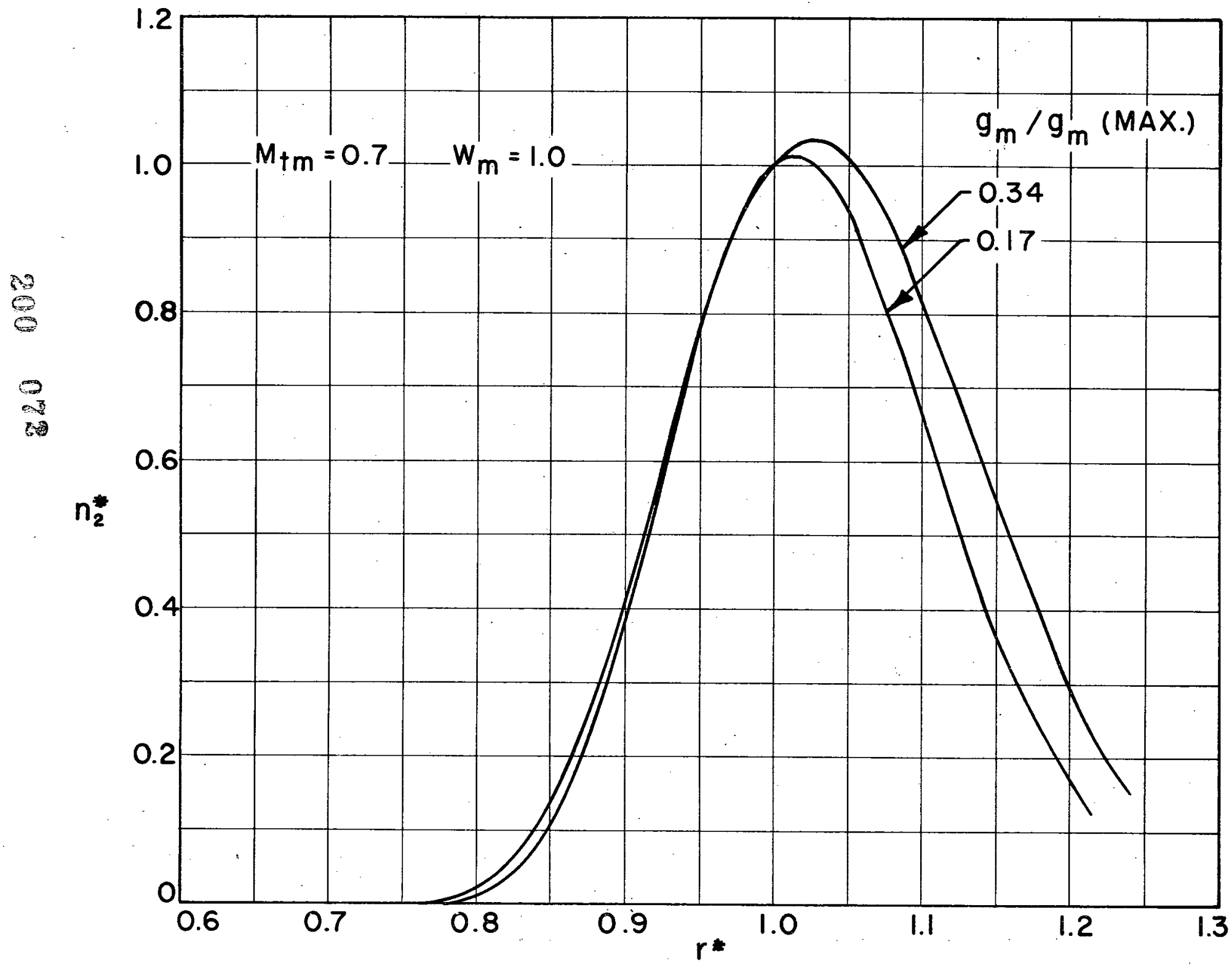

FIG.6a: VARIATION OF DIMENSIONLESS FUEL CONCENTRATION, $n_{2}^{*}$, WITH DIMENSIONLESS RADIUS, $r^{*}$ :FOR $g_{m}$ LESS THAN $g_{m}$ (CRIT.) 
$\therefore: \cdots:$

$\vdots \ldots .$.

....:.:

$\because \cdots$

$\because \because$

$\because \because \cdots$

$\vdots \ldots .$.

$\therefore \therefore$

$\vdots \ldots:$ :

$\therefore \ldots:$

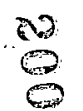

$\infty$

0

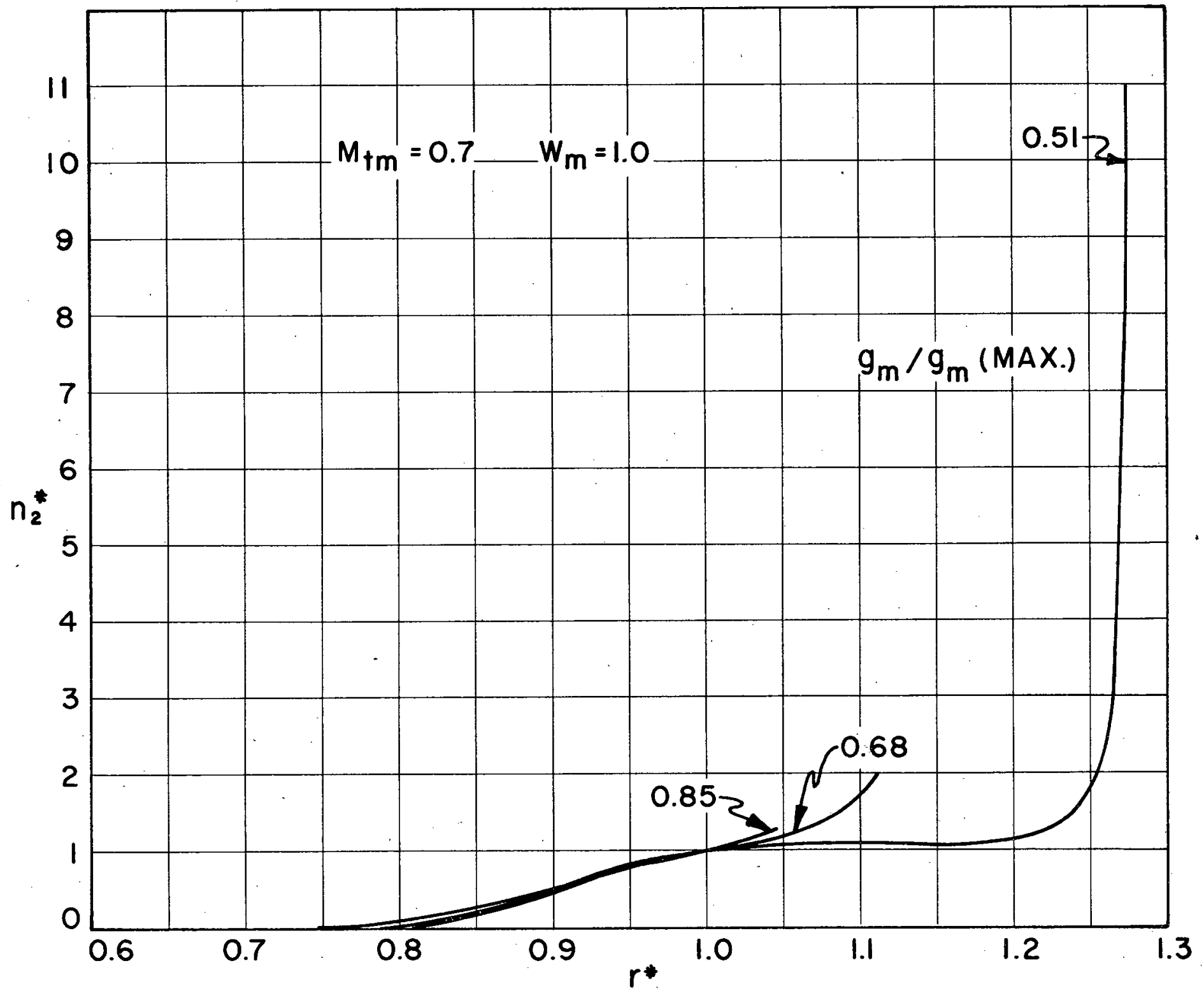

FIG.6b: VARIATION OF DIMENSIONLESS FUEL CONCENTRATION, $\mathrm{n}_{2}^{*}$, WITH DIMENSIONLESS RADIUS, $r$; FOR $g_{m}$ GREATER THAN $g_{m}$ (CRIT.) 


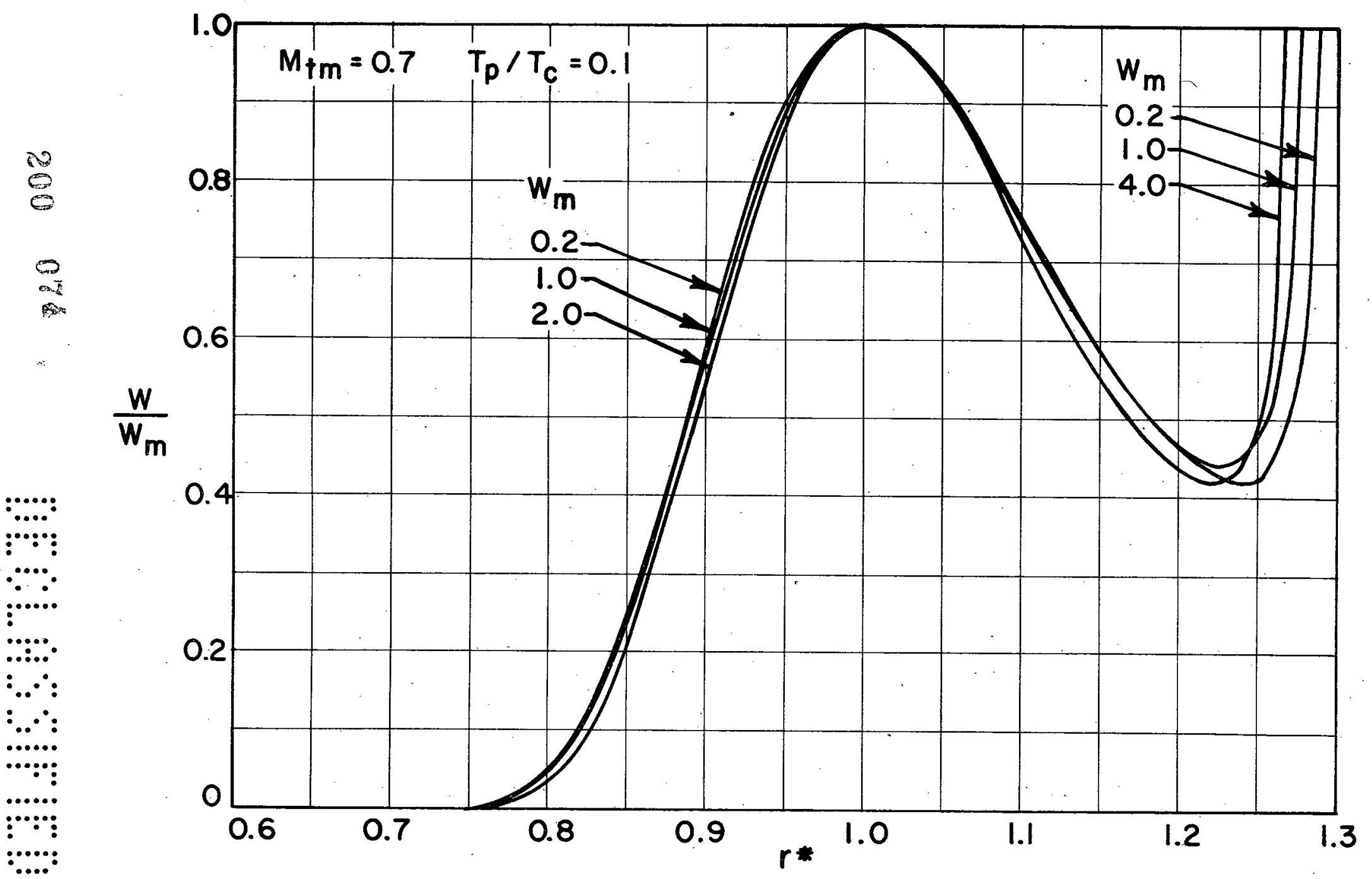

FIG.7: EFFECT OF THE MAXIMUM DENSITY RATIO, $W_{m}$, ON THE VARIATION OF THE DENSITY RATIO, W, WITH THE DIMENSIONLESS RADIUS, $r$ 


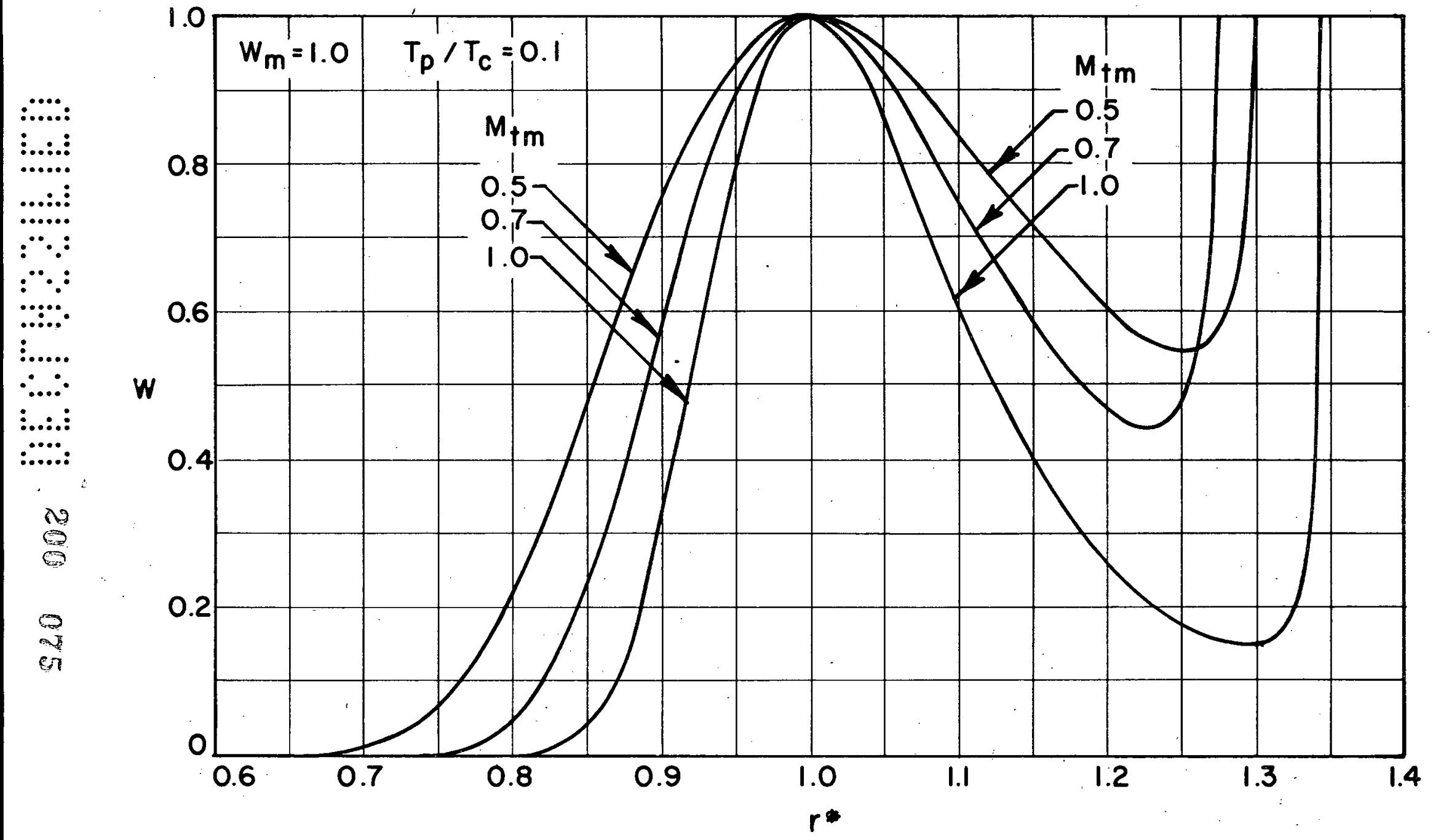

FIG.8: EFFECT OF THE TANGENTIAL MACH NUMBER AT THE POINT OF MAXIMUM DENSITY RATIO, $M_{+m}$, ON THE VARIATION OF THE DENSITY RATIO, $W, W I T H$ DIMENSIONLESS RADIUS, $r^{*}$ 


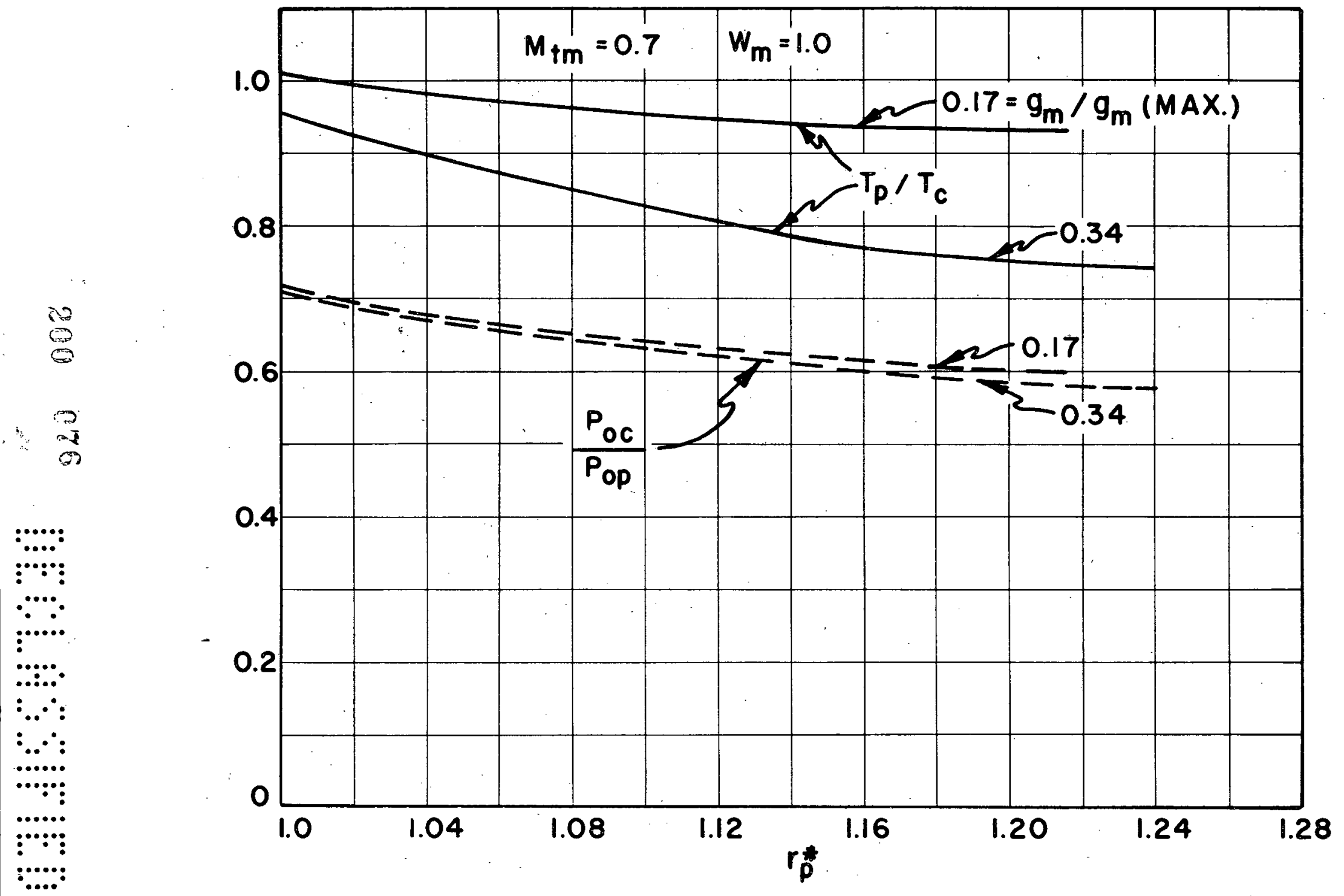

FIG.9a-DEPENDENCE OF THE OVERALL TEMPERATURE RATIO, $T_{p} / T_{c}$, AND OVERALL PRESSURE RATIO, $P_{O C} / P_{O P}$, ON THE CHOICE OF THE VALUE, $r_{p}^{\circ}$, OF THE DIMENSIONLESS RADIUS WHICH CORRESPONDS TO THE TUBE PERIPHERY: FOR $g_{m}$ LESS THAN $g_{m}$ (CRIT.) 


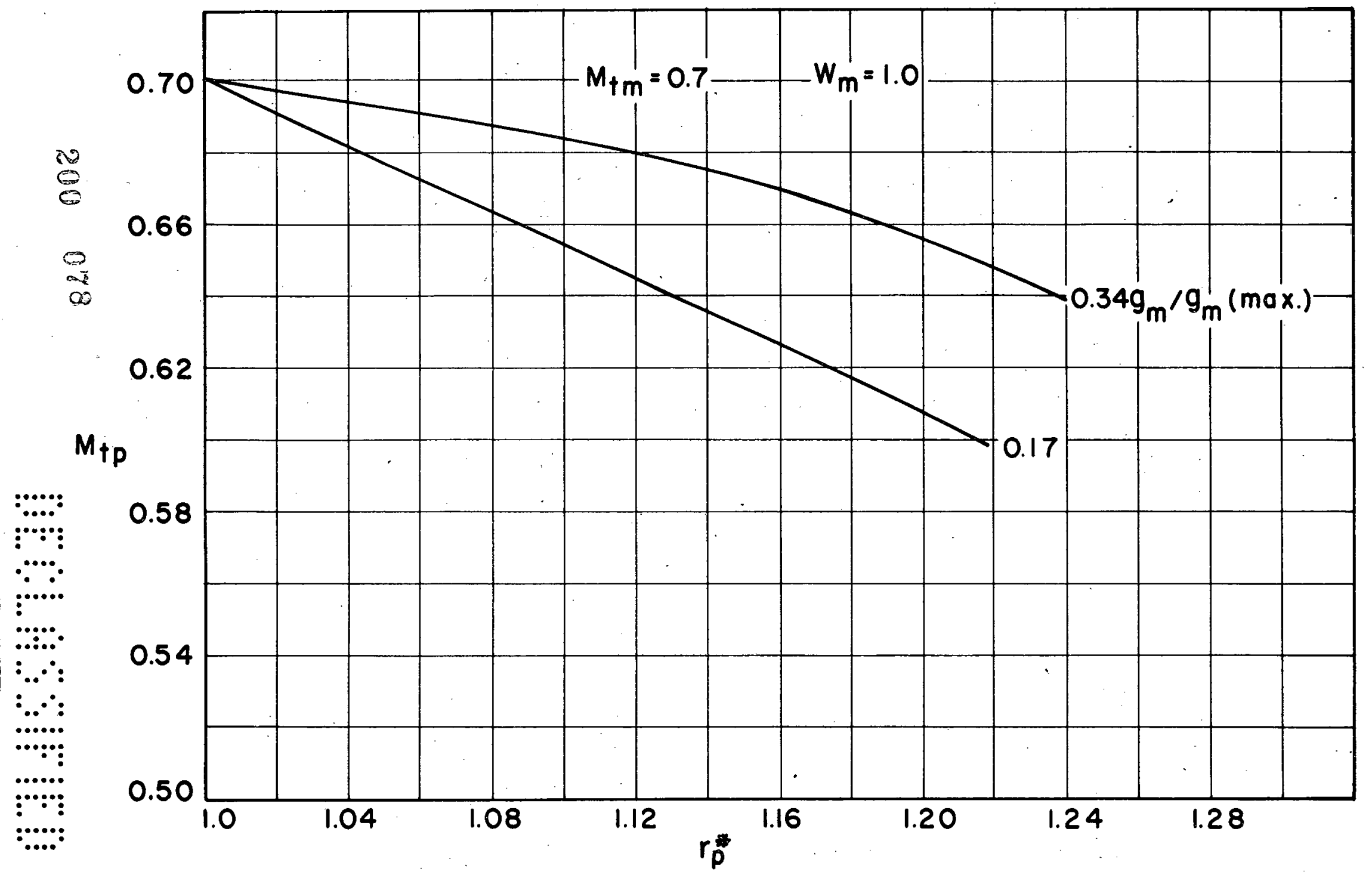

FIG.10a-DEPENDENCE OF THE TANGENTIAL MACH NUMBER AT THE PERIPHERY, M†P, ON THE CHOICE OF THE VALUE, $r_{\bar{p}}^{*}$, OF THE DIMENSIONLESS RADIUS WHICH CORRESPONDS TO THE TUBE PERIPHERY:FOR $g_{m}$ LESS, THAN $g_{m}$ (crit.) 
$\therefore: \cdots:$

$\vdots \ldots: .:$

.....:

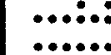

$\because \because$

$\because \because$

$\because \cdots$

$\vdots \ldots$

$\because \because$

$\therefore$

$\therefore \ldots:$

(3)

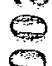

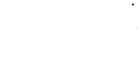

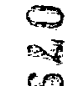

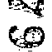

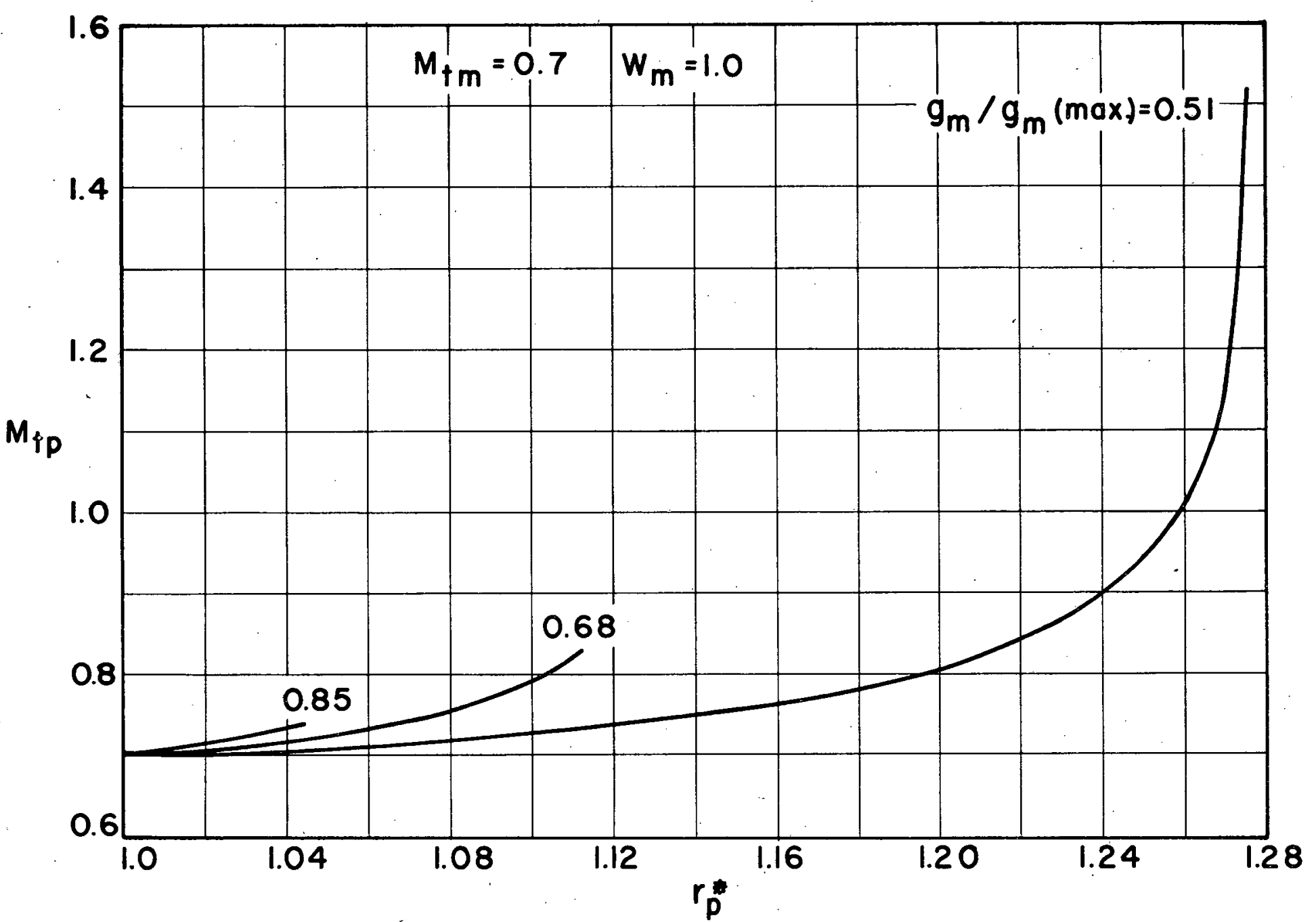

FIG. IOb-DEPENDENCE OF THE TANGENTIAL MACH NUMBER AT THE PERIPHERY, $M_{\uparrow p}, O N$ THE CHOICE OF THE VALUE, $r_{p}^{*}$, OF THE DIMENSIONLESS RADIUS WHICH CORRESPONDS TO THE TUBE PERIPHERY: FOR $g_{m}$ GREATER THAN $g_{m}$ (CRIT) 


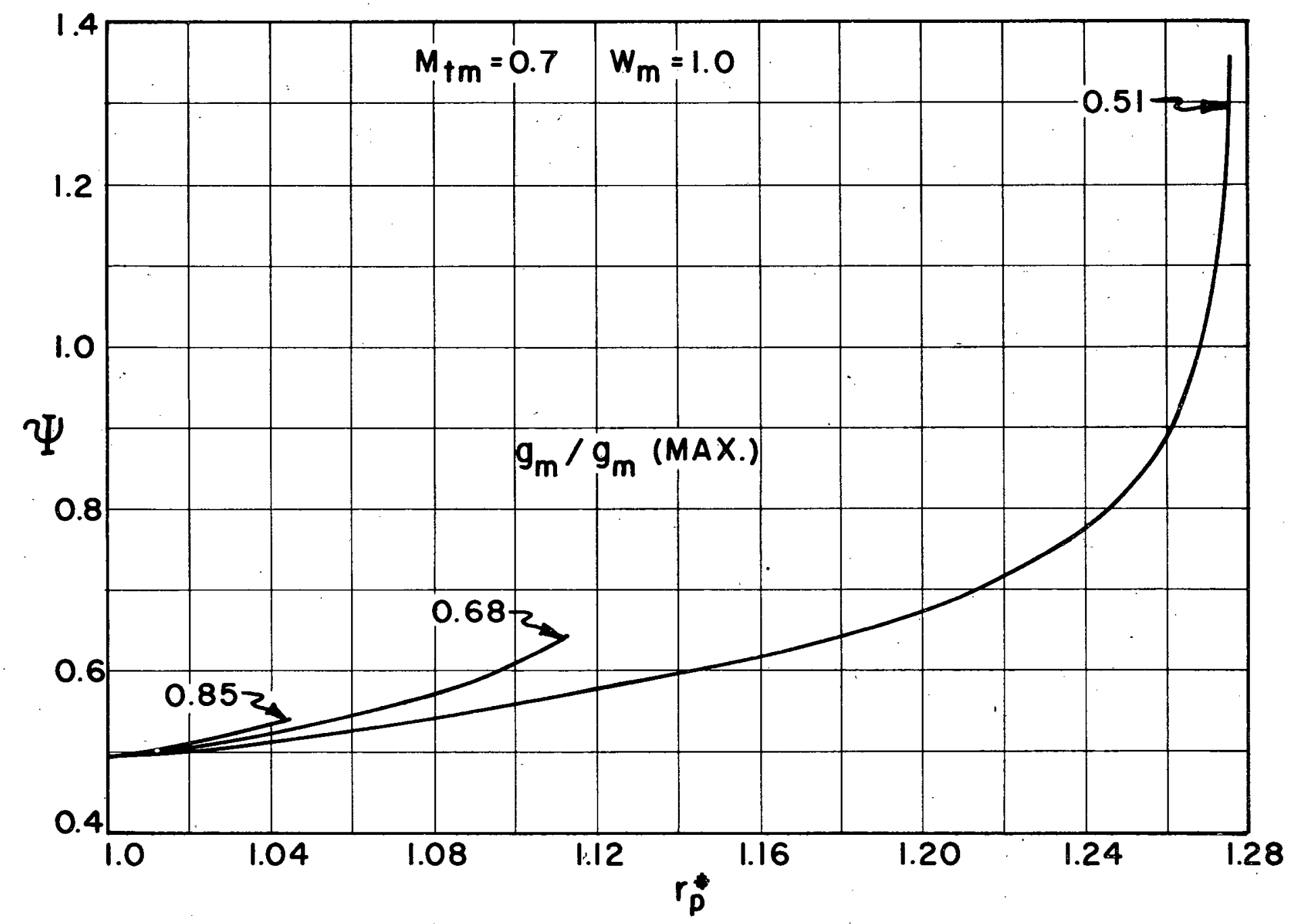

FIG. IIb-DEPENDENCE OF THE RELATIVE MASS FLOW CAPACITY, $\Psi, O N$ THE CHOICE OF THE VALUE, $r_{p}^{*}$, OF THE DIMENSIONLESS RADIUS WHICH CORRESPONDS TO THE TUBE PERIPHERY: FOR $g_{m}$ GREATER THAN $g_{m}$ (CRIT.) 


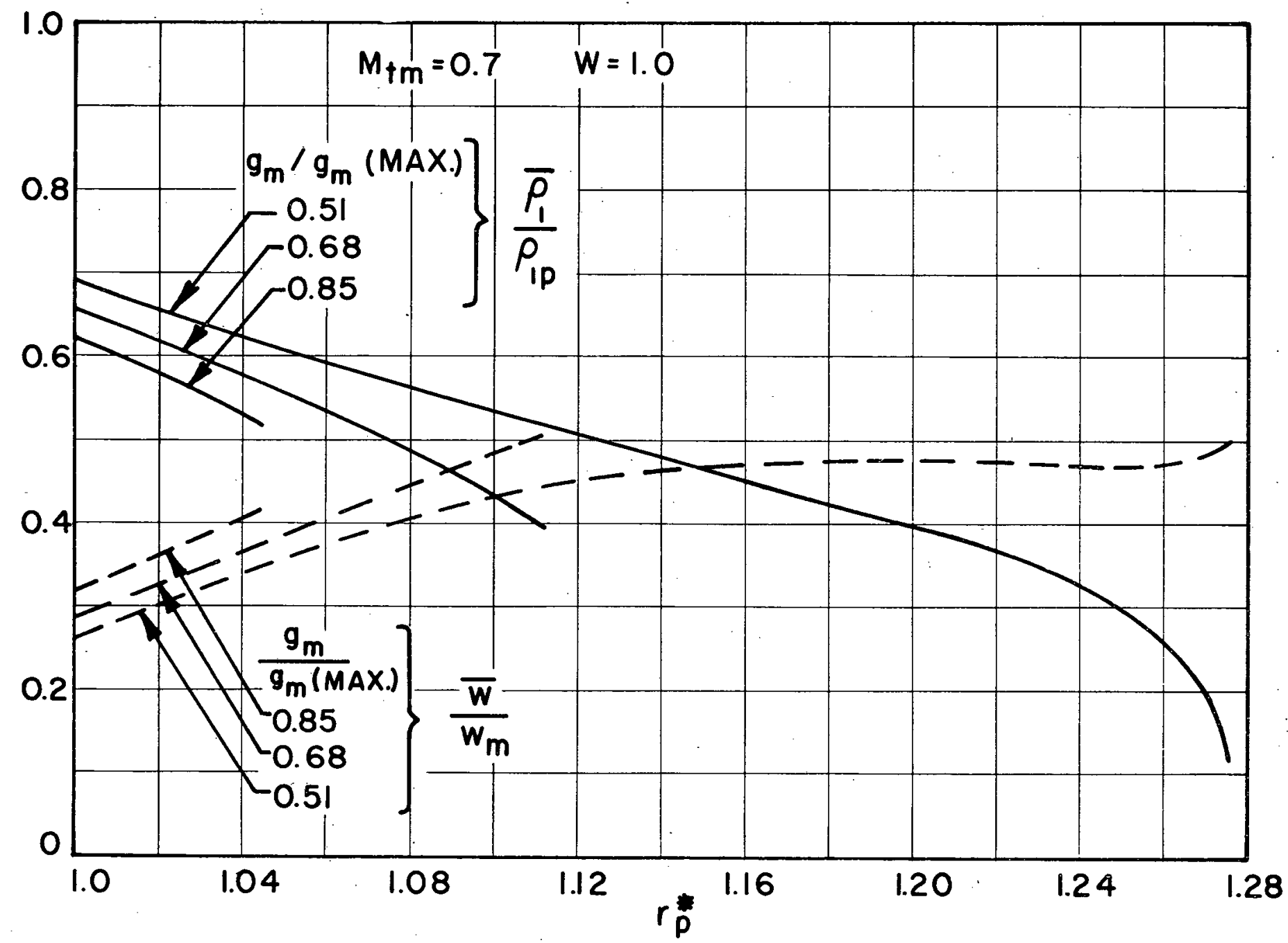

FIG.I2b-DEPENDENCE OF THE AVERAGE-TO-PERIPHERAL PROPELLANT DENSITY RATIO, AND THE RATIO OF AVERAGE TO MAXIMUM DENSITY RATIO ON THE CHOICE OF THE VALUE, $r_{p}^{*}$, OF THE DIMENSIONLESS RADIUS WHICH CORRESPONDS TO THE TUBE PERIPHERY: FOR $g_{m}$ GREATER THAN $g_{m}$ (CRIT.) 


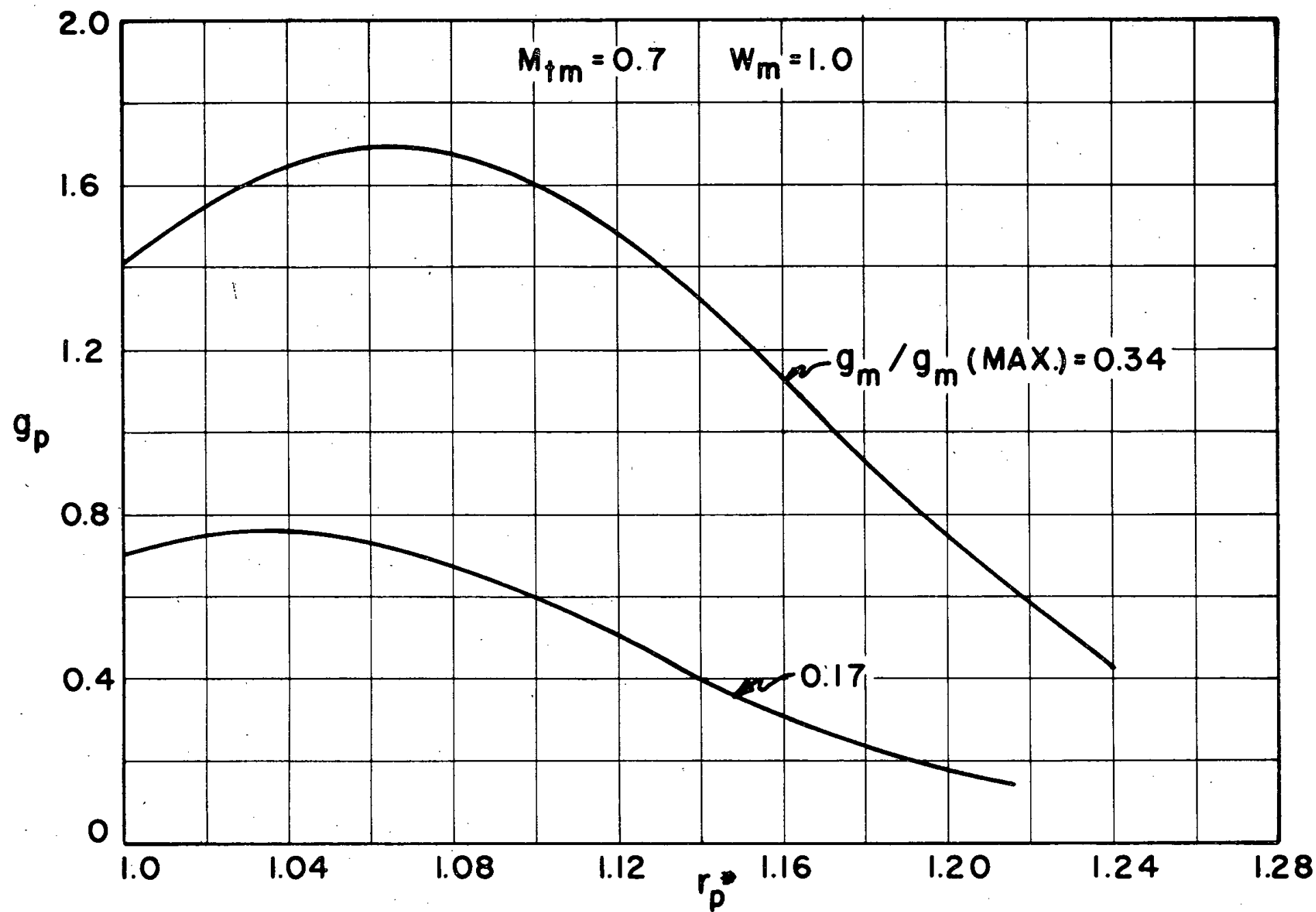

FIG. 130 -DEPENDENCE of THE heATING PARAMETER, $g_{p}$, on the VAlue, $r_{p} ;$ of the DIMENSIONLESS RADIUS WHICH CORRESPONDS TO THE TUBE PERIPHERY: FOR $g_{m}$ LESS THAN $g_{m}$ (CRIT.) 


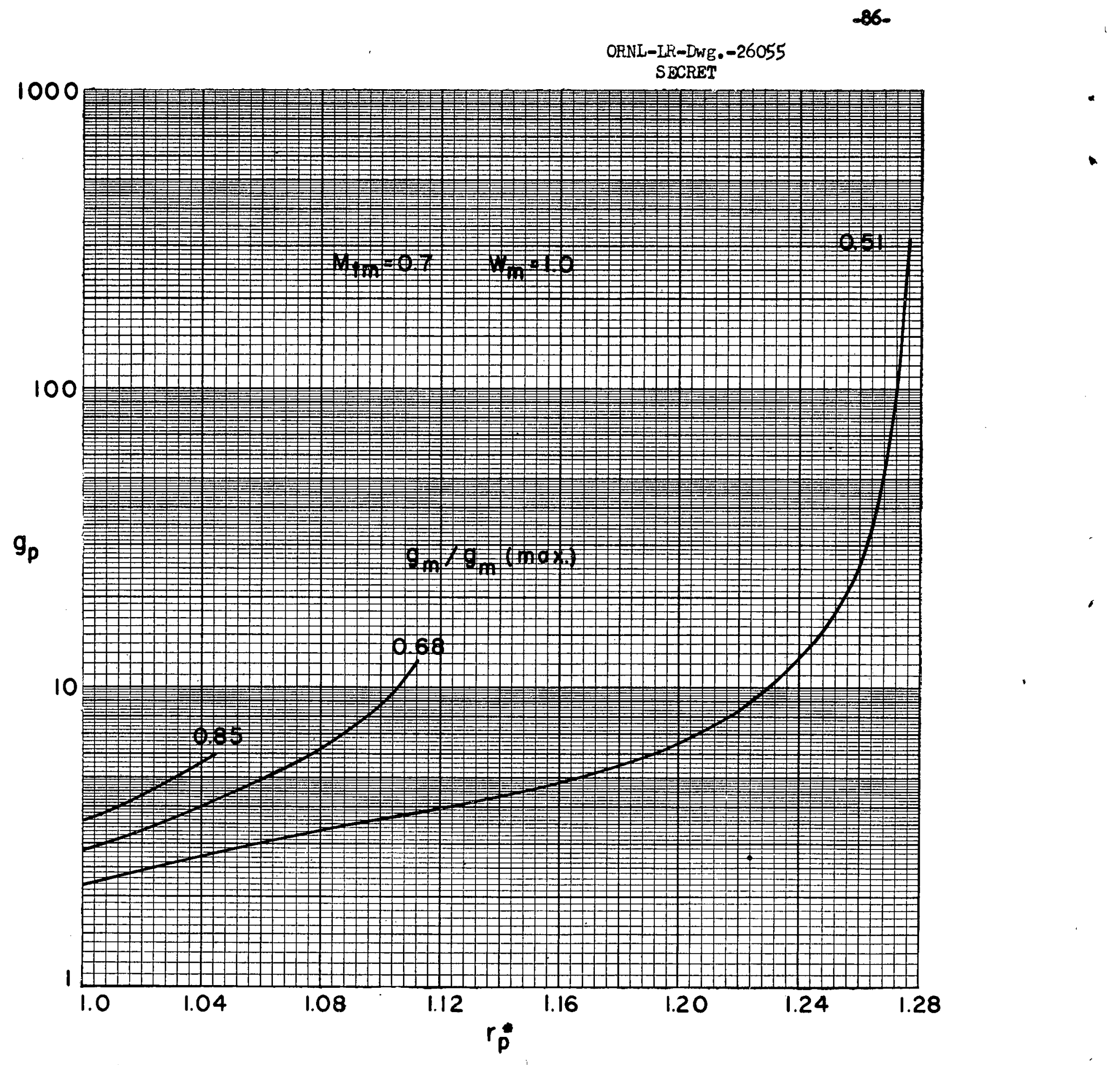

FIG.13b-DEPENDENCE OF THE HEATING PARAMETER, $g_{p}$, ON THE VALUE, $r_{p}^{*}$, OF THE DIMENSIONLESS RADIUS WHICH CORRESPONDS TO THE TUBE PERIPHERY: FOR $g_{m}$ GREATER THAN $g_{m}$ (CRIT.)

$$
\text { : }
$$




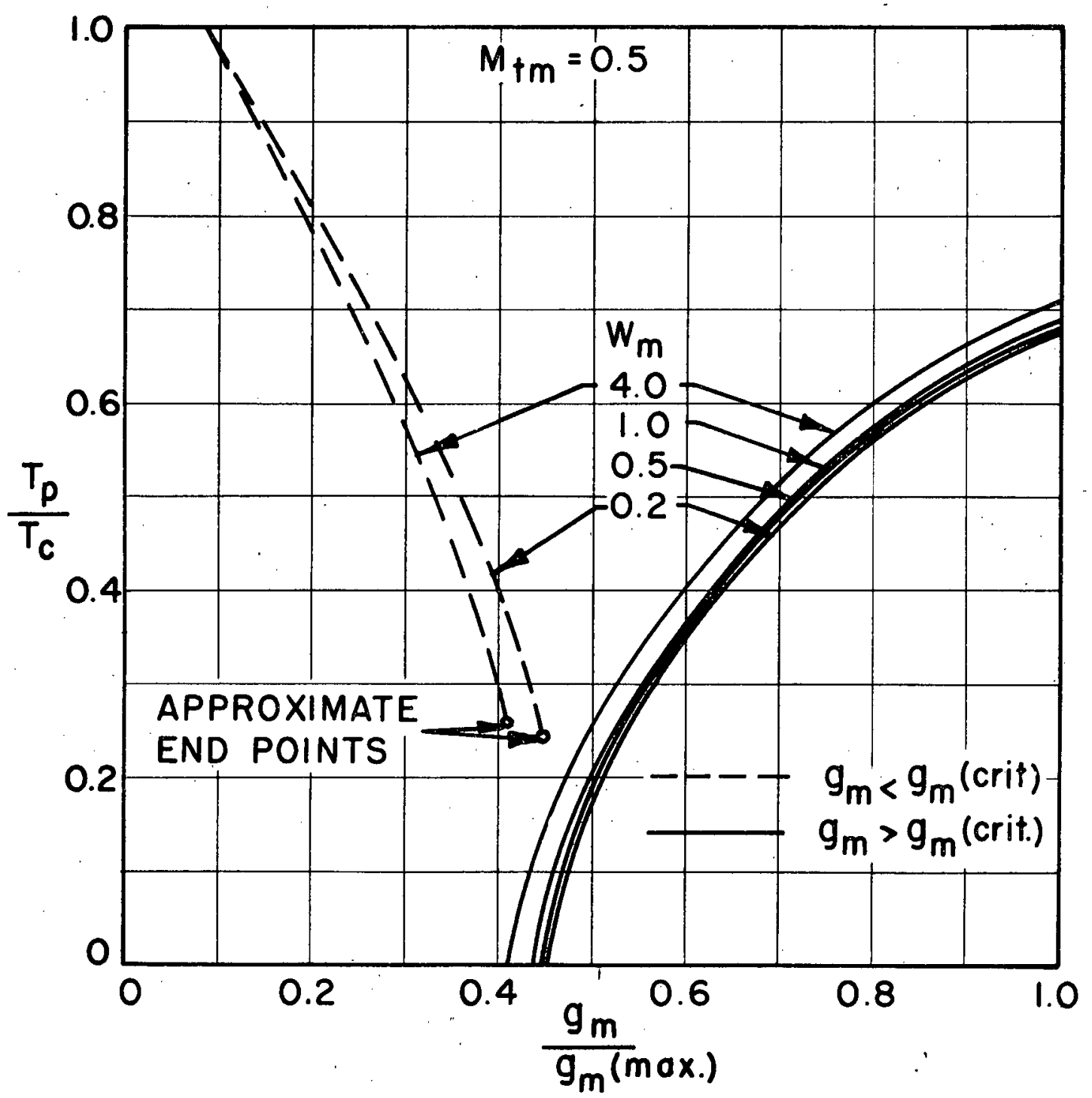

FIG.140-DEPENDENCE OF THE OVERALL TEMPERATURE RATIO, $T_{p} / T_{c}$, ON THE RATIO OF THE HEATING PARAMETER $g_{m}$, TO ITS MAXIMUM PERMISSIBLE VALUE:FOR $M_{+m}=0.5$ 
ORNL-LR-Dwg, -26057

SECRET

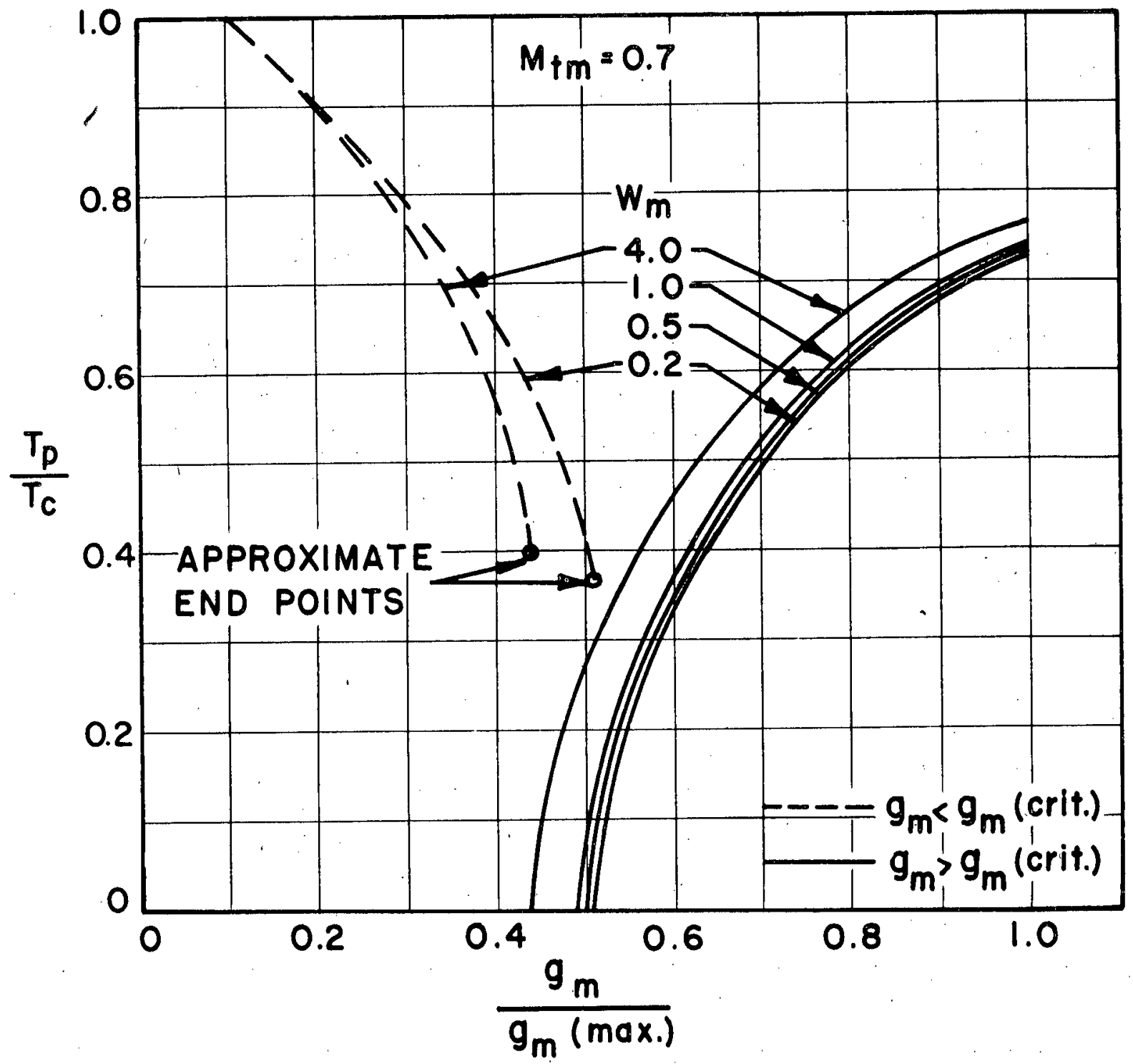

FIG. 14b-DEPENDENCE OF THE OVERALL TEMPERATURE RATIO, $T_{p} / T_{c}, O N$ THE RATIO OF THE HEATING PARAMETER $g_{m}$, TO ITS MAXIMUM PERMISSIBLE VALUE: FOR $M_{t m}=0.7$ 


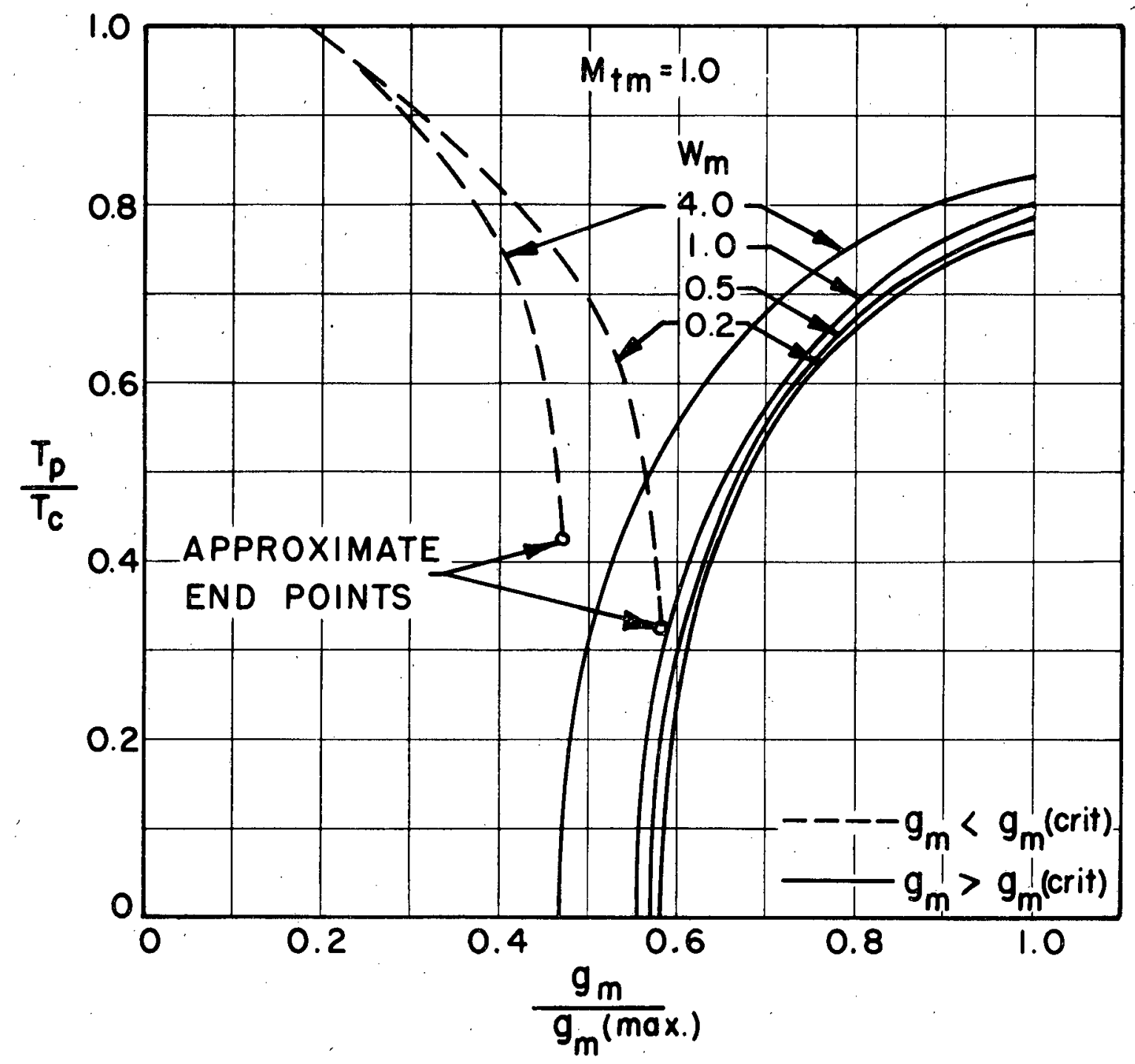

FIG.14C-DEPENDENCE OF THE OVERALL TEMPERATURE RATIO; $T_{p} / T_{c}$, ON THE RATIO OF THE HEATING PARAMETER $g_{m}$, TO ITS MAXIMUM PERMISSIBLE VALUE:FOR $M_{\uparrow m}=1.0$ 


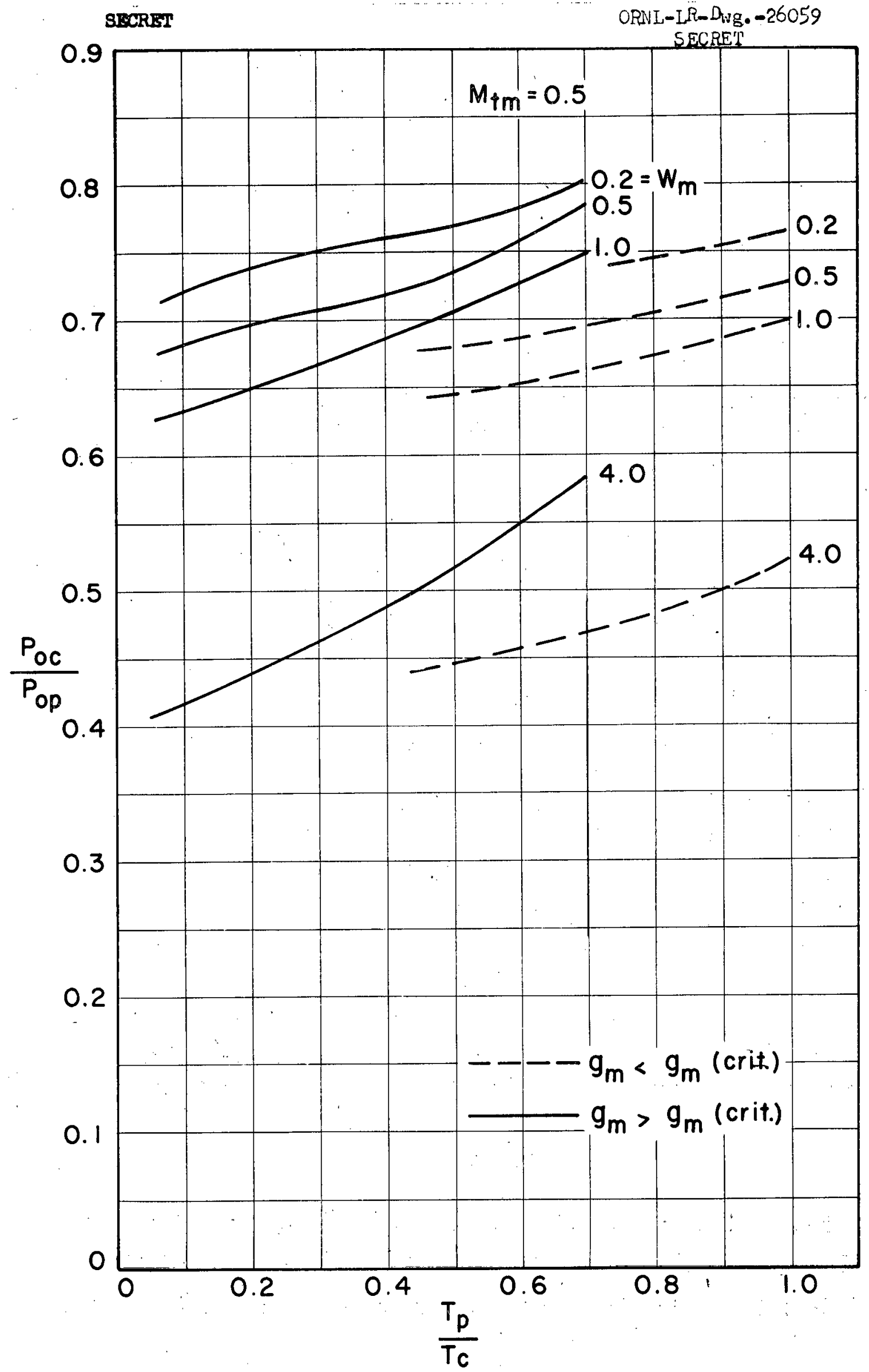

-90 - 


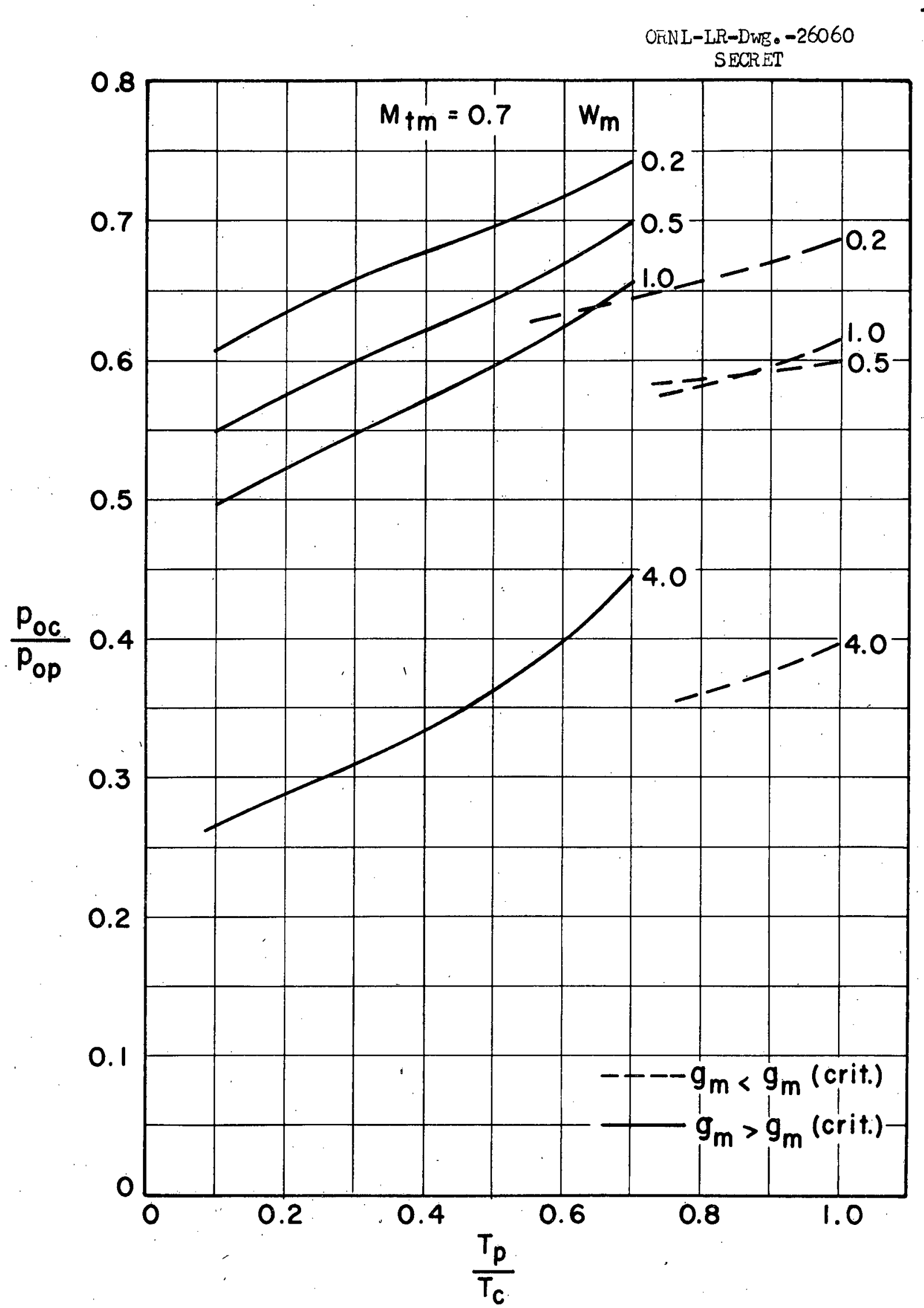

FIG. $15 b$-DEPENDENCE OF THE OVERALL PRESSURE RATIO, $P_{O C} / P_{o p}$, ON THE OVERALL TEMPERATURE RATIO, $T_{p} / T_{c}:$ FOR $M_{+m}=0.7$

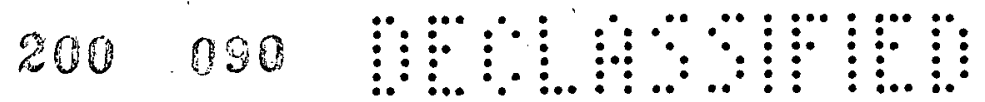




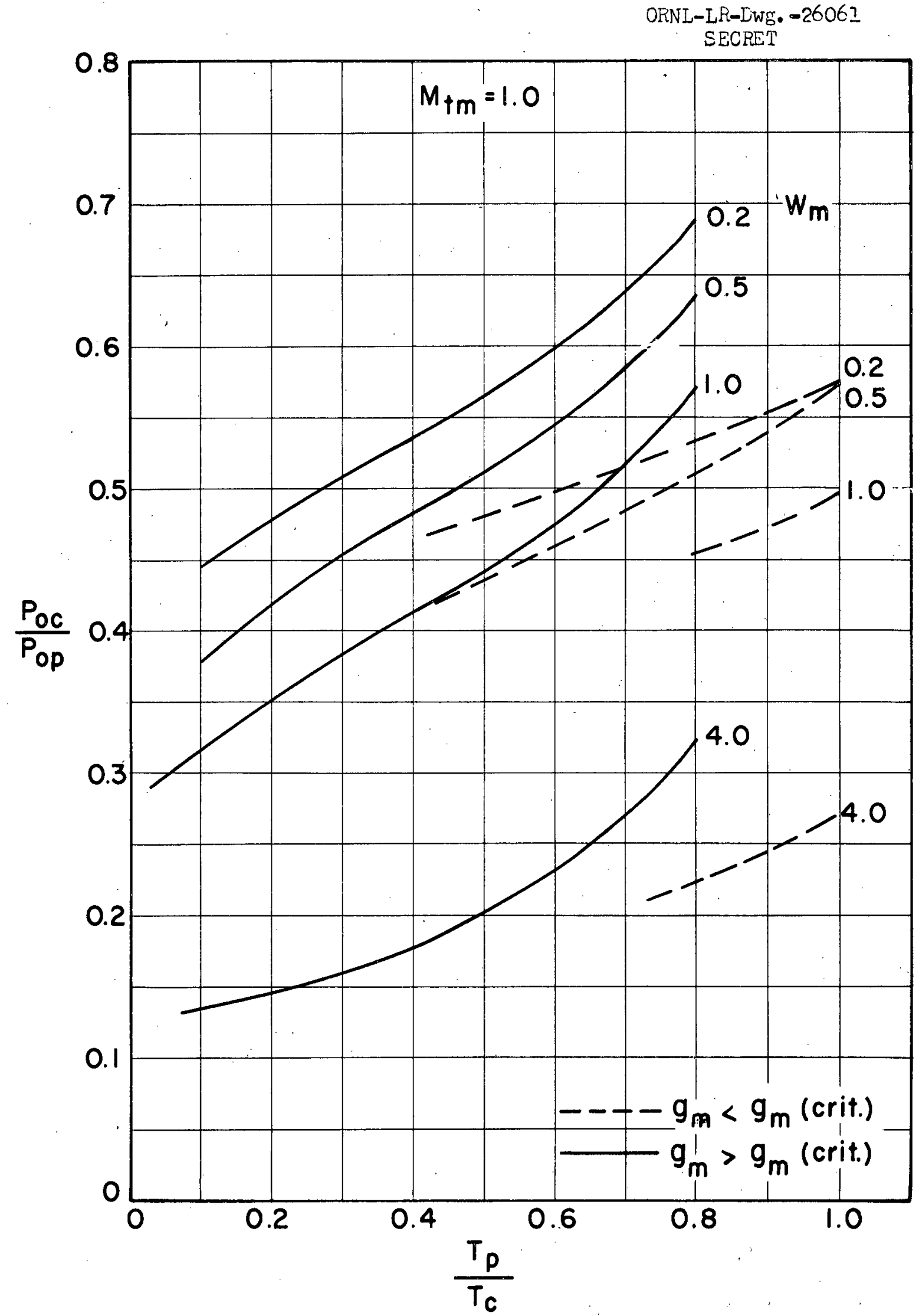

FIG. 15C-DEPENDENCE OF THE OVERALL PRESSURE RATIO, $P_{o c} / P_{o p}$, ON THE OVERALL TEMPERATURE RATIO, $T_{p} / T_{c}: F O R M_{t m}=1.0$

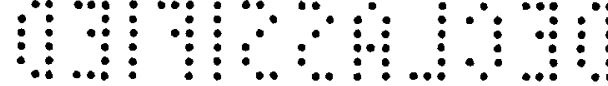
$20003 \mathbb{R}^{2}$ 


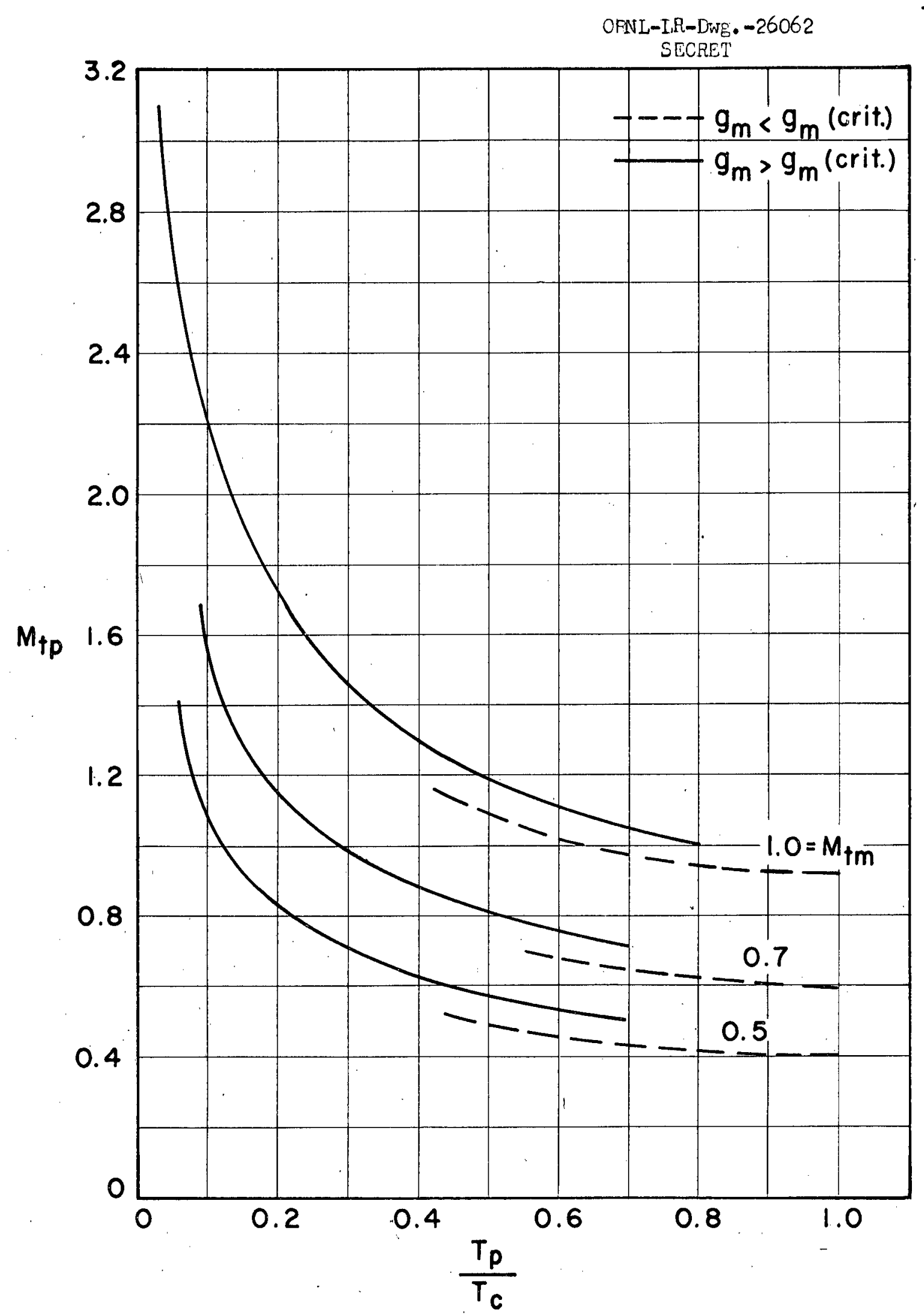

FIG.16-DEPENDENCE OF THE TANGENTIAL MACH NUMBER AT THE PERIPHERY, $M_{t p}$, ON THE OVERALL TEMPERATURE RATIO, $T_{c} / T_{p}$ 
ORNL-IR-Dwg. -26063

SECRET

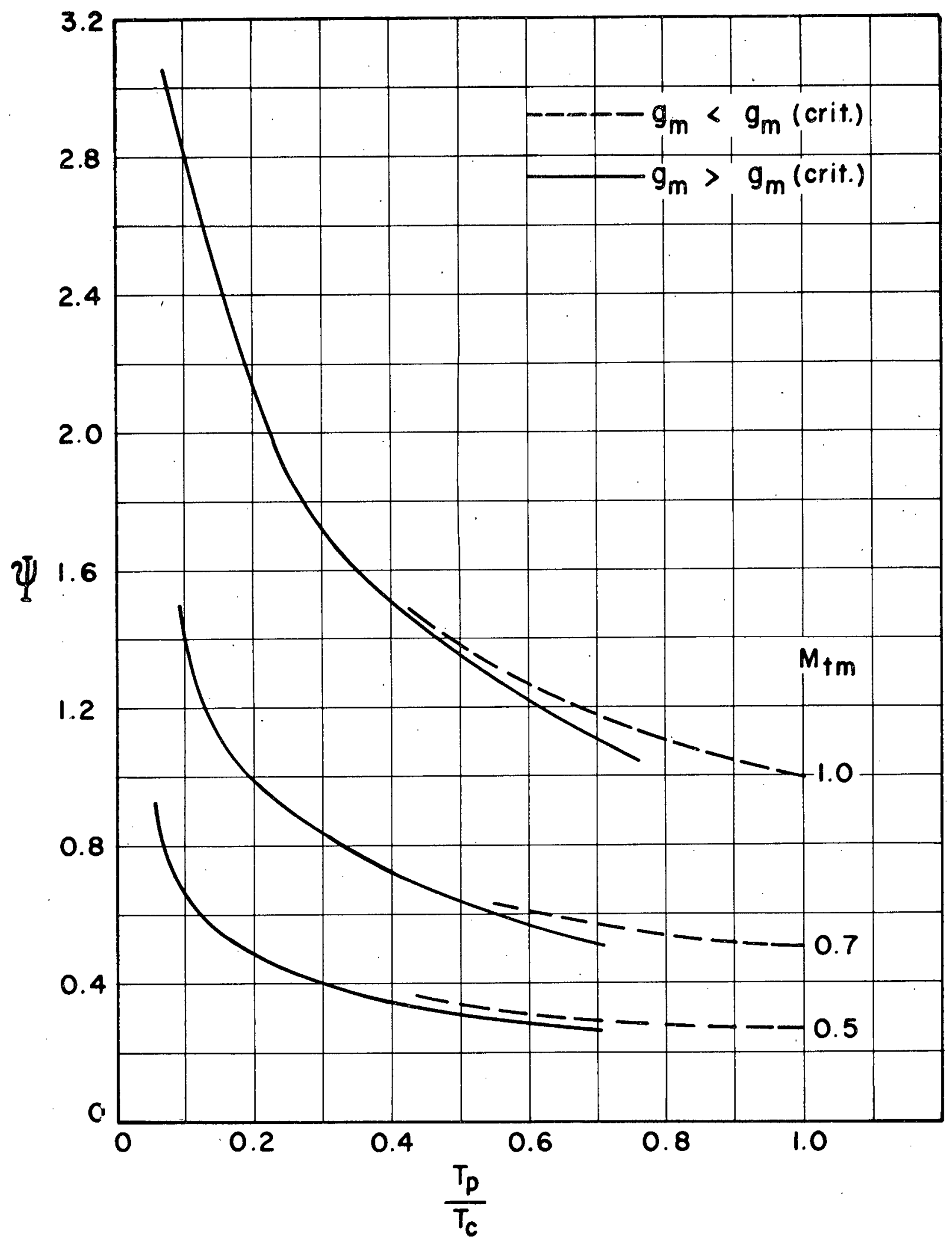

FIG.17-DEPENDENCE OF THE RELATIVE MASS FLOW CAPACITY, $\Psi$, ON THE OVERALL TEMPERATURE RATIO, $T_{p} / T_{2} G 0$ 
ORNL-LR-Dwg -26064 SECRET

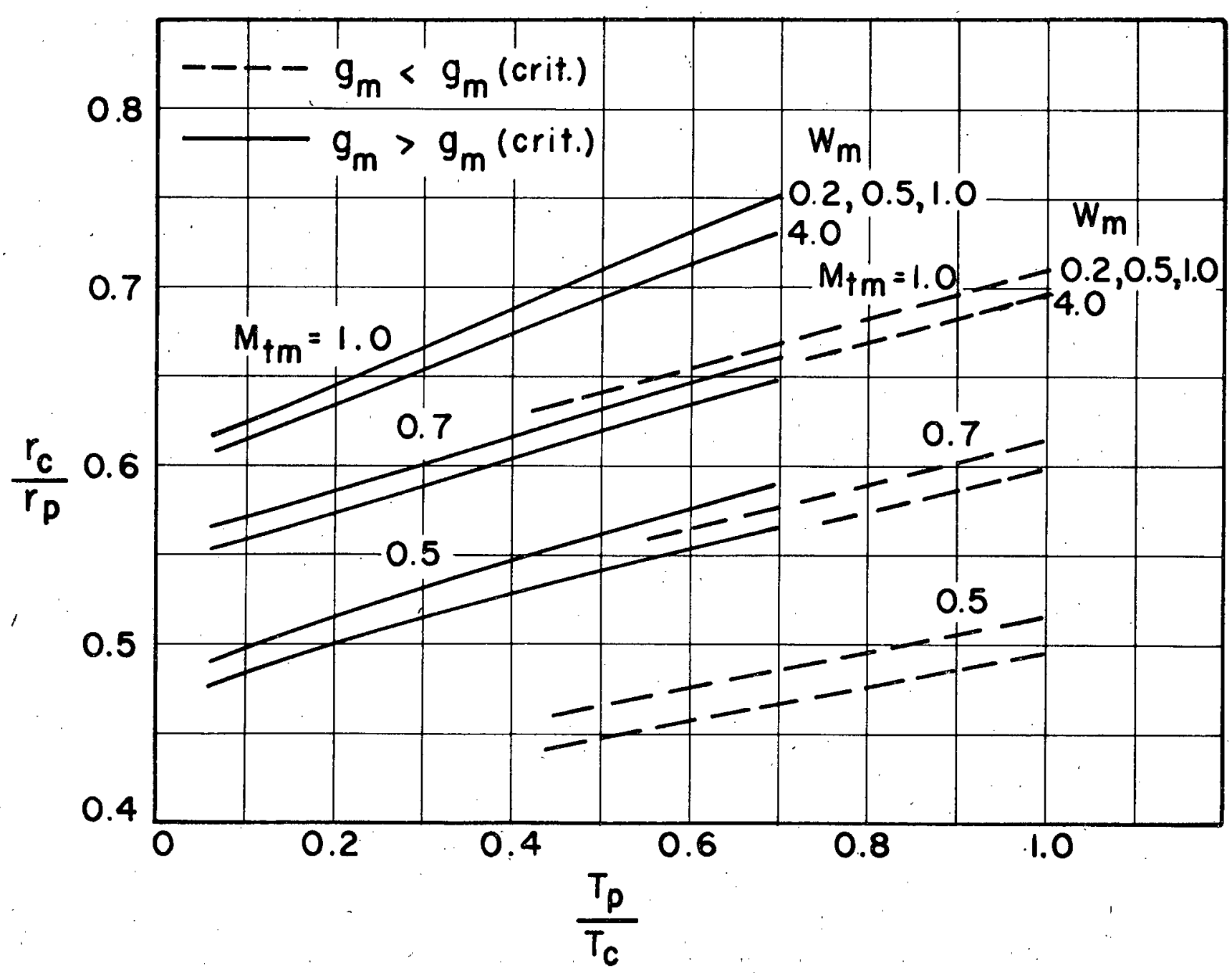

FIG.18-DEPENDENCE OF THE RATIO OF EXIT RADIUS TO TUBE RADIUS ON THE OVERALL TEMPERATURE RATIO $T_{p} / T_{c}$. 


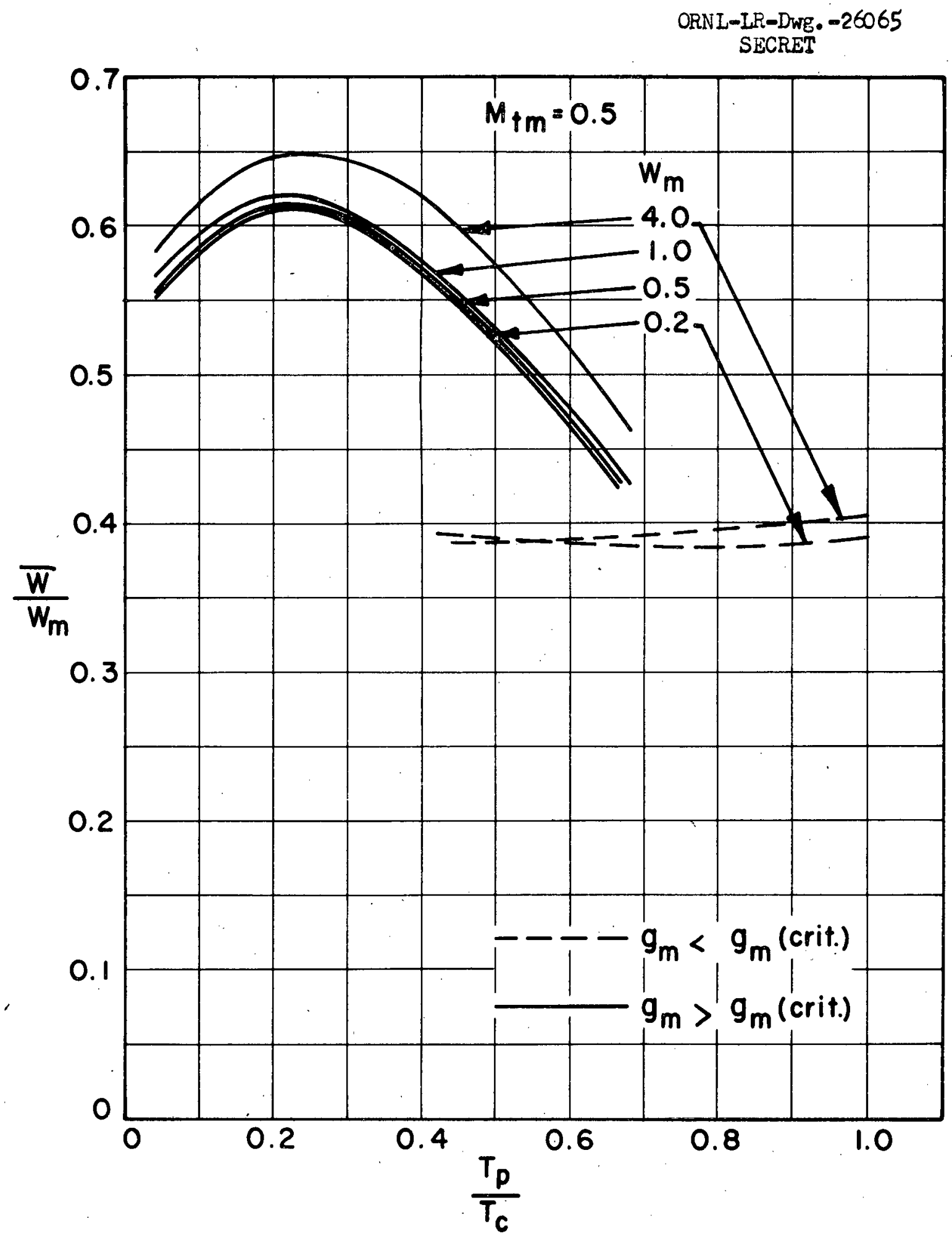

FIG.19a-DEPENDENCE OF THE RATIO OF AVERAGE TO MAXIMUM DENSITY RATIO ON THE OVERALL TEMPERATURE RATIO, $T_{p} / T_{c}$ : FOR $M_{t m}=0.5$ 200.095 


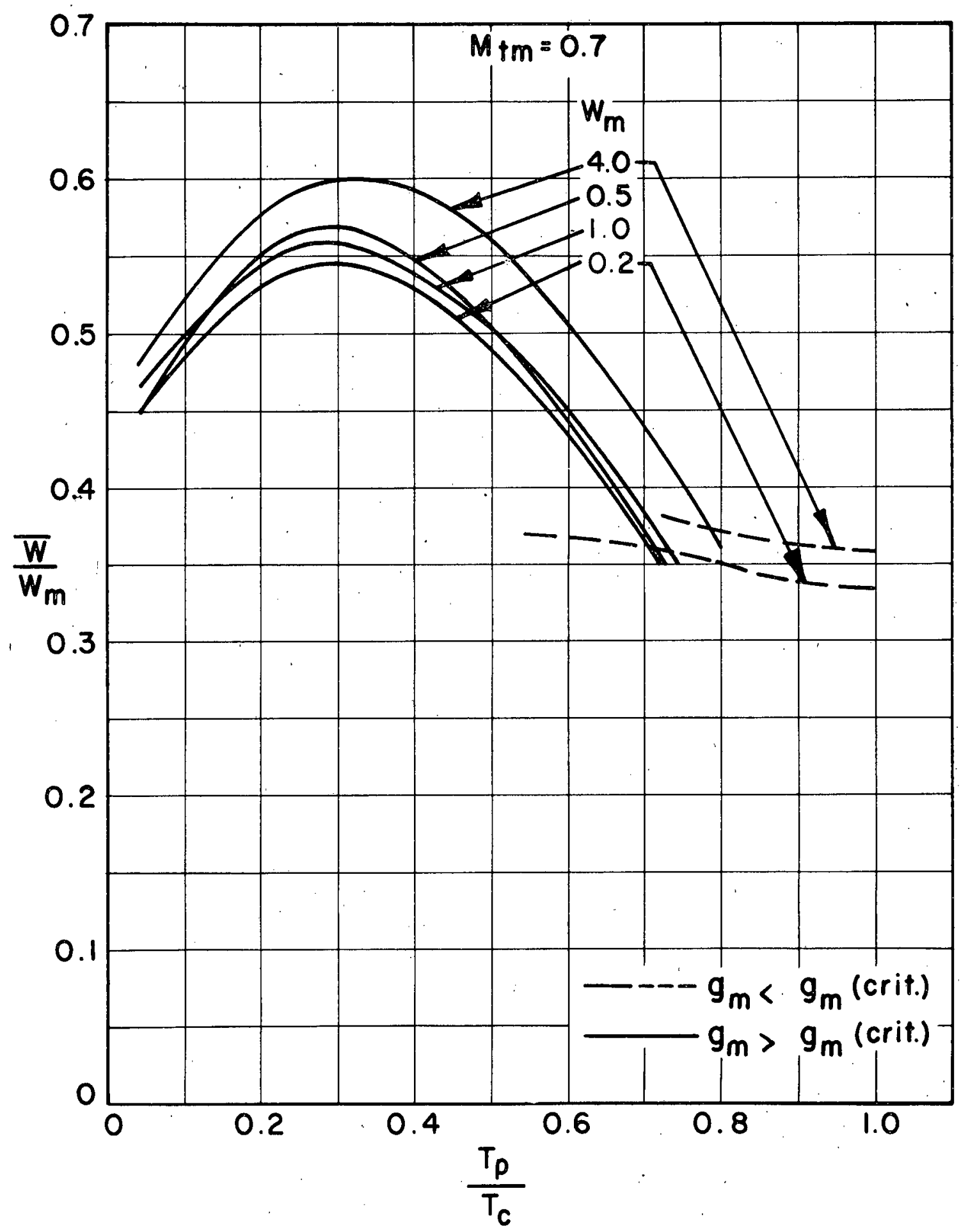

FIG.19b-DEPENDENCE OF THE RATIO OF AVERAGE TO MAXIMUM DENSITY RATIO ON THE OVERALL TEMPERATURE RATIO, $T_{p} / T_{c}: F O R \quad M_{f m}=0.7$. 


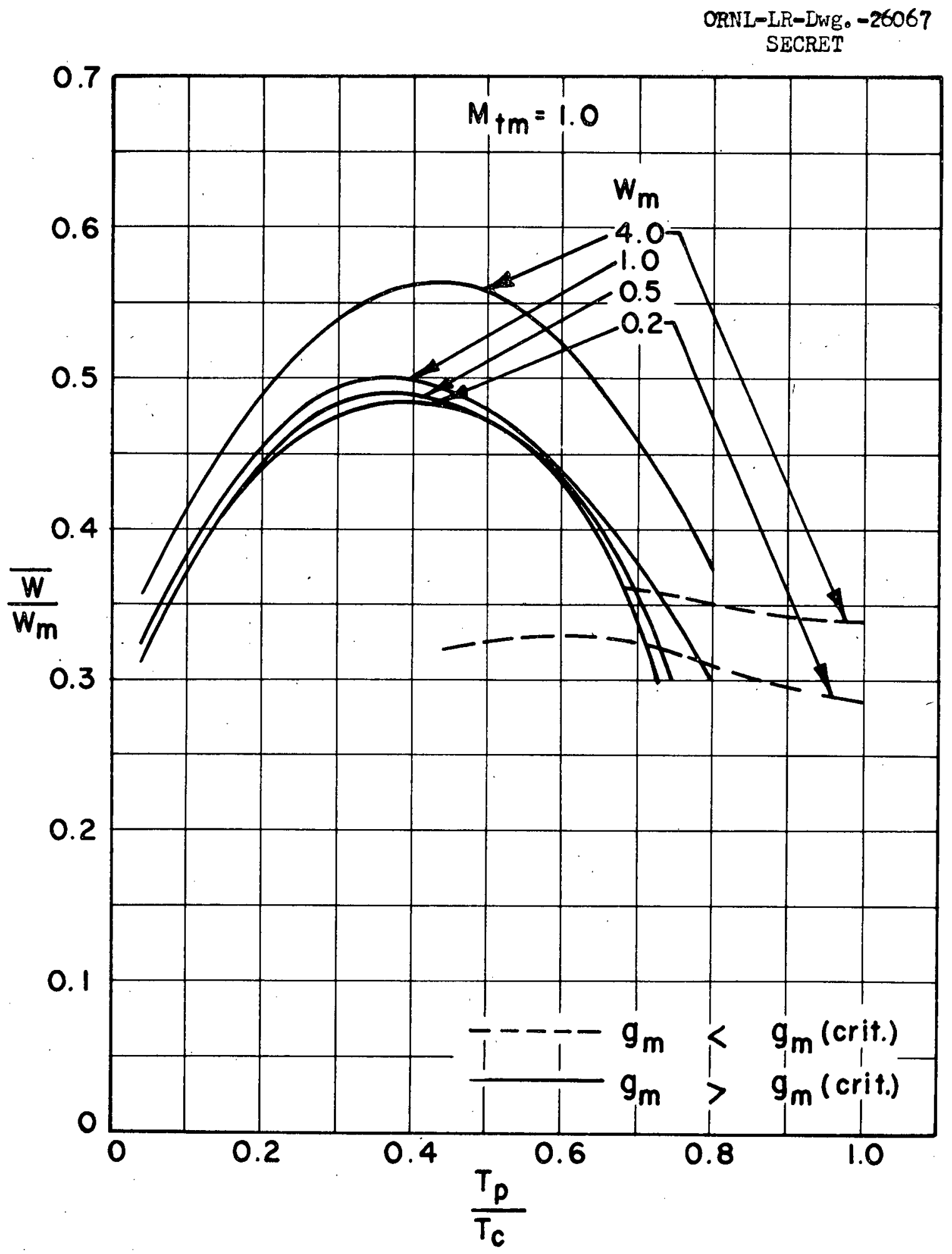

FIG. 19c-DEPENDENCE OF THE RATIO OF AVERAGE TO MAXIMUM DENSITY RATIO ON THE OVERALL TEMPERATURE RATIO, $T_{p} / T_{c}:$ FOR $M_{f m}=1.0$ 


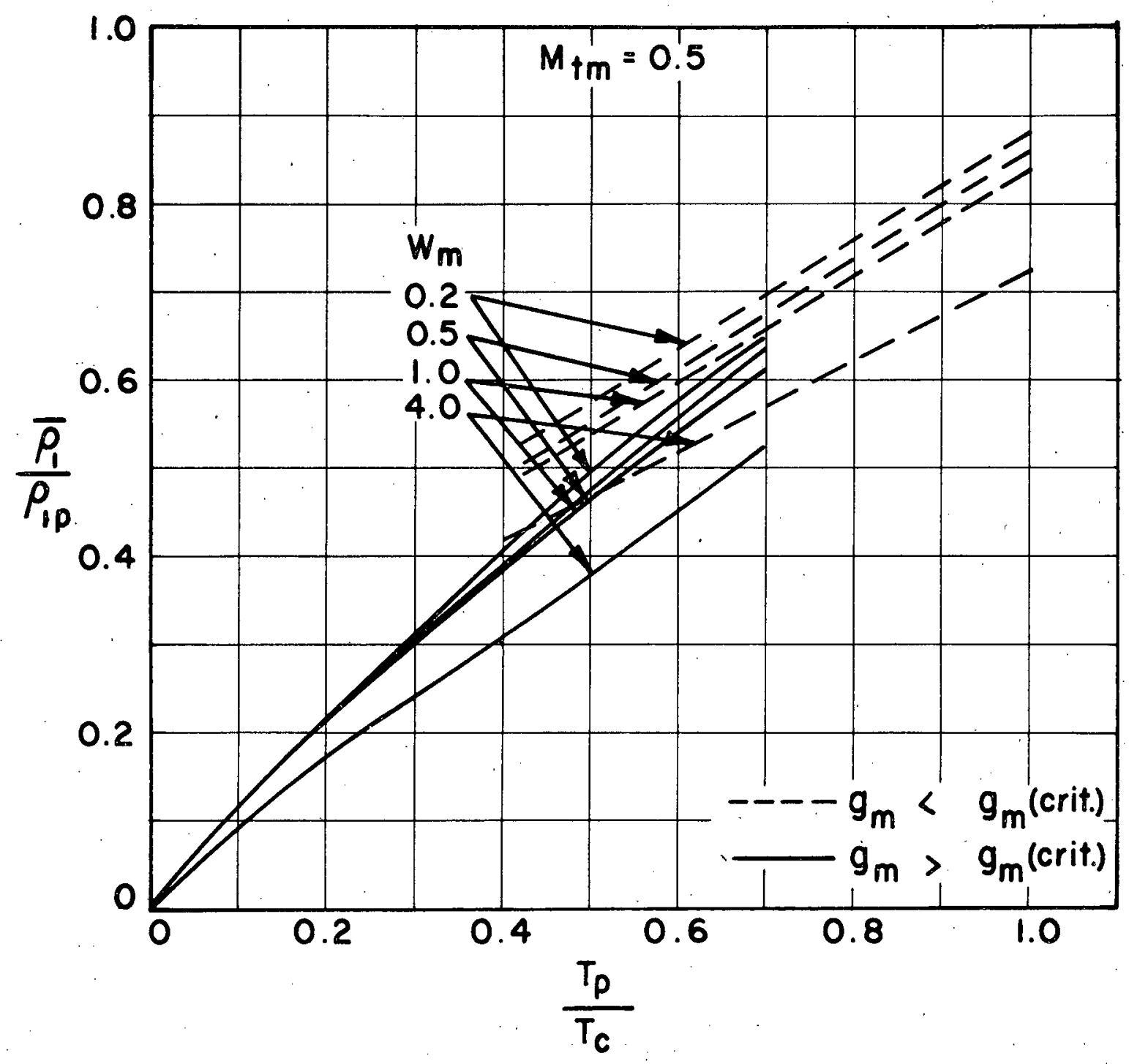

FIG. 20a-DEPENDENCE OF THE RATIO OF AVERAGE TO PERIPHERAL PROPELLANT DENSITIES ON THE OVERALL TEMPERATURE RATIO, $T_{p} / T_{c}:$ FOR $M_{t m}=0.5$ 


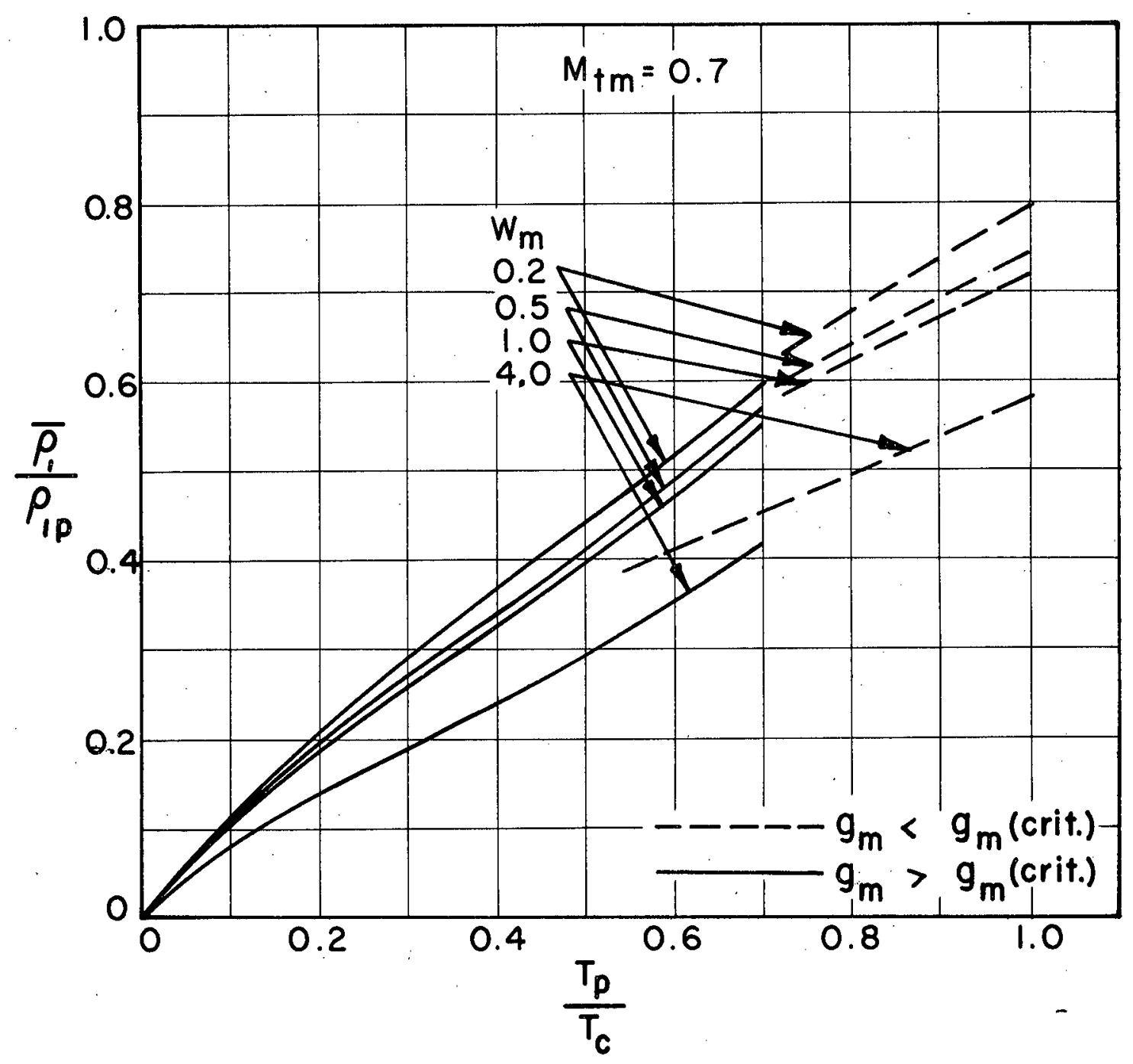

FIG.2Ob-DEPENDENCE OF THE RATIO OF AVERAGE TO PERIPHERAL PROPELLANT DENSITIES ON THE OVERALL TEMPERATURE RATIO, $T_{p} / T_{c}$ : FOR $M_{t m}=0.7$ 
ORNL-LP-DW: - -26070 SECPET

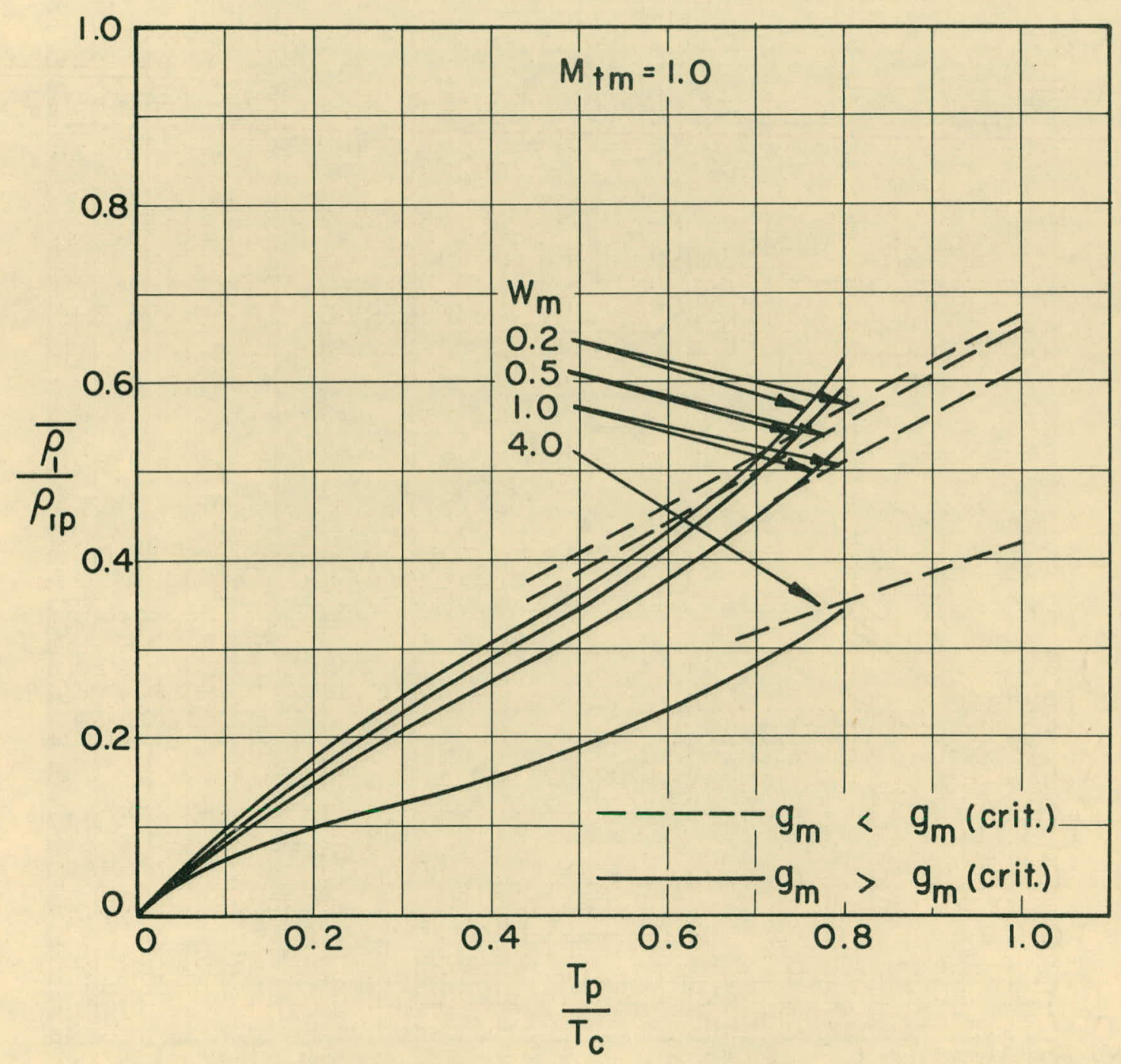

FIG.20C-DEPENDENCE OF THE RATIO OF AVERAGE TO PERIPHERAL PROPELLANT DENSITIES ON THE OVERALL TEMPERATURE RATIO, $T_{p} / T_{c}:$ FOR $M_{t m}=1.0$ 


$$
\begin{gathered}
\text { ORNL-IR-DWg } \\
\text { SECRET }
\end{gathered}
$$

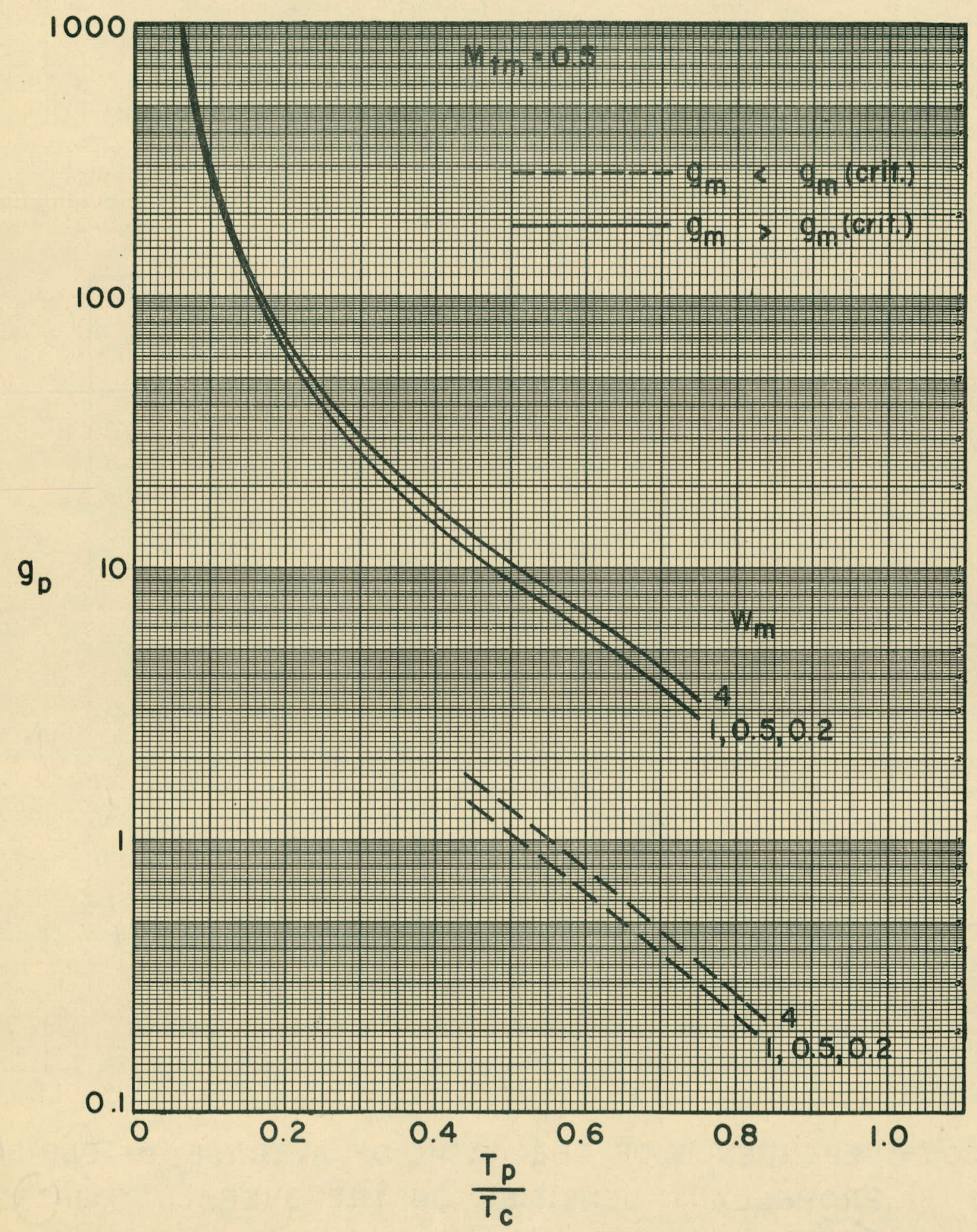

FIG. 2la-DEPENDENCE OF THE HEATING PARAMETER, $g_{p}$, ON THE OVERALL TEMPERATURE RATIO, $T_{p} / T_{c}: F O R \quad M_{+m}=0.5$

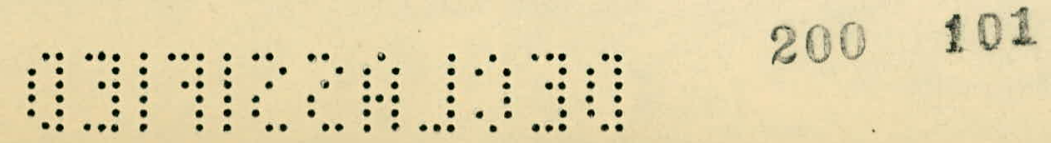




$$
\begin{gathered}
\text { ORNL-LR-Dwg } \\
\text { SECRET }
\end{gathered}
$$

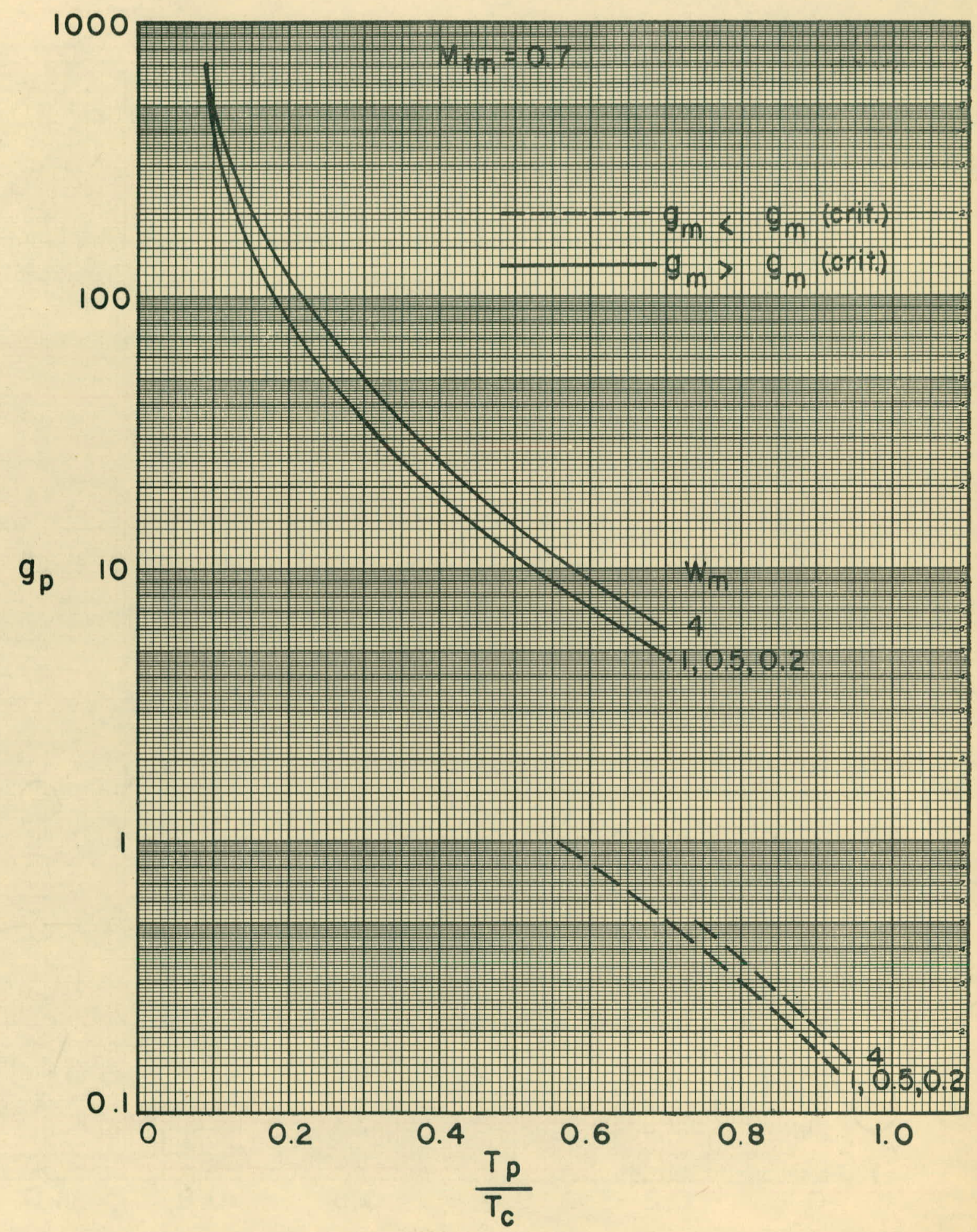

FIG. $21 b$-DEPENDENCE OF THE HEATING PARAMETER, $g_{p}$, ON THE OVERALL TEMPERATURE RATIO, $T_{p} / T_{c}: F O R M_{+m}=0.7$

$$
200102
$$

(艹: 


$$
\underset{\text { SHCRET }}{\text { ORNL-LR-Dwg, }}-26073
$$

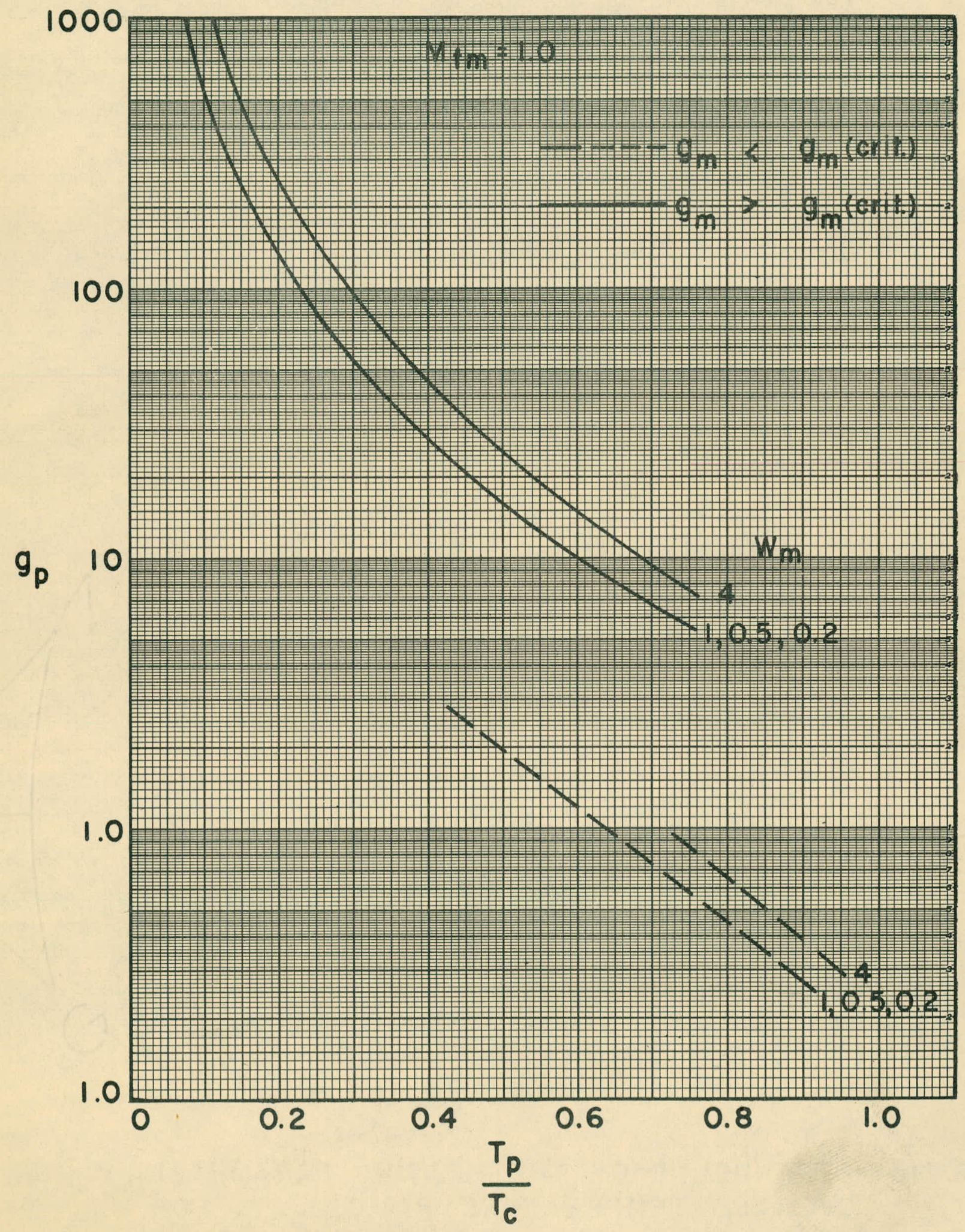

FIG. 2 Ic-DEPENDENCE OF THE HEATING PARAMETER, $g_{p}, O N$ THE OVERALL TEMPERATURE RATIO, $T_{p} / T_{c}: F O R \quad M_{t m}=1.0$

$\because \because: \because: \because \because \because: \because \because \because \because: \because \quad 200 \quad 100$

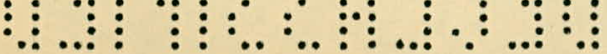


ORNL-IR-DWg. -26074 SECRET

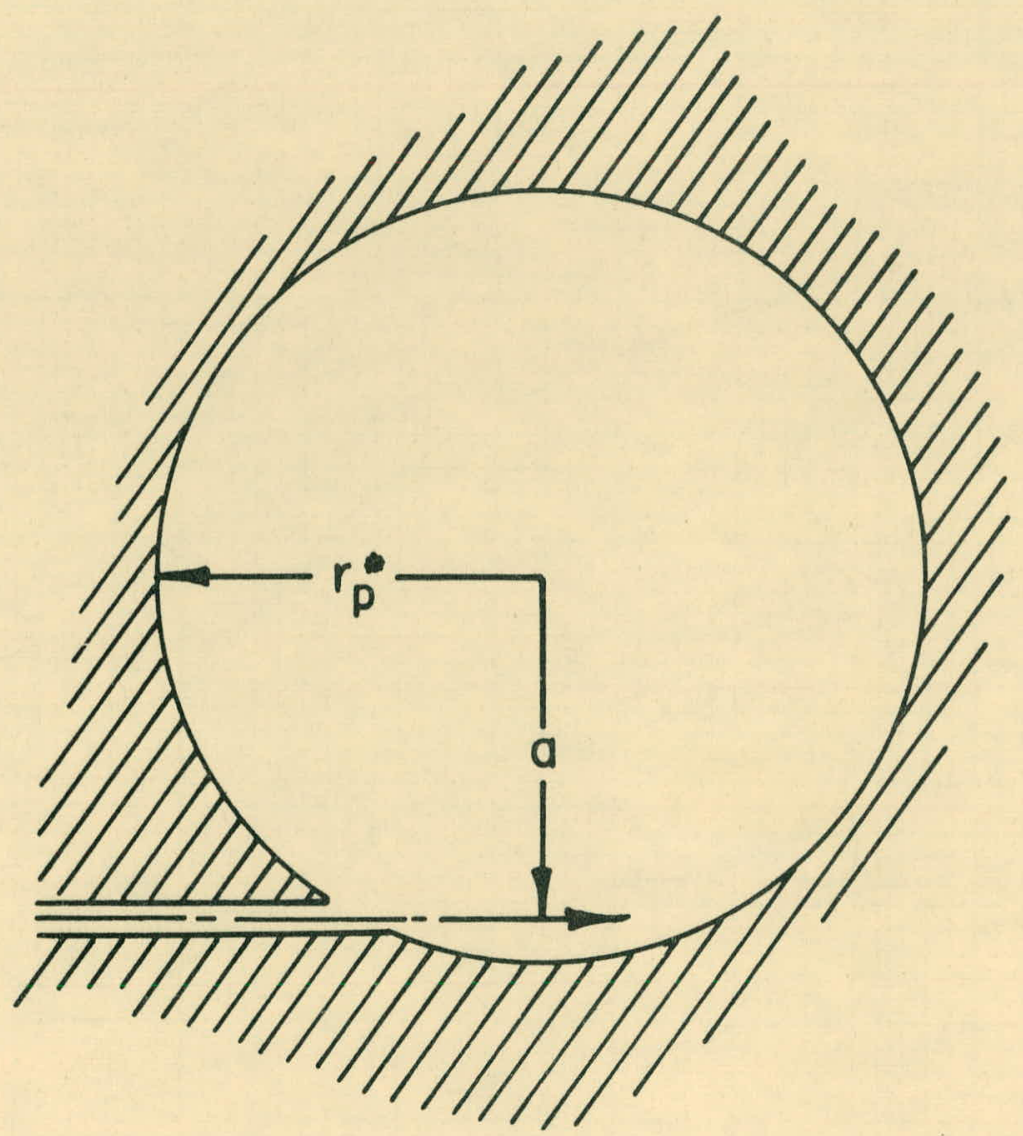

FIG. 22 - INTRODUCTION OF THE FLUID AT A DIMENSIONLESS RADIUS $a$. 


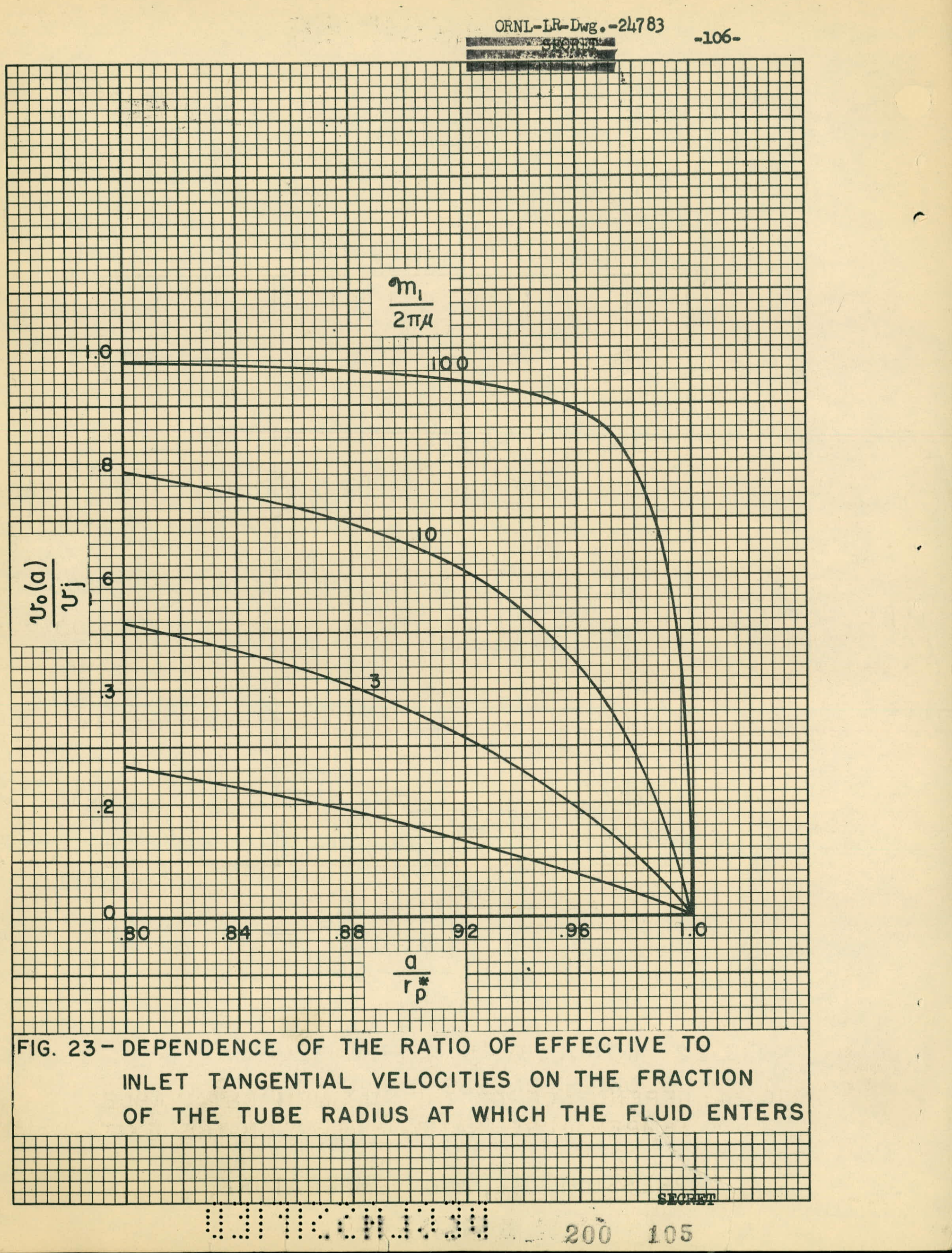




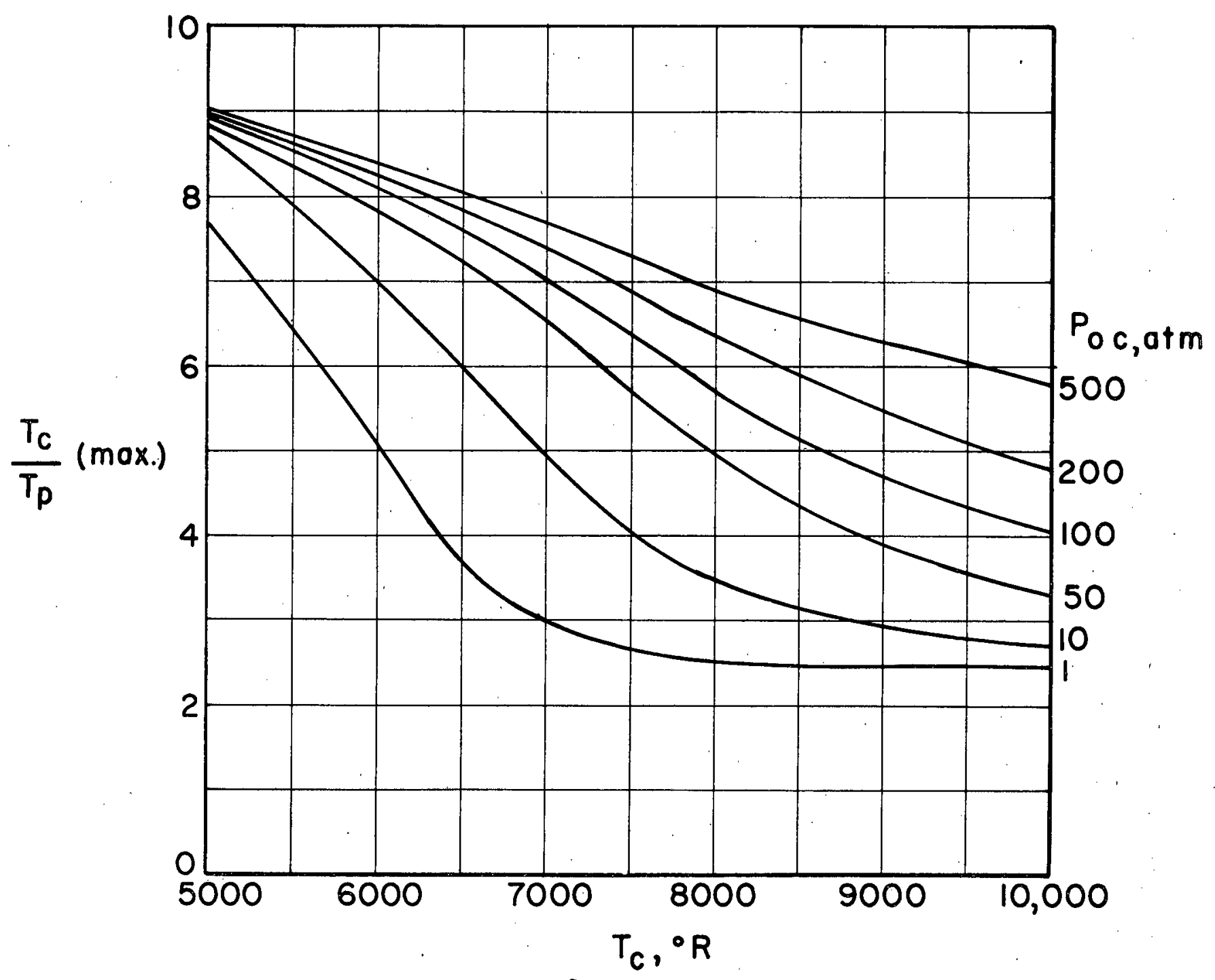

FIG. 24-DEPENDENCE OF THE MAXIMUM OBTAINABLE TEMPERATURE RATIO, $\underline{T}_{c}$ (max.), ON THE EXIT $T_{p}$ 200 106

PRESSURE, PoG: AND TEMPERATURE, TC 


\section{UNGLASSIFIED}
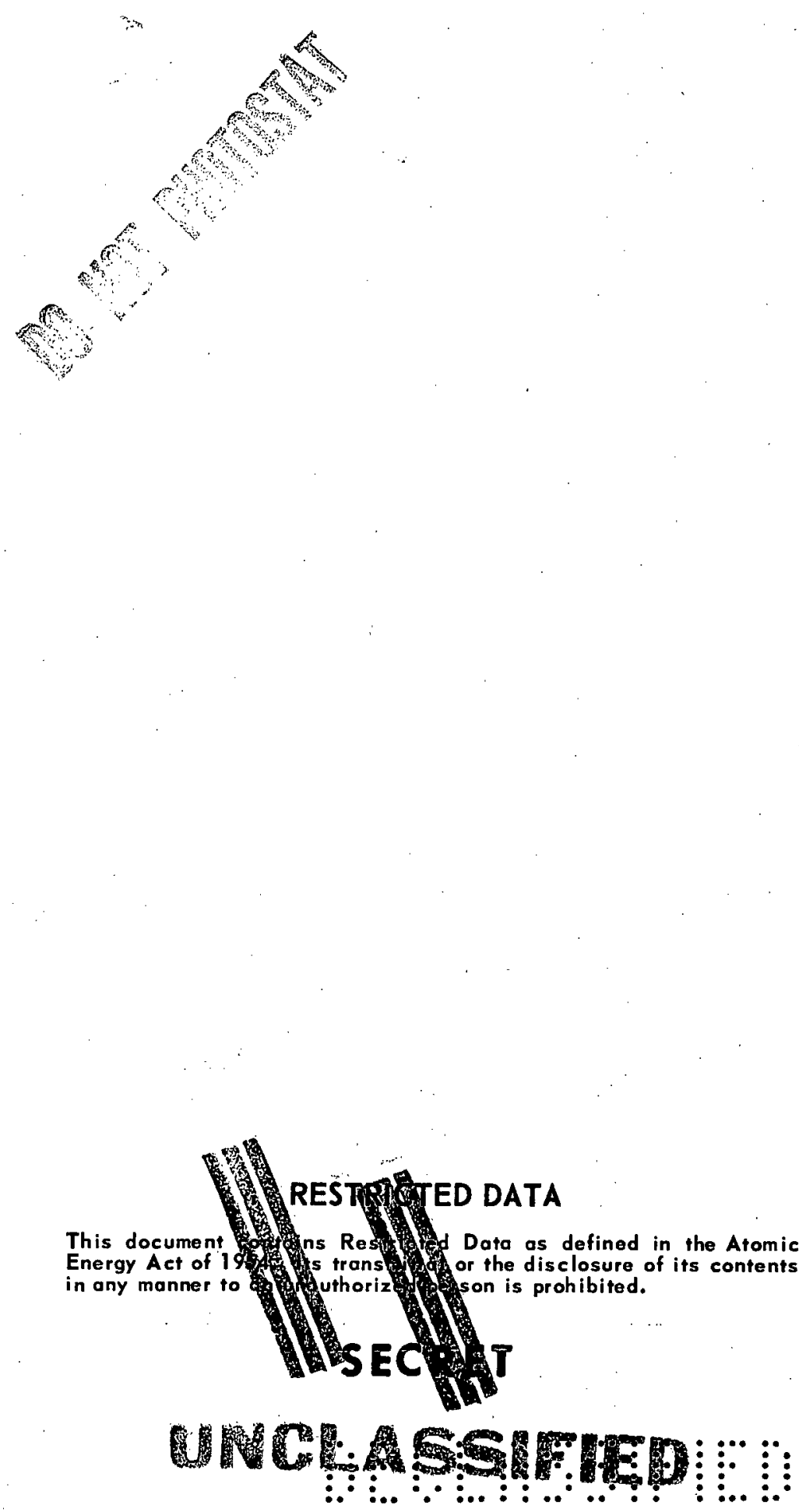